\title{
Radiationless relaxation and optical dephasing of molecules excited by wide- and narrow-band lasers. II. Pentacene in low-temperature mixed crystals
}

\author{
T. E. Orlowski ${ }^{\text {al }}$ and A. H. Zewail ${ }^{\text {bl }}$ \\ Arthur Amos Noyes Laboratory of Chemical Physics, ${ }^{\text {c) }}$ California Institute of Technology, Pasadena, \\ California 91125 \\ (Received 18 July 1978)
}

\begin{abstract}
One purpose of this paper is to present new studies on the effect of bandwidth and the coherence properties of the excitation source on the decay and the dephasing of isolated large molecules. A detailed study of the system pentacene in a $p$-terphenyl matrix is presented utilizing three different excitation sources, a single mode dye laser $(60 \mathrm{KHz}-6 \mathrm{MHz}$ bandwidth depending on the time scale of the experiment) a multimode dye laser ( $240 \mathrm{GHz}$ bandwidth), and an incoherent $\mathrm{N}_{2}$ flash lamp. Optical $T_{1}$ (the longitudinal relaxation time) and $T_{2}$ (the transverse relaxation time) are measured from the coherent and incoherent transients observed either in the forward direction of the laser or at right angles to the exciting beam. At $1.8 \mathrm{~K}$, the optical transition $\left({ }^{1} A_{18} \rightarrow{ }^{1} B_{2 k}\right)$ of pentacene in $p$-terphenyl exhibits four sites, the lowest of which at $16887 \mathrm{~cm}^{-1}$ has the following parameters: $T_{2}=44 \pm 2 \mathrm{~ns} ; T_{1}=24.9 \pm 2$ nsec, and $\mu=0.7 \pm 0.1 \mathrm{D}$. The transition moment $\mu$, is obtained directly from the optical nutation, which exhibits a Rabi nutation time $(\hbar / \mu \cdot \epsilon)$ of $27.3 \mathrm{~ns}$, and is corrected for the effect of the Lorentz local field inside the terphenyl crystal. The experiments presented here are categorized into two time regimes for theoretical analysis; a transient coherence regime where the observed decay is comparable with $(\mathrm{h} / \mu \cdot \epsilon)$ and $T_{2}$, and a steady-state coherence regime where transient dephasing is complete and the offdiagonal elements of the density matrix have decayed to their steady-state values in the presence of the field of amplitude $\epsilon$. Using the Wilcox-Lamb method, rate equations (with $T_{2}$ expressions) describing the population flow in the "complete" level structure of pentacene (ground $|0\rangle$, singlet $|p\rangle$, and triplet $\{\mid l\})$ are derived from the density matrix equations of motion. When these equations are averaged over the inhomogeneous width of the optical transition and the measured Gaussian transverse profile of our laser we obtain $T_{1 p 0}=24.9 \pm 2$ ns and $T_{1 p t}=15.7 \mu \mathrm{s}$, the time constants by which pentacene spontaneously decays to $|0\rangle$ or crosses over into $l_{\text {, }}$ as well as the averaged population at time $t$. In an effort to be complete, attention is placed upon the relationship between theory and the experimental findings. First, expressions for the OFID and nutation in the solid are presented for the pentacene case in order to relate $T_{1}, T_{2}$, and $\mu$ to the level structure. Second, at low temperatures $(1.8 \mathrm{~K})$, the origin of dephasing is identified as spontaneous emission from $p \rightarrow 0$ since experimentally $T_{2} \simeq 2 T_{1}$, in agreement with other work. At higher temperature, however, a strongly temperature dependent dephasing process with an onset at $3.7 \mathrm{~K}$ takes place. Armed with these observations we present a theoretical treatment of these distinct dephasing channels and their temperature dependences. A discussion regarding the influence of "accepting" phonon modes (either optical or acoustic) on optical dephasing is also given. The results indicate that the treatment of Jones et al, can (1) explain the observed temperature dependence of $T_{2}$ in pentacene; (2) distinguish dephasing as a result of scattering by acoustic phonons from that due to resonance or quasilocalized phonons with clear connections to gas and liquid state theories, but without invoking more than two optical levels; (3) explain both the level shift and line width changes as a result of "conventional" dephasing or dephasing by exchange mechanisms; and (4) relate the pure dephasing term to an anisotropy in the scattering amplitudes (between the ground and excited states in the system) which contribute largely to the homogeneous width of the transition. Optical site selection of these transitions is also reported and discussed in relation to vibrational relaxation and to both homogeneous and inhomogeneous broadenings. The studies of the homogeneous broadening of the vibronic origin (267 $\mathrm{cm}^{-1}$ ) indicate that vibrational relaxation is fast (ps) in the excited singlet manifold of pentacene. Finally from more than ten independent experiments including single and multimode excitation, on- and offresonance scattering, Zeeman effect and the transient decay as a function of excess energy in the molecule, a more complete picture of the pentacene level structure $\{|l\rangle\}$ is given. With this in mind, the influence of the laser bandwidth and coherence properties on state preparation and subsequent dephasing and decay is concluded. It is proposed that the slow decay, $(\sim 15 \mu \mathrm{s})$ observed during narrow-band excitation represents intersystem crossing to nearby triplet manifolds after the transient coherence of the $0 \leftrightarrow p$ subsystem is decayed. In addition, the decay of the primary state prepared in these experiments is not sensitive to the bandwidth or the correlation time of our excitation sources.
\end{abstract}

\section{INTRODUCTION}

Radiationless relaxation processes in large molecules have been under examination ever since their discovery and especially whenever new theoretical or experimen-

a) Present address: Dept. of Chemistry, University of Chicago, Chicago, IL, 60637.

b) Alfred P. Sloan Fellow.

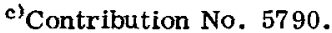

tal tools are made available. In these large molecules, where the density of vibrational modes at the optical excitation energy is very large, one usually finds that the lifetime of the excited state is shorter than the radiative lifetime (obtained from oscillator strength measurements) and the quantum yield is less than one, ${ }^{1}$ contrary to the behavior in small molecules discussed by Douglas. ${ }^{2}$ This paradox originates from a radiationless decay within the molecule, and was resolved by incorpo- 
rating different coupling mechanisms among the socalled Born-Oppenheimer (BO) singlet and triplet states. $^{3}$

In the theory $\mathrm{y}^{3-5}$ of radiationless transitions, the molecule has a primary or doorway state (e.g., a singlet state which carries most of the oscillator strength) $|p\rangle$ which may or may not couple to many isoenergetic vibronic levels $\{|l\rangle\}$ from the ground singlet state or nearby triplet manifolds. The coupling matrix elements $v_{p l}$ and the density of states will determine the routes of the nonradiative decay. In many ways this scheme resembles Fano's description ${ }^{6}$ of the autoionization of helium. However in molecules, ir reversible vibronic relaxation and radiative decay (optical $T_{1}$ processes; the longitudinal relaxation time) deactivate the excited level with no ionization or bond breakage. This irreversibility which has been discussed by Freed $^{7}$ and others ${ }^{3}$ requires that the vibrational levels in quasiresonance with $|p\rangle$ form an effective continuum. Under simple coupling conditions the line shape of the $|p\rangle$ state becomes Lorentzian in the energy domain. Thus if one prepares the $|p\rangle$ state, the homogeneous linewidth will be given by the sum of the radiative and nonradiative $T_{1}$ contributions.

The question that was first raised by Rhodes et al. ${ }^{8}$ and Robinson and Langhoff ${ }^{9}$ is: Do we really prepare the $|p\rangle$ state under all excitation conditions? This question has stimulated several theoretical investigations, ${ }^{3}$ and from them have emerged the conditions under which one may excite the BO singlet state or the molecular eigenstates $\psi_{m}$ which are linear combinations of the singlet and triplet (or hot ground singlet) states.

If the molecular eigenstates are prepared, then the radiative decay of these states will be different from the radiative decay of the $\mathrm{BO}$ singlet state according to the following relationships:

$$
\left|\psi_{m}\right\rangle=\alpha_{p m}|p\rangle+\sum_{l} \beta_{l m}|l\rangle
$$

and

$$
\left[T_{1 r}^{m}\right]^{-1}=\alpha_{p m} \alpha_{p m}^{*}\left[T_{1 r}^{p}\right]^{-1}+(\approx 0) .
$$

Hence, the radiative lifetime $\left(T_{1 r}\right)$ of the molecular eigenstates will be "scaled" according to the coeff $i-$ cients $\alpha$ 's which in turn depend on $v_{p l}$ and the energy difference between $|p\rangle$ and $\{|l\rangle\}$. It is precisely the coupling to the radiation field which makes the energy of the state $|m\rangle$ imaginary. Strictly speaking the states $\{|m\rangle\}$ therefore do not represent the true molecular eigenstates which diagonalize the following Hamiltonian:

$\mathfrak{H}=\mathfrak{H}($ singlet $)+\mathscr{H}($ triplets or hot singlets $)+\mathscr{H}($ interaction $)$.

In other words, the above semiclassical description says that because $|m\rangle$ couples to the radiation field continuum (or the nonradiative continuum), the monochromatic preparation makes the state evolve in time not only by the quantum mechanical stationary state characteristics but also by the Weisskopf-Wigner ${ }^{10}$ damping term; i.e.,

$$
\begin{aligned}
\langle m \mid m(t)\rangle & =\left\langle m\left|e^{-i x t / n}\right| m\right\rangle \\
& =\exp \left[-\frac{i}{\hbar}\left\{E_{m}-\frac{i}{2}\left(T_{1 r}^{m}\right)^{-1}-\frac{i}{2}\left(T_{1 n r}^{m}\right)^{-1}\right\} t\right],
\end{aligned}
$$

where $T_{1 n r}$ is the nonradiative $T_{1}$ decay time and $E_{m}$ is the real energy of state $\mid m$ ). (At this point we would like to emphasize that our use of the term "molecular eigenstate" is not rigorous because of the above reasons. Nevertheless, we shall use it to describe the states which are mixtures of the singlet $|p\rangle$ and $\left.\{|l\rangle\}_{.}\right)$ The spacing between $|m\rangle$ 's and their homogeneous linewidth will determine whether or not these states can be prepared in the laboratory, and whether or not the above theoretical description is adequate.

Experimentally, the first attempt to excite a large molecule with a narrow band ( $\mathrm{MHz}$ ) laser was made on pentacene about two years ago (henceforth referred to as $\mathrm{I}^{11}$ ). The experiments were performed hoping to answer the following questions:

(1) Do radiationless transitions depend on the nature of the exciting field?

(2) What is the exact nature of the state we excite?

(3) What is the influence of the ensemble optical coherence on the time evolution of the prepared state?

(4) To what extent does the correlation time of the light source influence the decay of the prepared state?

(5) Is there a threshold for an "ergodic" behavior in large molecules?

Our preliminary experiments on pentacene in a $p$ terphenyl host naturally did not answer all of these questions but they did shed some light on the effect of narrow band excitation on the observed (short and long) time resolved spectra. Furthermore, they provided the dephasing time (optical $T_{2}$; transverse relaxation time) following the nar row-band coherent excitation.

In this paper we provide a full account of our studies of the narrow and wide-band laser excitation done on pentacene in $p$-terphenyl at $1.7 \mathrm{~K}$ and above. A description of the effect of magnetic field, temperature, laser power, and frequency on the observed decay patterns is given and new results regarding the coherent transients are presented. We then relate these findings to the origin of optical pure dephasing (i. e., the process by which the prepared state loses its phase coherence) and radiationless decay in the solid at different temperatures. Finally, from more than ten independent experiments we present a more complete picture (different from I) for the excitation pathways in pentacene following the narrow - and wide-band excitation. This picture (a) does not support the preparation of $a$ molecular eigenstate, (b) explains the origin of optical dephasing $\left(T_{2}\right)$ and optical $T_{1}$, and (c) indicates that there are more than two states involved even in the narrowband laser excitation process.

The paper is outlined as follows. Section II presents the methodology and the physical description of the different transients (optical nutation, free induction decay, etc.) that are obtained from the coherent narrowband excitation, and will be used throughout the entire paper. Section III gives the experimental procedures 
TABLE I. Some nomenclature and definitions

\begin{tabular}{ll}
\hline Term & Morn-Oppenheimer states \\
\hline BO states & optical longitudinal relaxation time \\
$T_{1}$ & optical transverse relaxation time \\
$T_{2}$ & optical pure dephasing time \\
$T_{2}^{\prime}$ & optical free induction decay \\
OFID & incoherent resonance decay \\
IRD & electro-optic \\
EO & acousto-optic \\
AO & laser-acoustic diffraction spectroscopy \\
LADS & para-terphenyl: 0$\rangle$ \\
$p$-terphenyl & intersystem crossing \\
ISC & O)
\end{tabular}

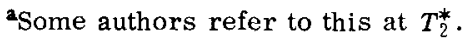

and data analysis. Section IV presents the relevant theory of dephasing for pentacene. Section V gives results and analysis of the observed coherent and incoherent transients. Finally, Sec. VI gives summary and conclusions and Table I presents some nomenclature used by us and others.

\section{OBJECTIVES AND METHODOLOGY}

The objectives of these experiments are to measure the decay $\left(T_{1}\right)$ and the dephasing $\left(T_{2}\right)$ of selectively prepared vibronic states of a large molecule like pentacene using broad (coherent and incoherent) and narrow-band excitation sources, and to determine the environmental effects as well as the effects of excess vibrational energy upon optical $T_{1}$ and $T_{2}$ processes. Pentacene was chosen because (a) it suits our single-mode laser which has an effective band width of $60 \mathrm{kHz}-16 \mathrm{MHz}$ depending on the time scale of the experiment, and (b) it has a level structure which might favor the formation of the states of Eq. (1) since triplet manifolds are close by the singlet state (see Ref. 13 and Table II).

To better understand what is meant by selectively prepared states one can refer to Fig. 1 where we show an inhomogeneously broadened optical transition represented as a Gaussian distribution of homogeneous groups each having a Lorentzian line shape whose width is given by $\left(\pi T_{2}\right)^{-1}$. For pentacene in a $p$-terphenyl host the inhomogeneous broadening is probably due to crystal field irregularities caused by strain and lattice defects. The homogeneous broadening on the other hand contains both the spontaneous decay ( $T_{1}$ contribution) and the contribution due to pure dephasing $\left(T_{2}^{\prime}\right)$. For the latter case the population of the state is unchanged and the "scattering", say by phonons, is elastic in nature.

Our experiments focus on unraveling the details of line-broadening mechanisms in pentacene by separating experimentally the contribution to the total homogeneous linewidth of the various $T_{1}$ relaxation processes from the $T_{2}^{\prime}$ processes. In this manner we can relate the measurements to the question raised earlier, namely, the effect of excitation bandwidth on the decay and dephasing of the prepared state. To obtain optical $T_{1}$ and $T_{2}$ following narrow-band excitation we have used the coherent transient methods or the method of emis sion detection of optical coherence. It is therefore important to understand the various phenomena that occur when large molecules interact with strong coherent fields, and to examine how the dynamics of the interaction provide the detailed molecular information we seek.

Before proceeding into a detailed discussion of our experiments and the theory required to understand the processes of interest, it is perhaps useful to make use of a general description that provides a physical explanation of the somewhat complicated and different terminology used throughout the paper for describing coherence experiments done by monitoring the intensity of the laser ${ }^{14}$ or the spontaneous emission. ${ }^{15}$

\section{A. Optical nutation}

When a group of molecules (say, $\alpha$ of Fig. 1) is brought into resonance with a strong coherent optical field the population can be completely driven from the ground state to the excited state in an oscillatory fashion as molecules alternately absorb and re-emit radiation coherently. This process known as optical nutation ${ }^{16}$ has an oscillation frequency (Rabi frequency) determined by $\mu$, the transition dipole moment and $\epsilon$ the laser field amplitude. A nutation is damped due to inhomogeneous broadening and the nonuniform spatial in-

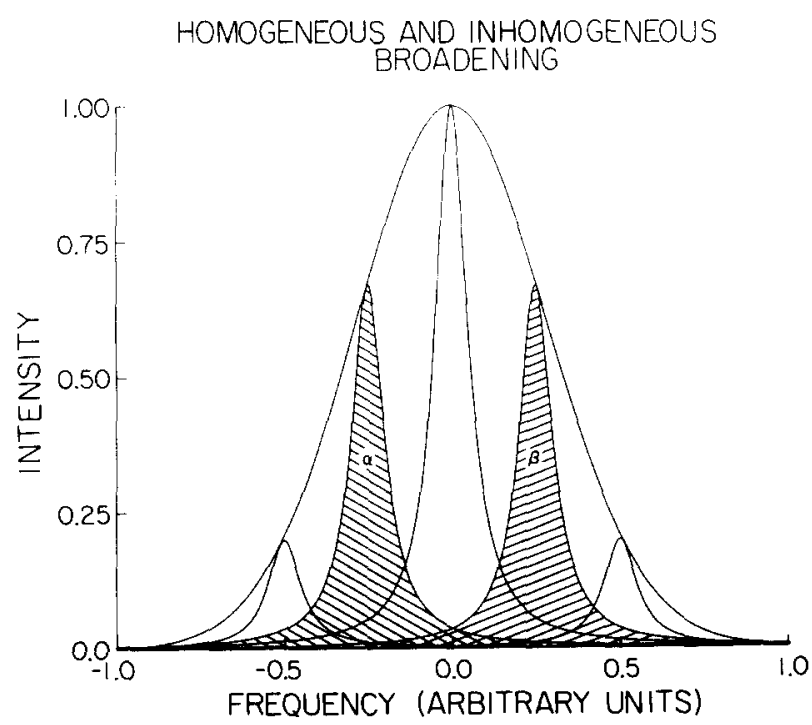

FIG. 1. Inhomogeneous broadening of an optical transition in a solid. Shown is a Gaussian distribution of homogeneously broadened molecular groups; each having a Lorentzian line shape. The shaded areas, $\alpha$ and $\beta$, refer to distinct groups of molecules selected in the narrow-band coherence experiments. 
TABLE II. Pentacene optical properties. ${ }^{2}$

\begin{tabular}{|c|c|c|c|c|c|c|c|}
\hline Phase & State & Origin $\left(\mathrm{cm}^{-1}\right)$ & $\begin{array}{l}\text { Extinction } \\
\text { coefficient } \\
\times 10^{-3}\end{array}$ & $\begin{array}{l}\text { Oscillator } \\
\text { strength }\end{array}$ & Lifetime (ns) & Assignment & Ref. \\
\hline & $s_{0}$ & & & & & ${ }^{2} A_{1}^{-}$ & $\mathrm{b}$ \\
\hline vapor $(580 \mathrm{~K})$ & $s_{1}$ & 18900 & & & $6.1^{1}$ & ${ }^{1} B_{2 \mu}^{+}$ & c \\
\hline solution ( $n$-heptane) & & 17100 & 12 & 0.08 & & & b \\
\hline $\begin{array}{l}\text { solid ( } p \text {-terphenyl) } \\
\quad(1.8 \mathrm{~K})\end{array}$ & $S_{1}$ & $\left\{\begin{array}{l}17065 \\
17006 \\
16887 \\
16883\end{array}\right\}^{\mathrm{d}}\left\{\begin{array}{l}17069 \\
17009 \\
16891 \\
16887\end{array}\right\}^{\mathrm{m}}$ & & & $\begin{array}{c}9.7^{\mathrm{j}} \\
9.7^{\mathrm{j}} \\
25.7^{\mathrm{j}} \\
25.7,^{\mathrm{g}} 23.5^{\mathrm{k}},^{\mathrm{k}} 24.5^{1}\end{array}$ & $\begin{array}{l}O_{4} \\
O_{3} \\
O_{2} \\
O_{1}\end{array}$ & \\
\hline solution ( $n$-heptane) & $S_{2}$ & 24000 & 0.6 & & & ${ }^{1} B_{3 u}^{-}$ & $b$ \\
\hline solution ( $n$-heptane) & $s_{3}$ & 32300 & 300 & 2.2 & & ${ }^{1} B_{3 w}^{+}$ & b \\
\hline calculation & $T_{1}^{(0)}$ & 6400,7800 & & & $\begin{array}{l}\text { (vapor) } 33000^{\mathrm{c}} \\
\text { (solution) } 70000^{\mathrm{B}}\end{array}$ & ${ }^{3} B_{2 \mathrm{u}}^{+}$ & $e, f$ \\
\hline solid (tetracene) & $T_{1}^{(0)}$ & 6500 & & & & & $\mathrm{~h}$ \\
\hline calculation & $T_{2}^{(0)}$ & 17000,21700 & & & & ${ }^{3} B_{3 u^{\prime}}^{+},{ }^{3} B_{3 \mu}^{-}$ & f \\
\hline $\begin{array}{l}\text { solution (benzene) } \\
\quad(323 \mathrm{~K})\end{array}$ & $T_{3}$ & $T_{1}+19820$ & 120 & 0.7 & & ${ }^{3} B_{18}^{-}$ & g \\
\hline vapor $(580 \mathrm{~K})$ & $T_{3}$ & $T_{1}+21500$ & & & & & c \\
\hline $\begin{array}{l}\text { solution (benzene) } \\
\quad(323 \mathrm{~K})\end{array}$ & $T_{4}$ & $T_{1}+25500$ & 3 & $<0.005$ & & ${ }^{3} A_{\mathrm{ig}}^{-}$ & g \\
\hline
\end{tabular}

The axis system adopted here is: $x$, long axis; $y$, short axis; and $z$ is the out of plane axis.

$\mathrm{b}_{\mathrm{E}}$. Clar, Aromatische Kohlenwasserstoffe (Verlag, Julius Springer, Berlin, 1941); H. B. Klevens and J. R. Platt, J. Chem. Phys. 17, 470 (1949).

${ }^{c}$ B. Soep, Chem. Phys. Lett. 33, $108(1975)$.

${ }^{d} J$ J. H. Meyling and D. A. Wiersma, Chem. Phys. Lett. 20, 383 (1973).

${ }^{8}$ R. Pariser, J. Chem. Phys. 24, 250 (1956).

${ }^{\text {f}}$ D. R. Kearns, J. Chem. Phys, 36, $1608(1962)$.

${ }^{8}$ C. Hellner, L. Lindquist, and P. C. Roberge, Faraday Trans. II 68, 1128 (1972).

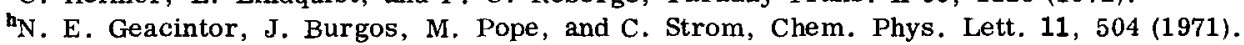

'S. Okajima and E. C. Lim, Chem. Phys. Lett. 37, 403 (1976).

${ }^{\mathrm{J}} \mathrm{N}$. J. Bridge, University of Kent, private communication; $T=4.2 \mathrm{~K}$.

${ }^{k_{T}}$. J. Aartsma, J. Morsink, and D. A. Wiersma, Chem. Phys. Lett. 47, $425(1977) ; T=1.8 \mathrm{~K}$.

${ }^{1}$ Our measurement (LADS) was independent of temperature in the region $1.8 \rightarrow 4.2 \mathrm{~K}$.

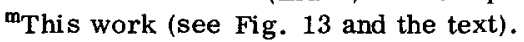

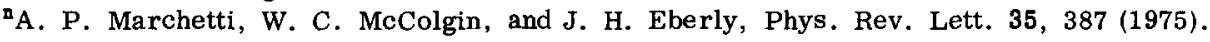

${ }^{\circ}$ These $T_{1}$ and $T_{2}$ refer to the first and second triplet states of pentacene, and should not be confused with $T_{1}$ and $T_{2}$ relaxation times.

tensity distribution of the laser as well as the molecular dephasing processes. From this coherent transient which occurs at the leading edge of the laser pulse one can determine $\mu$ and estimate $T_{2}$. Under certain conditions in solids, $T_{2}$ can be determined.

\section{B. Optical free induction decay (OFID)}

As a group of molecules initially on resonance with a coherent field (not necessarily a strong field) is suddenly switched off resonance they will emit initially in phase. ${ }^{17}$ If, as shown in Fig. 1 , the laser frequency is switched from being on resonance with the homogeneous group $\alpha$ to group $\beta$, then the molecules emitting coherently (from $\alpha$ ) can beat against the new laser frequency $\left(\omega_{\beta}\right)$ if a square-law detector is used for moni- toring the transmitted laser beam. The beat pattern will decay as the molecules dephase and as with the optical nutation, the FID contains contributions from inhomogeneous and power broadening processes. Extrapolated to zero laser power gives the decay time $T_{2}$ directly.

Now, since the laser is brought into resonance with group $\beta$ an optical nutation can occur (at $\omega_{\beta}$ ) at the same time but if the laser power is low enough only the OFID pattern from $\alpha$ will be observed.

\section{Incoherent resonance decay}

Up to this point we have discussed coherent absorption and coherent emission processes which are usually observed along the laser beam. One can also observe 
these coherent signals utilizing the incoherent (spontaneous) emission ${ }^{15}$ at right angles to the exciting beam and from it obtain optical $T_{1}$ and $T_{2}$.

If the emission intensity is measured as the single mode laser frequency rapidly changes from $\alpha$ to $\beta$ then one should see the superposition of two processes occurring simultaneously. First, $\alpha$ molecules will decay (by $T_{1}$ ) since they are now off resonance. At the same time, $\beta$ molecules are being driven coherently (i.e., nutating) by the laser field toward some equilibrium population distribution. Thus, the observed signal should show an initial rapid buildup and nutation transient (due to $\beta$ molecules) and a decay (due to $\alpha$ molecules. We have used IRD to denote the process of incoherent resonance decay.

\section{EXPERIMENTAL}

\section{A. Sample preparation}

p-Terphenyl (scintillation grade) was purchased from Eastman Organic Chemicals and zone refined for eighty passes. Pentacene (Aldrich Chemical Company) was twice vacuum sublimed. Dilute single crystals $\left(10^{-5}-\right.$ $10^{-7} \mathrm{~m} / \mathrm{m}$ ) of pentacene in $p$-terphenyl were grown from the melt using standard Bridgman techniques. Crystals (1-3 $\mathrm{mm}$ thick) were cut parallel to the $a b$ cleavage plane and mounted in most experiments with the crystal $b$ axis parallel to the polarization direction of the exciting laser.

\section{B. Cryogenics}

The crystals were suspended directly in a liquid helium filled glass Dewar. Temperatures down to 1.7 $\mathrm{K}$ were obtained by pumping on the helium reservoir with a Sargent-Welch (Model 1374) vacuum pump. The temperature was monitored with a calibrated carbon glass resistor (Lakeshore Cryotronics CGR-1-1000) mounted on the sample holder near the crystal and by measuring the vapor pressure of helium with calibrated Wallace and Tiernan pressure gauges.

\section{Laser excitation sources}

Three different laser sources were used for the excitation of pentacene in $p$-terphenyl. For broad band excitation, a pulsed tunable dye laser (Molectron DL 200) pumped by a nitrogen gas laser (Molectron UV 400) or a multimode $\mathrm{cw}$ dye laser were used. The $\mathrm{N}_{2}$ pumped dye laser has a frequency bandwidth of $18 \mathrm{GHz}$, a peak power of $40 \mathrm{~kW}$, and a pulse duration of $5 \mathrm{~ns}$. The multimode $\mathrm{cw}$ dye laser has a bandwidth of $240 \mathrm{GHz}$ and up to $1 \mathrm{~W}$ output power.

For narrow-band excitation (see Fig. 2) we used a single-mode tunable jet-stream cw dye laser (Spectra Physics 580A) pumped by an argon ion cw gas laser (Spectra Physics 171). To obtain single-mode operation, the dye laser has three intracavity etalons with different free spectral ranges (FSR). One of the etalons
(2 $\mathrm{mm}$ air spaced) is temperature regulated and piezoelectrically tunable. The other two are solid etalons of $0.17 \mathrm{~mm}$ and $\approx 1.7 \mu$ thickness, respectively.

For all of these experiments Rhodamine $6 G$ is used. The maximum laser power of the linearly polarized single mode is $100 \mathrm{~mW}$ in a Gaussian beam (Fig. 3) with a half-width $(1 / e)$ of $0.31 \pm 0.01 \mathrm{~mm}$ providing a maximum intensity (unfocused) of $33 \mathrm{~W} / \mathrm{cm}^{2}$. The transverse field profile of the single mode beam was measured using a photodiode mounted on a micrometer controlled translation state $(4 \mu$ resolution). Slits $(\approx 50 \mu)$ were attached to the photodiode thus limiting its aperture. The results in Fig. 3 were obtained by scanning the photodiode across the beam. The Gaussian profile seen here is what one expects for a TEM $\mathrm{T}_{00}$ beam.

The frequency bandwidth is determined by jitter and drift due to jet instabilities, temperature fluctuations, and vibrations. With a combination of techniques we were able to reduce the jitter (long term) of the single mode to $\pm 3 \mathrm{MHz}$ without active frequency feedback loops. These techniques involved (1) careful control of the dye solution temperature using a regulated bath; (2) independent air conditioning for the lab providing room temperature stability to $\pm 1^{\circ} \mathrm{C}$; (3) use of a vibration isolation optical table to mount all laser components; (4) soundproofing of the dye laser with a tight fitting enclosure, and (5) amplitude stabilization of the single mode by a feedback circuit.

\section{The laser frequency switching and laser-acoustic diffraction techniques}

To obtain nanosecond time resolution with the $\mathrm{cw}$ narrow-band laser we employ either electro-optic (EO) frequency modulation or laser-acoustic diffraction. (We call the latter technique LADS $^{18}$ for Laser Acoustic Diffraction Spectroscopy to avoid confusion with another technique: opto-acoustic spectroscopy developed by Robin and his co-workers. ${ }^{18}$ ) For EO frequency modulation we modified the laser cavity to accept an $A D^{*} P$ crystal. Frequency switching is accomplished by supplying voltage pulses to the EO crystal which alter its index of refraction and the optical path length. Thus, for the duration of the voltage pulse, the frequency of the laser can be changed from $\omega_{0}$ (the peak frequency of the transition resonance) to a new frequency $\omega$. This EO switching technique was first described by Yariv ${ }^{20}$ and later by Kiefer et al. ${ }^{21}$ for CdTe modulators. Hall ${ }^{22}$ was the first to demonstrate the frequency switching technique to observe a coherent transient (FID) in methane in the IR. He used a $\mathrm{LiNbO}_{3}$ modulator to frequency switch a $\mathrm{He}-\mathrm{Ne}$ laser operating at $3.39 \mu$. Shoemaker and Van Stryland ${ }^{23}$ discussed how this technique would be useful for observing the nutation and thus for measuring transition moments. Brewer and Genack $^{24}$ were successful in extending the technique to the visible region and measured transients in $I_{2}$. Since then this laser frequency-switching method has allowed one to observe coherent transients in gases, ${ }^{24,15}$ solids, $^{25,26}$ and molecular beams. ${ }^{27}$ In addition to the above-mentioned technique we have utilized the $\operatorname{IRD}^{15}$ method to monitor the coherence of the ensemble ex- 


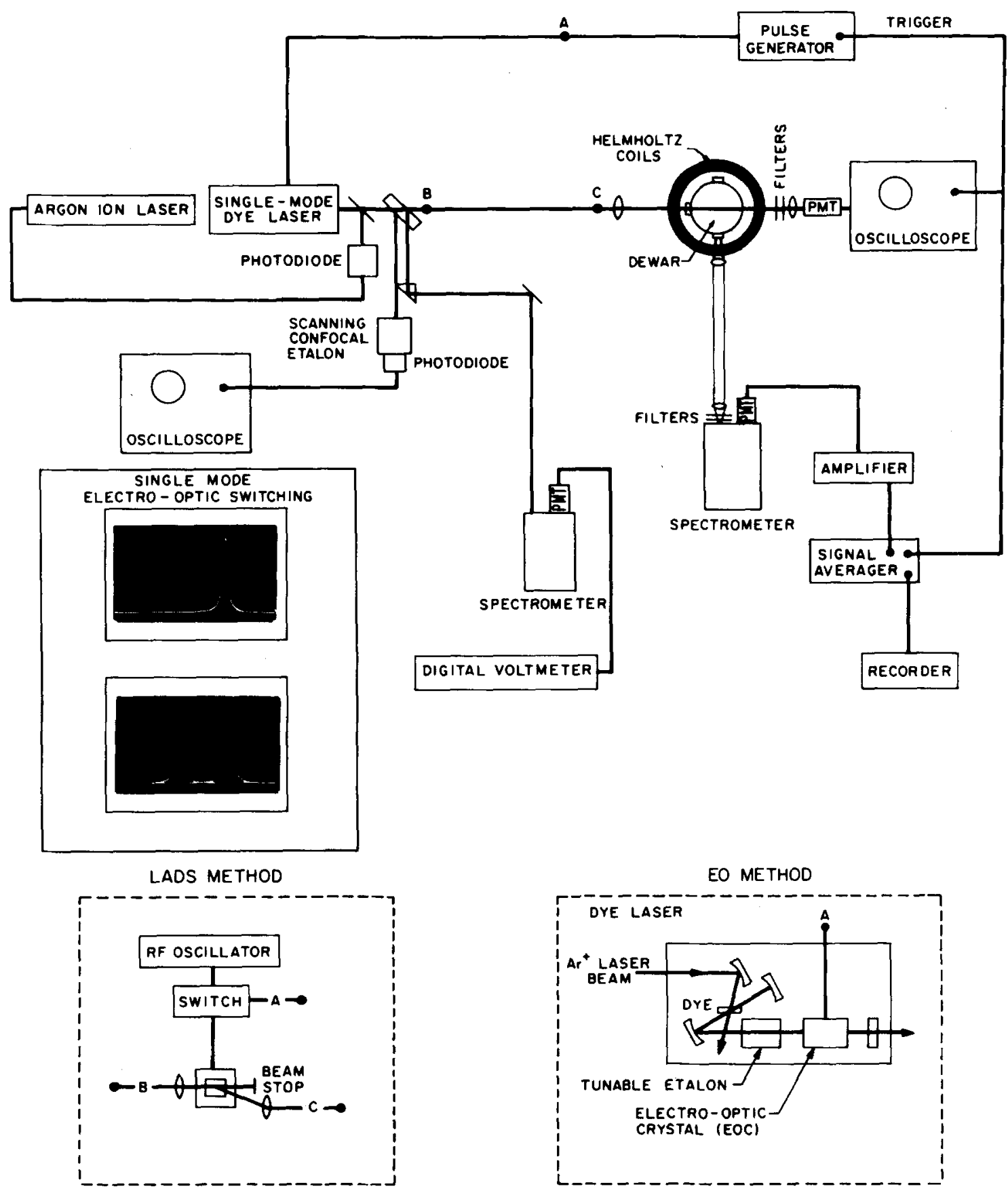

FIG. 2. A schematic diagram of the experimental arrangements used for EO and LADS experiments. All laser components are mounted on a vibration-isolation optical table. The electro-optic switching process shown in the insert is monitored by synchronizing the scanning confocal etalon with the voltage pulse supplied to the AD*P crystal. The other insert depicts the method of LADS.

cited by a narrow-band laser. In this method, the $\mathrm{EO}$ crystal is also intracavity.

Typically, the dispersion of the nonlinear EO crystal gives a $60 \mathrm{MHz}$ frequency shift within the inhomogeneous resonance of the optical transition (width $>\mathrm{GHz}$ ) for voltage pulses of $100 \mathrm{~V}$. To switch the laser frequency further (which would be necessary in systems with larger homogeneous linewidths), fast high-voltage pulses are required. In practice, these pulses are hard to obtain-especially at high repetition rates and when multiple pulse (photon echo) experiments are desired. Fur- thermore, there is a limit on the maximum frequency shift possible (maintaining stable single-mode output) which is determined by the cavity mode spacing ( 400 $\mathrm{MHz}$ ). When one finds it difficult to switch out of the homogeneous linewidth (i.e., off-resonance) with the EO method, then one can utilize the LADS method.

The LADS technique for observing coherent transients was reported recently by Orlowski et al. ${ }^{18}$ An extra cavity acousto-optic modulator is used to diffract the laser beam to and from the sample. Thus, instead of switching the frequency of the single-mode laser off 
SINGLE-MODE LASER TRANSVERSE BEAM PROFILE

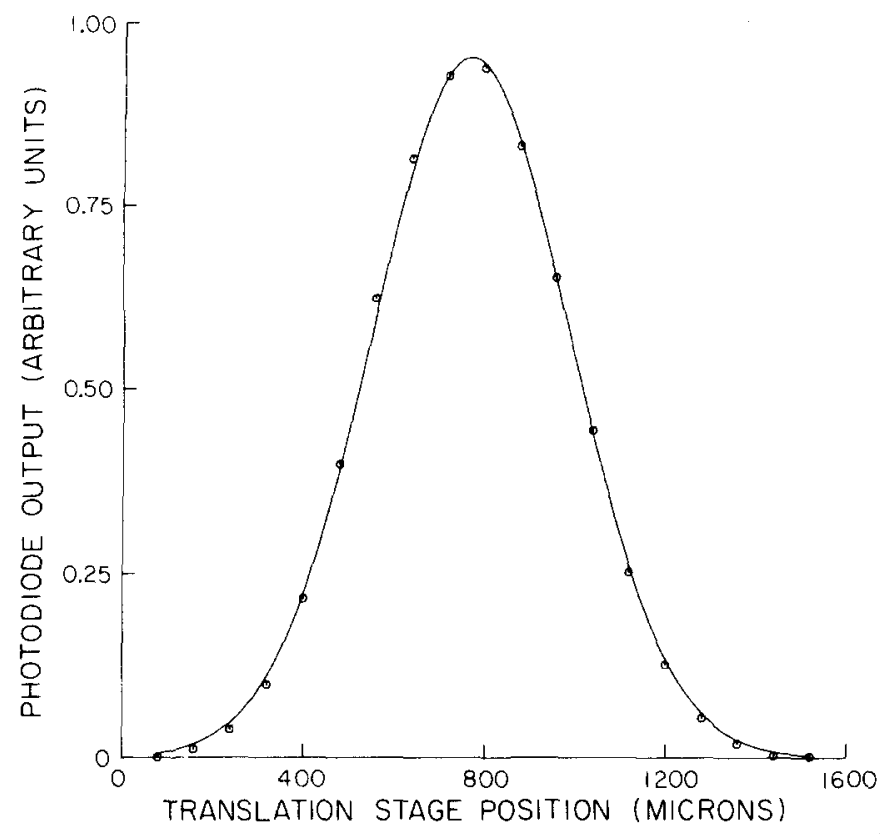

FIG. 3. The transverse beam profile of the single-mode laser. Experimental data (circles) obtained by scanning a carefully aperatured $(50 \mu)$ photodiode across the laser beam are fit to a Gaussian whose $1 / e$ half-width is $0.31 \pm 0.01 \mathrm{~mm}$. The excellent fit to a Gaussian profile confirms the $T E M_{00}$ nature of the laser beam. This will be important for obtaining the transition moment from optical nutation experiments (see text).

resonance within the inhomogeneous line we effectively turn the laser "off". This technique relies on the acousto-optic effect $\mathrm{t}^{28}$ and can be described as follows. The single-mode beam is focused upon a quartz crystal which has a thin film transducer bonded to it. rf pulses excite phonons in the transducer creating traveling acoustic waves in the crystal which diffract the incident beam (up to $50 \%$ ) at the Bragg angle and shift its frequency by the acoustic phonon frequency (470 $\mathrm{MHz}$ ). Thus, the diffracted beam is deflected into the sample for the duration of the rf pulse. The characteristics of the laser pulse this technique provides are determined by (1) the rf pulse width and its rise/fall times and (2) the transit time of the acoustic wave across the focused laser beam in the crystal. We have measured laser pulse rise/fall times of $3 \mathrm{~ns}$ (1/e time) using a $75 \mathrm{~mm}$ focal length lens to focus the laser beam into the crystal.

There are advantages to both of the techniques described above. The EO technique is most useful for observing OFID since extremely sensitive heterodyne detection techniques can be employed which exploit the fact that the laser is off-resonance and can beat with the coherent emission from the sample. The LADS methods offers the following advantages. First, the decay of offresonance molecules is not superimposed on the buildup transients of on-resonance molecules (which occurs with the EO technique) because the laser is effectively shut off except for the duration of the pulse. This is especially important for large molecules with a high density of excited states. In these systems the complicated level structure can make the interpretation of transient spectra difficult. The LADS method removes the influence of off-resonance effects and thus simplifies the spectra. Secondly, the optical pulses are generated by supplying low-voltage (TTL level) pulse trains to an rf oscillator instead of the high-voltage pulses that may be required by the EO technique. Finally, one can obtain high sensitivity as with heterodyne techniques by detecting the spontaneous emission at right angles to the exciting laser beam. With the proper pulse sequences, one can dirertly measure both $T_{1}$ and $T_{2} \cdot{ }^{18}$

\section{E. Magnetic field experiments}

Several experiments were performed with samples in the presence of an external magnetic field. Two coils were placed around the tail of the Dewar at the Helmholtz spacing such that the field direction was parallel to the direction of laser polarization. With 22 amps of current supplied by a regulated dc power supply we measured a field strength of 900 Oersted at the sample using a Bell Gaussmeter and a calibrated Hall effect probe. The field was homogeneous at the crystal to better than $1 \%$.

\section{F. Experimental procedure}

The apparatus used for the EO and LADS experiments is shown schematically in Fig. 2. The output of the single-mode laser is amplitude stabilized and split $(8 \%)$ to allow continuous monitoring of laser frequency and single-mode operation using a $0.5 \mathrm{~m}$ spectrometer and a scanning confocal etalon, respectively.

For LADS experiments the modulator was placed in the optical path such that the diffracted beam continues on to the sample while the transmitted beam was blocked. Thus, the sample was excited only when an rf pulse was supplied to the AO crystal.

Coherent transients along the laser beam were detected using fast biased photoiodes (HP 5082-4203) and/ or a modified RCA IP28 photomultiplier. ${ }^{29}$ In some experiments (OFID) polarizers were used on either side of the Dewar to ensure that only light polarized with the laser reached the detector.

For some experiments the transients were displayed directly on a sampling scope, however, signal averaging was also done using a scanning-gate boxcar integrator (PAR Model 162).

Emission from the sample was detected at right angles to the exciting beam using a shielded ( $\mathrm{rf}$, magnetic, electrostatic), cooled photomultiplier (EMI 9558). For detecting very fast transients on the emission we used the modified IP28 photomultiplier which has a bandwidth greater than $1 \mathrm{GHz}$. Sharp-cut glass filters were used to block the exciting wavelength. In most experiments the total emission from the sample was monitored, however, several experiments were performed using a $0.5 \mathrm{~m}$ (or $0.75 \mathrm{~m}$ ) spectrometer to resolve individual vibronic lines. The output of the PMT was amplified and fed to the boxcar integrator for signal averaging. 
Absorption experiments were performed by using a Tungsten $(1000 \mathrm{~W})$ or a xenon $(200 \mathrm{~W})$ lamp, together with the appropriate filters. For high-resolution measurements a Spex (1-14018) double monochromator equipped with holographic gratings (resolution $=0.09$ $\mathrm{cm}^{-1}$ ) was used. In some experiments, a Fabry-Perot interferometer (Burleigh RC 110) in front of a $0.5 \mathrm{~m}$ Jarrell-Ash spectrometer was used for scanning the line shape profile.

\section{G. Data treatment}

\section{Temperature-dependence measurements}

The temperature dependence of the optical nutation signal (initial amplitude) and the IRD signal were determined using the following carefully controlled procedure. For the optical nutation experiment the dc signal and the transient initial amplitude were monitored at each temperature. Detector linearity was checked using calibrated neutral density filters. As the temperature rose above the $\lambda$ point of liquid helium and boiling started, the dc signal decreased one order of magnitude due to scattering from helium bubbles. Amplifier gain was increased at this point. All data were corrected for the dc signal strength which appeared to decrease slightly as the temperature was raised. Since the transients observed in our experiments were insensitive to temperature until $\approx 3 \mathrm{~K}$ the $\lambda$ point $(2.17 \mathrm{~K})$ does not represent a problem in determining the onset of the temperature effect.

For the IRD experiments, the entire decay signal was measured at each temperature. The PMT current (dc) was kept constant (within the detector's linear region) throughout the range of temperatures studied. We observed an increase in the dc emission intensity (factor of 3) when the temperature was increased from 2.17 to $4.2 \mathrm{~K}$. Similar temperature dependences were obtained for both the optical nutation and IRD experiments.

\section{Zeeman measurements}

IRD experiments performed with external magnetic fields of up to 900 Oersted were compared very carefully with their zero-field counterparts using the following procedure. First, an IRD decay and dc emission intensity were measured at zero field. Then, the field was turned on and another decay (and intensity) were recorded. Finally, we immediately repeated the zero field experiment to insure reproducibility. Because the magnetic field effect on the observed decay was small $(\approx 15 \%)$ we repeated this cycle more than twenty times with several different crystals, concentrations, and orientations. In all experiments which utilize a shielded photomultiplier reproducibility was excellent, however, the magnitude of the effect was very sensitive to slight changes [one cavity mode ( 390 $\mathrm{MHz}$ ) or less] in laser frequency. One should note that the effect of a small dc magnetic field on the photomultiplier current might result in small changes in the dc signal level but will not affect the dynamic response of the tube. More discussion of the Zeeman experiments will be presented later.

\section{Computer fitting procedures}

Computer assisted nonlinear least squares regression analyses were performed to fit all of the IRD data as well as the optical nutation and the OFID. To avoid some of the ambiguities associated with these techniques (e.g., making initial estimates of parameters which may be biased or which may lead to only a local minimization of error) most of the data was fit in a twostep procedure. First, we utilized an eigenfunction expansion method developed by Provencher ${ }^{30}$ for determining the best exponential decay parameters (rate constants and coefficients) for the IRD transients [discussed later in Eqs. (43) and (46)]. This method requires no initial guesses and provides cross correlation coefficients as well as confidence limits. From the values of the coefficients of the exponentials we were able to make good initial guesses for the rate constants needed in the steady-state coherence model of Sec. IV B. Then, we used a more conventional (and more general) procedure for nonlinear least squares regression analysis using an algorithm developed by Marquardt. ${ }^{31}$ Although our program handles up to 50 parameters, only two parameters were simultaneously varied (for the IRD fits) to get solutions that converged upon the same values for several different starting points. Thus, unique solutions can be assumed safely in most cases. Similar procedures were adopted for treating the results of other transients, and thus the program, which is stored in the Caltech computer library, was used routinely for analyzing all the data reported in this paper.

\section{THEORY OF DEPHASING : PENTACENE}

Pentacene, which vibrates in 102 different optical molecular modes, has approximately $3 \times 10^{14}$ vibrational levels per wavenumber near the energy of the |p) state. ${ }^{32}$ This implies that the molecule belongs to the "statistical limit" in the sense that the levels interacting with $|p\rangle$ form essentially a cont inuum. How ever, the presence of a nearby triplet manifold might make the interaction between the sparse levels of the triplet state and the $|p\rangle$ state a discrete one, thus resulting in the formation of $|m\rangle$ states. Furthermore, not every state out of the $10^{14}$ levels has the appropriate Franck-Condon and/or symmetry properties for coupling with $|p\rangle$. If this coupling between $|p\rangle$ and quasiresonant levels is operative and the homogeneous linewidth of the resulting states is less than their spacing, we expect to locate $|m\rangle$ 's which as mentioned before have different $T_{1}$ decay times from that of the $|p\rangle$ state.

In the statistical limit, ${ }^{5}$ it has been shown, ${ }^{3,33}$ that following the excitation into $|p\rangle$, the decay will exhibit a fast component with a rate of $(2 \pi / \hbar) v_{p l}^{2} \rho\left(E_{p}\right)$ and a slow component $\left(T_{1 m}^{-1}\right)$ in addition to a complicated beat pattern due to interference effects ${ }^{34}$ similar to those of quantum beat phenomena. In the sparse limit the situation is different and depends on how many $|m\rangle$ states the light source spans as well as the correlation time of the source.

If $|m\rangle$ is excited say by a coherent narrow-band source, then the description of dephasing at any time $t$ 


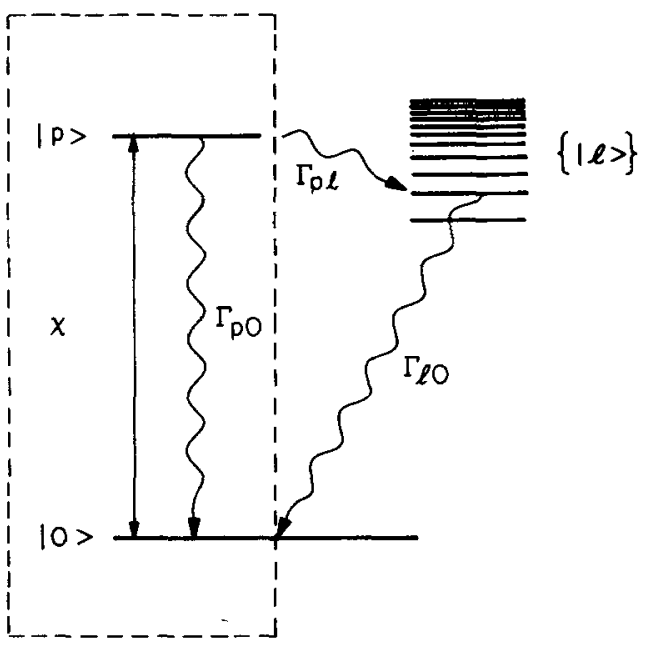

FIG. 4. A schematic diagram of the level structure in pentacene showing important decay channels. The levels $|0\rangle$ and $|p\rangle$ refer to the ground and first excited electronic states, respectively, while $\{|l\rangle\}$ is a manifold of effective levels that interacts with the two-level system enclosed in dashed lines. The nature of the $\{|l\rangle\}$ manifold is unspecified and all the rate constants are defined in the text (see Sec. IV).

is that of a two-level system provided there are no cross relaxations among $\{|m\rangle\}$. The treatment for the interaction between coherent light and such two-level systems is a standard one and can be found in several articles and textbooks. ${ }^{35}$ If, on the other hand, $|p\rangle$ is prepared as depicted in Fig. 4, then we have more than two levels to treat, and the description of the time evolution of the excited state crucially depends on the time scale of the experiment.

As we shall see from the experiments done on pentacene, the picture shown in Fig. 4 can be simplified considerably if one knows optical $T_{1}, T_{2}$, and the Rabi frequency. Of course, the definitions of $T_{1}$ and $T_{2}$ in such a complex level structure require some care since a genuine dephasing mechanism might be washed out by changing representations or conversely an artificial dephasing process may be introduced by changing the number of degrees of freedom

For the pentacene case, we define the following rates for the different pathways:

$$
\begin{aligned}
& \Gamma_{p 0}=T_{1 p 0}^{-1}=\text { total decay rate of }|p\rangle \text { to }|0\rangle \\
& \Gamma_{p l}=T_{1 p l}^{-1}=\text { total decay rate of }|p\rangle \text { to }\{|l\rangle\} \\
& T_{1 p}^{-1}=T_{1 p l}^{-1}+T_{1 p 0}^{-1}=\text { total rate for the depletion of }|p\rangle \\
& \begin{array}{l}
\Gamma_{l 0}= \\
\text { population }
\end{array} T_{1 l 0}^{-1}=\text { total decay rate of } l \text { into }|0\rangle \\
& T_{10}^{-1}=T_{1 p 0}^{-1}+T_{1 l 0}^{-1}=\text { total feeding rate to }|0\rangle \\
& \chi=\text { "pump" rate } \\
& \Gamma=T_{2}^{-1}=\text { total dephasing rate of the two levels }|p\rangle \text { and } \\
& \quad \quad 0\rangle \text { that will be discussed later in Eqs. (10) } \\
& \quad \text { and (11). } \\
& T_{2}^{\prime-1}=\text { rate of pure dephasing (with no } T_{1} \text { terms) of the } \\
& \text { two levels }|p\rangle \text { and }|0\rangle .
\end{aligned}
$$

In what follows we provide the theoretical analysis that will be used later in all of the experiments done on pentacene. These experiments span different time regimes, and effectively probe the excited population either in the $|0\rangle$ and $|p\rangle$ levels (the levels in the dashed rectangle of Fig. 4) or in the entire (statistically closed) level structure after the phase coherence of $|0\rangle$ and $|p\rangle$ achieves a steady state. Thus we have two extreme limits which we will term the transient coherence and steady-state coherence regimes, respectively. If the observed transient decay time $\tau_{0}$ (observed say by monitoring the population of $|p\rangle)$ is much longer than $(\hbar / \mu \cdot \epsilon)$ and $T_{2}$, then the steady-state coherence regime can be used assuming that on the $\tau_{0}$ time scale all correlations among $\{|l\rangle\}$ do not exist; otherwise one may use the decoupling scheme of Grigolini and Lami. ${ }^{36}$ On the other hand, if $\tau_{0}$ is comparable to $(\hbar / \mu \cdot \epsilon)$ the transient coherence coupling regime will be recovered.

\section{A. Transient coherence regime: $T_{0} \approx T_{2}$}

In this limit the rate of optical pumping $(\chi)$ is very large and the dephasing is reaching a steady state value before the channel to $\{|l\rangle\}$ is opened. In effect the two levels are therefore decoupled from the rest of the (many) molecular levels. To describe the dephasing of the two levels on such time scales, we shall follow the standard procedure of using the density matrix and the representation of Feynman, Vernon, and Hellwarth (FVH). ${ }^{37}$

First, we write the complete Hamiltonian for the system as a sum of the zero-order Hamiltonian and an interaction term signifying the coupling of the two molecular states by the field:

$$
\mathcal{H}=\frac{\hbar \omega_{0}}{2} \sigma_{3}-\mu \cdot \epsilon \sigma_{1} \cos \omega t .
$$

Here $\sigma_{1}$ and $\sigma_{2}$ are the Pauli matrices, and $\omega_{0}$ and $\omega$ are the transition and laser frequency, respectively. In the laboratory frame, the equation of motion for the system is therefore given by

$$
i \hbar \dot{\rho}^{\prime}=\left[\mathfrak{F}, \rho^{\prime}\right],
$$

where $\rho^{\prime}$ is the density matrix and the prime is used to designate the laboratory frame representation.

By going into a rotating frame of reference (at $\omega$ ) one obtains

$$
i \hbar \dot{\rho}=\left[\mathfrak{H}_{r}, \rho\right] \text {, }
$$

where

$$
\mathcal{H}_{r}=\mathrm{U}^{-1} \mathfrak{H C}-\frac{\hbar \omega}{2} \sigma_{3} \approx \frac{\hbar \Delta}{2} \sigma_{3}-\frac{\mu \cdot \epsilon}{2} \sigma_{1} .
$$

Equation (8) was obtained using the unitary transformation $\mathrm{U}=e^{-1 \omega \sigma_{g} t / 2}$ and the following identity:

$$
e^{i \omega \sigma_{3} t / 2} \sigma_{1} e^{-i \omega \sigma_{3} t / 2}=\sigma_{1} \cos \omega t-\sigma_{2} \sin \omega t .
$$

Terms oscillating at $2 \omega$ were neglected and $\Delta$ is defined as $\omega_{0}-\omega$. Given Eqs. (7) and (8) one can show that the equations of motion for $\rho$ can be written, after adding phenomenological relaxation terms for pentacene, as: 


$$
\begin{aligned}
& \dot{\rho}_{00}=\frac{1}{T_{1 p 0}} \rho_{p p}+\frac{1}{2} i \chi\left(\rho_{p 0}-\rho_{0 p}\right) \\
& \dot{\rho}_{p p}=\frac{-1}{T_{1 p 0}} \rho_{p p}+\frac{1}{2} i \chi\left(\rho_{0 p}-\rho_{p 0}\right) \\
& \dot{\rho}_{0 p}=\left(-\frac{1}{T_{2}}+i \Delta\right) \rho_{0 p}+\frac{1}{2} i \chi\left(\rho_{p p}-\rho_{00}\right) .
\end{aligned}
$$

Here $T_{1 p 0}$ is the spontaneous emission lifetime of the upper level, $\chi=\mu \cdot \epsilon / \hbar$ and

$$
\frac{1}{T_{2}}=\frac{1}{2 T_{1 p 0}}+\frac{1}{T_{2}^{\prime}}=\frac{1}{2 T_{1}}+\frac{1}{T_{2}^{\prime}} .
$$

The quantity $\left(T_{2}^{\prime}\right)^{-1}$, the pure dephasing rate, represents elastic interactions with phonons in the solid which at very low temperatures approaches zero. Thus, $T_{2}$ $=2 T_{1 p 0}$ in the absence of dephasing processes in the system and when the level structure ${ }^{38}$ is similar to that discussed here for pentacene.

The solution of the coupled differential equations of motion (10) for the elements of the density matrix must be found to interpret the coherent transients one sees in the laboratory. Effects due to inhomogeneous broadening and the nonuniform spatial distribution in $\epsilon$ of the laser must also be treated. We shall deal with each transient separately and focus our attention only upon those that we have observed in pentacene.

\section{Optical nutation}

Optical nutation has been observed recently in solids by Orlowski and Zewail. ${ }^{25}$ To describe these nutation signals one must determine the time dependence of the off-diagonal elements of the density matrix because these elements of $\rho$ are related to the polarization $P$ (amplitude $\vec{P}$ ) induced by the laser in an ensemble density of $N$ molecules according to the following expres sion:

$$
\begin{aligned}
P(t) & =N \mu\left(\rho_{0 p} e^{+i \omega t}+\rho_{00} e^{-i \omega t}\right) \\
& =N \mu\left(r_{1} \cos \omega t-r_{2} \sin \omega t\right),
\end{aligned}
$$

where from the FVH picture:

$$
\left(\begin{array}{c}
r_{1} \\
r_{2} \\
r_{3}
\end{array}\right)=\left(\begin{array}{c}
\rho_{0 p}+\rho_{p 0} \\
i\left(\rho_{p 0}-\rho_{0 p}\right) \\
\rho_{p p}-\rho_{00}
\end{array}\right) \text {. }
$$

Thus the polarization acts as a source term in Maxwell's equation and provides a field amplitude $\epsilon_{s}$ in the solid (optically thin) that is given by

$$
\epsilon_{s} \simeq-i \frac{\omega L \eta}{2 c \epsilon_{0}} \bar{P},
$$

where $L$ is the crystal thickness, $\epsilon_{0}$ the background dielectric constant, $\eta$ the corresponding refractive index, and $c$ the speed of light. Since the macroscopic polarization is given by $r_{1}$ and $r_{2}$ averaged over the inhomogeneous line shape, and because $r_{1}$ is an odd function of $\Delta$ while $r_{2}$ is an even function of $\Delta$, we can write $\epsilon$, as follows [see Eq. (12)]:

$$
\epsilon_{8}=\frac{\omega L \eta}{2 c \epsilon_{0}} N \mu\left\langle r_{2}\right\rangle=\frac{i \omega L \eta}{c \epsilon_{0}} N \mu\left\langle\rho_{p 0}\right\rangle .
$$

Therefore, to obtain $\left\langle r_{2}\right\rangle$ or $\left\langle\rho_{p 0}\right\rangle$, Eq. (10) must be solved. Using Laplace transformation techniques, Schenzle and Brewer ${ }^{39}$ solved Eq. (10) and we show their result for $r_{2}$ in the high power limit (i.e., $\chi \gg 1 / T_{1}, 1$ ) $T_{2}$ ) which applies in our case:

$$
r_{2}=\chi \exp \left[-\left(\frac{1}{T_{2}}+\frac{1}{2 T} \frac{\chi^{2}}{\chi_{1}^{2}}\right) t\right] \frac{\sin \chi_{1} t}{\chi_{1}} .
$$

Here $1 / T=1 / T_{1 p 0}-1 / T_{2}$, and $\chi_{1}^{2}=\Delta^{2}+\chi^{2}$.

Eq. (15) must now be averaged over the inhomogeneous line shape $g(\omega)$ of the solid. We assume a Gaussian line shape function (normalized area) of the form:

$$
g(\Delta)=\frac{1}{\sqrt{\pi} \delta \omega_{I}} \exp \left(-\Delta^{2} / \delta \omega_{l}^{2}\right),
$$

where $\delta \omega_{I}$ is the width of the inhomogeneous line centered at $\omega=\omega_{0}$.

For pentacene and for many other solids in the optical region $\chi \ll \delta \omega_{1}$, and therefore the averaged value of $r_{2}$ can be written in the following way (see Appendix $B$ ):

$$
\left.\left\langle r_{2}(t)\right\rangle \simeq \chi e^{-t / T_{2}}\left\langle\exp \left[\frac{-1}{2 T}\left(\frac{\chi^{2}}{\Delta^{2}}\right) t\right]\right\rangle \frac{\sin \chi_{1} t}{\chi_{1}}\right\rangle .
$$

This factorization of the averaging is valid especially at short times and has been checked numerically. ${ }^{39}$ The first integral can be evaluated analytically while the second integral has been approximated by the usual Bessel function description for optical nutation ${ }^{16}$; i. e.,

$$
\left\langle r_{2}(t)\right\rangle \simeq \chi \frac{\sqrt{\pi}}{\delta \omega_{1}} \exp \left[-\frac{t}{T_{2}}-\frac{\chi}{\delta \omega_{I}}\left(\frac{2 t}{T}\right)^{1 / 2}\right] J_{0}(\chi t) .
$$

This solution is valid except near $t=0$ because of the approximations made to evaluate the integrals. Later we will correct Eq. (18) so that it will be valid for all times. Now, since $\delta \omega_{I} \gg \chi$ we can simplify Eq. (18) even further:

$$
\left\langle r_{2}(t)\right\rangle \simeq \chi \frac{\sqrt{\pi}}{\delta \omega_{t}} J_{0}(\chi t) e^{-t / T_{2}}
$$

To complete our calculation we must average Eq. (19) over the measured transverse profile of the $T E \mathrm{M}_{00}$ Gaussian beam of our laser (Fig. 3) since individual molecules will see different field strengths along the width of the beam and will therefore have different $\chi$ 's. This will smear out the Bessel function causing more rapid damping of the transient.

First, let us consider the signal at the detector. As a square-law device, it will see an intensity $I(t)$ given by:

$$
I(t)=c \epsilon_{0}\left\langle\left[\epsilon \cos \omega t+\epsilon_{s} \cos \omega t\right]^{2}\right\rangle_{t} .
$$

The time average simply yields

$$
I(t)=c \epsilon_{0}\left(\frac{\epsilon^{2}}{2}+\epsilon_{3} \epsilon+\frac{\epsilon_{3}^{2}}{2}\right) .
$$

We may assume for optically thin samples that $\epsilon_{\mathrm{s}} \ll \epsilon$. Defining $I_{0}=\frac{1}{2} c \epsilon_{0} \epsilon^{2}$ the total signal at any time $t$ is: 


$$
I(t)=I_{0}+c \epsilon_{0} \epsilon \epsilon_{s}(t) .
$$

Equation (22) reveals the amplification of the signal field by the laser field, similar to the heterodyne detection scheme. Combining Eqs. (14), (19), and (22) we have:

$$
I(t)=I_{0}-A \chi^{2} J_{0}(\chi t) e^{-t / T_{2}},
$$

where $A$ is a constant: $A=(\sqrt{\pi} / 2) \hbar \omega L N / \delta \omega_{I}$ and $\eta=1$.

If we replace $\epsilon$ by $\epsilon e^{-R^{2} / 2 B_{L}}{ }^{2}$, where $B_{L}$ is the laser beam half-width $(1 / e)$ of the intensity profile (not field), and integrate over the surface area of the detector, which is larger than the beam diameter, our total signal is:

$$
I_{T}(t)=2 \pi \int_{0}^{\infty} I(t) R d R .
$$

If we make the substitution

$$
\chi^{\prime}=\chi t \exp \left[-R^{2} /\left(2 B_{L}^{2}\right)\right]
$$

Eq. (24) can be solved easily to yield

$$
\begin{aligned}
I_{T}(t) & =\pi B_{L}^{2}\left\{I_{0}-\frac{2 A}{t^{2}} e^{-t / T_{2}} \int_{0}^{x t} \chi^{\prime} J_{0}\left(\chi^{\prime}\right) d \chi^{\prime}\right\} \\
& =\pi B_{L}^{2}\left\{I_{0}-2 A\left(\frac{\chi}{t}\right) J_{1}(\chi t) e^{-t / T_{2}}\right\} .
\end{aligned}
$$

Shoemaker and Van Stryland ${ }^{23}$ obtained results similar to Eq. (26) when dealing with gases in the IR region, except for the exponential term containing $T_{2}$. In their case they assumed that the long $T_{1}=T_{2}$ (which is reasonable) and thus the nutation essentially damps by the preexponential terms of Eq. (26). However, in our case (optical) $T_{2}$ is short enough so that the transient decay is given by the full expression (26). Because of this the nutation signal when treated properly can give $T_{2}$. We see that averaging over the laser beam causes the transient to damp faster than the result of Eq. (23) which describes only the inhomogeneous averaging. However, the oscillation frequency is essentially unchanged. This point is illustrated with the computer simulation of Eqs. (23) and (26) shown in Fig. 5, and will be clearly demonstrated when we fit the nutation data of pentacene.

Although $J_{1}(\chi t)$ goes to zero at $t=0$, the factor of $1 / t$ in the expression for the nutation obtained by averaging over the laser beam causes Eq. (26) to be invalid near $t=0(\chi t<1)$. We can correct this behavior with an error function similar to that used by Tang and Silverman ${ }^{18}$ in their discussion of the nutation effect. Since the limiting form of $J_{1}(\chi t)$ for small arguments is:

$$
\left.J_{1}(\chi t)\right|_{\text {short t1 mea }} \approx \frac{\chi^{t}}{4},
$$

an error function of the form:

$$
\operatorname{erf}\left(\frac{x t}{2}\right)=\frac{2}{\sqrt{\pi}} \int_{0}^{x t / 2} e^{-\alpha^{2}} d \alpha
$$

will correct the behavior of Eq. (26) and provide a result valid for all $t$, within the framework of the above mentioned approximations. Thus we have

$$
I_{T}(t)=\pi B_{L}^{2}\left\{I_{0}-2 A\left(\frac{\chi}{t}\right) \operatorname{erf}\left(\frac{\chi t}{2}\right) J_{1}(\chi t) e^{-t / T_{2}}\right\} .
$$

This result (a) reveals the importance of averaging over the laser beam if one is interested in relating the observed decay (see Fig. 5) of the transient to $T_{2}$ and (b) shows that an accurate value of the transition dipole moment can be obtained by fitting the observed transient since the oscillation of the signal is still given by the Rabi frequency $x$.

\section{Incoherent resonance decay}

The initial jump in emission intensity seen on the IRD for pentacene is due to the coherent driving of a new group of molecules ( $\beta$ of Fig. 1) brought into resonance with the switched laser frequency. For short times, we may use the solution of Eq. (10b) to predict the jump in emission intensity and from it characterize the temperature dependence of the IRD obtained experimentally.

We will present the complete solution for $\rho_{p p}(t)$ obtained using Laplace transform techniques and the perturbative expansion approach used by Schenzle and Brewer. ${ }^{39}$ This solution has not been presented before and is generally applicable for solids at low temperature.

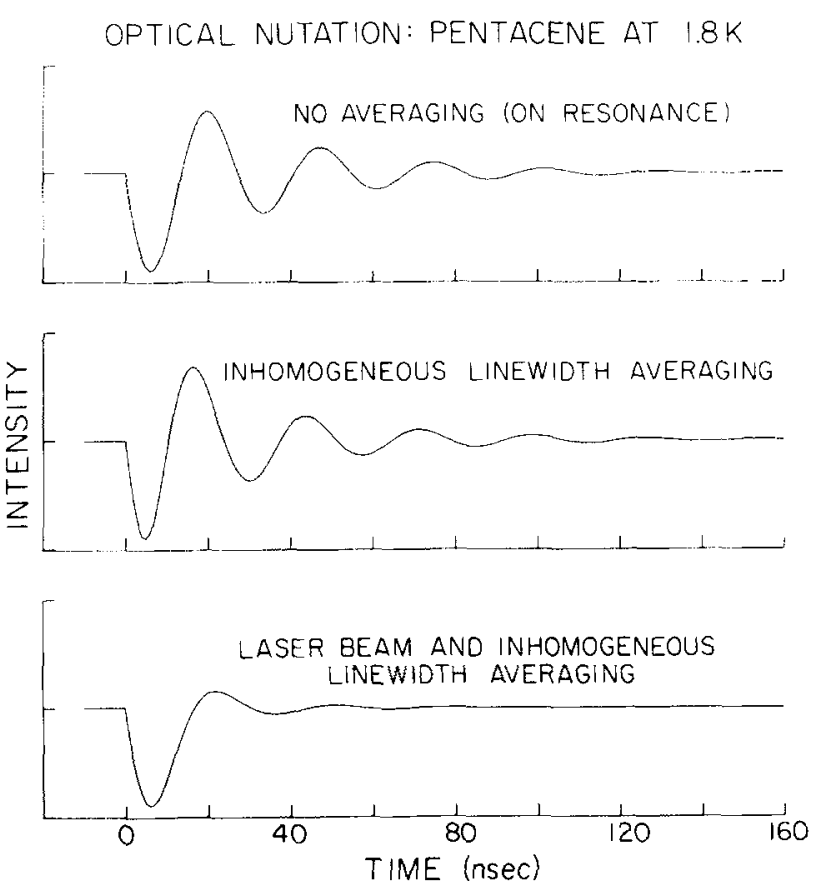

FIG. 5. Shown at the top is a plot of the expression [Eq. (15)] for the optical nutation in pentacene without averaging (on resonance case). In the middle is shown the result of averaging over the inhomogeneous linewidth [Eq. (19)]. Here Eq. (19) is multiplied by the error function shown in Eq. (28) for proper behavior at $t=0$. Finally, at the bottom, is shown a plot of Eq. (29) which considers averaging over both the inhomogeneous lineshape and the laser beam spatial profile. In all three graphs, the Rabi frequency $(36.6 \pm 0.6 \mathrm{MHz})$ is the same; only the amplitude and transient decay time are affected by the averaging process. Results obtained in Fig. 3 were used in the averaging over the laser beam. 


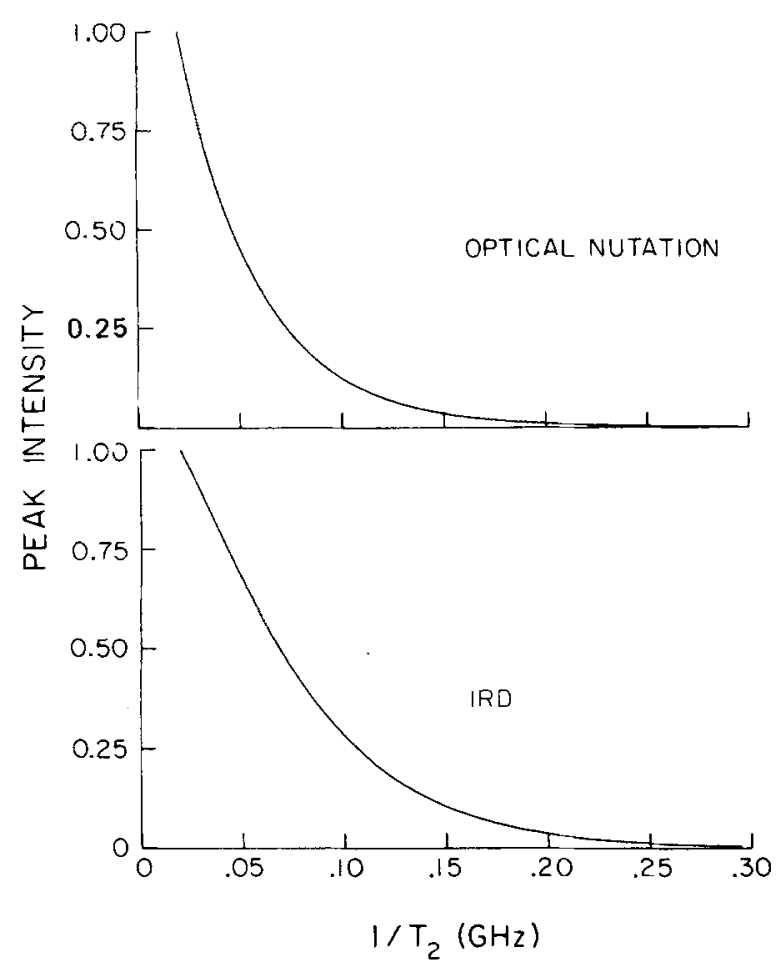

FIG. 6. The dependence of the (relative) optical nutation and IRD peak amplitudes on $T_{2}$ from Eqs. (29) and (32), respectively. In the nutation expression the dc term $\pi B_{L}^{2} I_{0}$ is ignored. In the IRD expression, a factor of $T_{2}^{-1}$ appears in the preexponential factor which causes the amplitude to "decay" slower than the nutation.

Using Eq. (10b) and solving for the diagonal elements of $\rho$ we have:

$$
\begin{aligned}
\rho_{p p}(t)= & \frac{-\frac{1}{2} \chi^{2}}{\left(\lambda_{1}-\lambda_{2}\right)^{2}}\left\{\left(1+\frac{1}{\lambda_{1} T_{2}}\right) e^{\lambda_{1} t}\right. \\
& \left.+e^{\lambda_{2} t}\left(A \cos \chi_{1} t+\frac{B \sin \chi_{1} t}{\chi_{1}}\right)\right\}+\frac{1}{2} \frac{\chi^{2}}{T_{2} \lambda_{1}\left(\lambda_{2}^{2}+\chi_{1}^{2}\right)},
\end{aligned}
$$

where

$$
\begin{aligned}
& \lambda_{1}=\frac{-1}{T_{1 p 0}}+\frac{\chi^{2} T}{1+T^{2} \chi_{1}^{2}} \\
& \lambda_{2}=-\frac{1}{T_{2}}-\frac{\chi^{2} T}{2\left[1+T^{2} \chi_{1}^{2}\right]} \\
& A=\frac{\left(\lambda_{1}-2 \lambda_{2}\right)}{T_{2}\left(\lambda_{2}^{2}+\chi_{1}^{2}\right)}-1
\end{aligned}
$$

and

$$
B=\left(\lambda_{2}-\lambda_{1}\right)+\frac{\lambda_{2}\left(\lambda_{2}-\lambda_{1}\right)-\chi_{1}^{2}}{T_{2}\left(\lambda_{2}^{2}+\chi_{1}^{2}\right)} .
$$

We should emphasize that the above solution is for the level structure described earlier for pentacene. In the case where the $T_{1}$ 's of the excited and ground states are equal the solution is not the same as (30a) but yields identical results to Eq. (5) of Ref. 27.

Now, Eq. (30) represents the time dependence of the emission intensity for the group of molecules on resonance with the laser. Off-resonance molecules provide the transient decay. For the initial jump in emission intensity which relates to $T_{2}$ we will ignore the contribution from the decay of the group switched off-resonance. This is equivalent to saying that we are in the high power limit where $\chi \gg 1 / T_{1}, 1 / T_{2}$. The oscillations predicted by Eq. (30) have been observed experimentally ${ }^{40}$ but due to the drastic smoothing that takes place when averaging Eq. (30) over the laser beam intensity profile and the inhomogeneous linewidth, one will not observe them easily without subtracting the background resulting from the off-resonance decaying molecules.

In the high power limit and at short times, Eq. (30) reduces to:

$$
\rho_{p p}(t) \simeq-\frac{1}{2} e^{x_{2} t} B \sin \frac{\chi_{1} t}{\chi_{1}},
$$

where $B$ is now $\approx-\frac{1}{2}\left(1 / T_{1 p 0}+1 / T_{2}\right)$ and the steady state term has been ignored. Thus, we recover a function very similar to that found for the nutation [Eq. (15)]. Averaging (31) over the inhomogeneous linewidth and making the previous approximation (i.e., $\chi \ll \delta \omega_{l}$ ) we obtain the solution:

$$
\left\langle\rho_{\rho p}(t)\right\rangle=\frac{1}{4}\left(\frac{1}{T_{1 p 0}}+\frac{1}{T_{2}}\right) \frac{\sqrt{\pi}}{\delta \omega_{I}} J_{0}(\chi t) e^{-t / T_{2}} .
$$

As we shall see later, this result will be valid for examining the temperature dependence of the initial jump in the IRD. If we measure the relative amplitude of Eq. (32) at fixed $t$ near the peak for various values of $T_{2}$ (see Fig. 6), averaging over the laser beam (which introduces a faster damping rate and decreased absolute intensity) is unnecessary since we are making a relative intensity measurement at fixed $t$ when examining this $T_{2}$ dependence in Eq. (32). Note that to obtain the "exact" functional form for $\left\langle\rho_{p p}(t)\right\rangle$ we must follow the procedure done in the nutation section.

\section{Optical free induction decay}

When a group of molecules initially on resonance (under steady-state conditions) is allowed to freely radiate by rapidly switching the frequency of the laser off-resonance, the solution for the off-diagonal density matrix element (and thus the polarization) is from Eq. (10) with $\chi=0$ :

$$
\rho_{0 p}(t)=\rho_{0 p}(\infty) \exp \left[\left(-1 / T_{2}+i \Delta\right) t\right] .
$$

For the pentacene case we obtained the following result:

$$
\rho_{0 p}(t)=-\frac{\chi}{2} \rho_{00}(0) \frac{\left(\frac{i}{T_{2}}-\Delta\right) e^{\left(-1 / T_{2}+i \Delta\right) t}}{\Delta^{2}+\left[\left(\frac{1}{T_{2}}\right)^{2}+\frac{\chi}{2} \frac{T_{100}}{T_{2}}\right]} .
$$

Averaging this result over the inhomogeneous linewidth one has: ${ }^{17,39}$

$$
\left\langle\rho_{0 p}(t)\right\rangle \simeq \frac{i \chi \sqrt{\pi}}{2 \delta \omega_{I}}\left(1-\left\{\frac{\frac{1}{T_{2}}}{\left[\left(\frac{1}{T_{2}}\right)^{2}+\frac{\chi^{2}}{2} \frac{T_{100}}{T_{2}}\right]^{1 / 2}}\right\}\right)
$$




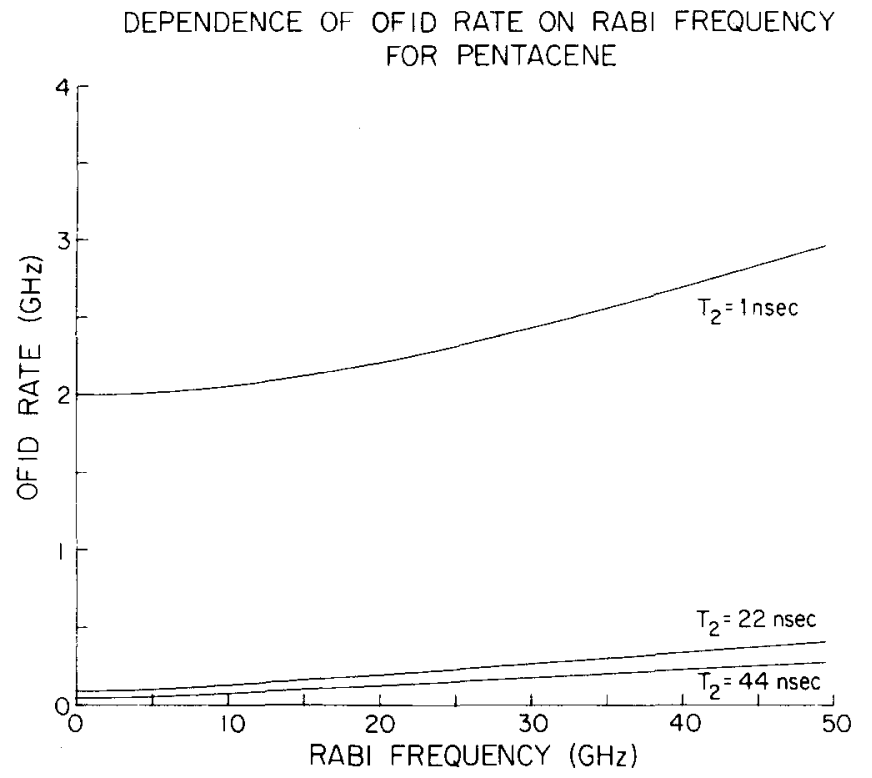

FIG. 7. Shown here are graphs of the OFID rate dependence on the Rabi frequency in pentacene for various values of $T_{2}$. $T_{1}$ has been fixed at $23.5 \mathrm{~ns}$. Extrapolated to $\chi=0$, these plots allow one to obtain $T_{2}$ (intercept $=T_{2} / 2$ ) for the optical transition. As shown in the graph, the power-broadening term in Eq. (36) dominates the expression at high laser power and the observed decay becomes linear in $\chi$.

$$
\times \exp \left\{-\left[\frac{1}{T_{2}}+\left(\frac{1}{T_{2}^{2}}+\frac{\chi^{2}}{2} \frac{T_{180}}{T_{2}}\right)^{1 / 2}\right] t\right\} .
$$

To obtain the signal field $\epsilon_{s}$ we use Eq. (14) and (35) $(\eta=1)$ :

$$
\begin{aligned}
\epsilon_{s}(t) \simeq & \frac{\omega L N \mu}{c} \frac{\chi^{\sqrt{\pi}}}{2 \delta \omega_{I}}\left(1-\frac{1}{\left[1+\frac{\chi^{2}}{2} T_{1 p 0} T_{2}\right]^{1 / 2}}\right)^{\prime} \\
& \times \exp \left\{-\left[\frac{1}{T_{2}}+\left(\frac{1}{T_{2}^{2}}+\frac{\chi^{2}}{2} \frac{T_{1 p 0}}{T_{2}}\right)^{1 / 2}\right] t\right\} .
\end{aligned}
$$

In the FID experiment the signal at the detector [different from 20] is:

$$
I_{T}(t)=c \epsilon_{0}\left\langle\left[\epsilon \cos \omega t+\epsilon_{s} \cos \omega_{s} t\right]^{2}\right\rangle_{t}
$$

which for optically thin samples, ignoring the high frequency $\left(\omega+\omega_{s}\right)$ component, is

$$
I(t)=I_{0}+2 c \epsilon_{0} \epsilon \epsilon_{s}(t) \cos \left(\omega-\omega_{s}\right) t .
$$

Thus, one should see heterodyne beating at $\left(\omega-\omega_{s}\right)$ superimposed on a power dependent exponential decay. As one can see from Eq. (36), in the low power regime (i.e., $\chi \ll 1 / T_{1}, 1 / T_{2}$ ) the FID gives $T_{2}$ directly, and this is verified from our pentacene results. The dependence of the decay rate on $\chi$ is depicted in Fig. 7 . It should be mentioned that the above treatment is only valid for the case where the population remains in the two level system. If, however, the system is statistically open with net population loss then the treatment of Jones et al. ${ }^{41}$ must be used. The closed two-level system theory describes our pentacene solid state results quite well.

\section{B. Steady-state coherence regime: application of Wilcox-Lamb method to pentacene}

In this limit, $\tau_{0}$, the observed transient decay time is much longer than $(\hbar / \mu \cdot \epsilon)$ and $T_{2}$. Thus, if $\tau_{0} \sim 10$ $\mu \mathrm{s}$ and $T_{2}$ is in the nanosecond range as in pentacene, we can assume that transient dephasing is complete and that the off-diagonal density matrix elements connecting levels $|0\rangle$ and $|p\rangle$ have decayed to their steady-state values in the presence of the laser field.

We may smoothly enter this regime using the WilcoxLamb method ${ }^{42}$ for deriving density matrix equations from the original Bloch equations. This method has been used to show in several cases ${ }^{43}$ that rate equations yield solutions equivalent to the time-averaged (i.e., the usual transient solution "without" coherent oscillations) Bloch solutions for describing population flow in multilevel systems.

For the pentacene case, we shall first modify Eq. (10) to include relaxation terms to and from the $\{|l\rangle\}$ manifold, and then provide analytical solutions for the EO and LADS experiments which involve entirely different descriptions. With this in mind, Eq. (10) can now take the form:

$$
\begin{aligned}
& \dot{\rho}_{00}=\Gamma_{p 0} \rho_{p p}+\Gamma_{l 0} \rho_{u l}+\frac{1}{2} i \chi\left(\rho_{p 0}-\rho_{0 p}\right) \\
& \dot{\rho}_{p p}=-\left(\Gamma_{p 0}+\Gamma_{p l}\right) \rho_{p p}+\frac{1}{2} i \chi\left(\rho_{0 p}-\rho_{p 0}\right) \\
& \dot{\rho}_{l l}=\Gamma_{p l} \rho_{p p}-\Gamma_{t 0} \rho_{u} \\
& \dot{\rho}_{0 p}=(-\Gamma+i \Delta) \rho_{0 p}+\frac{1}{2} i \chi\left(\rho_{p p}-\rho_{00}\right) .
\end{aligned}
$$

We note that the structure of $\{|l\rangle\}$ is unspecified and therefore the $\Gamma$ 's which describe the feeding into and the decay from $l$ represent an average quantity. We will come back later to this point. Now, the first step in the Wilcox-Lamb method involves setting the time. derivatives of the off-diagonal density matrix elements to zero. This step is based on the condition that the off-diagonal elements dephase quickly to their steady state values. Expressing our result for Eq. (39d) in terms of $r_{2}$ and $r_{3}$ [see Eq. (13)] we obtain:

$$
r_{2}=\left(\frac{\chi \Gamma}{\Gamma^{2}+\Delta^{2}}\right) r_{3} \text {. }
$$

Substituting (40) into the differential equations for the diagonal density matrix elements of (39) and assuming a statistically closed system (i. e., $\rho_{00}+\rho_{p p}+\rho_{l l}=1$ ) we obtain the following rate equations for the on-resonance case:

$$
\begin{aligned}
& \dot{\rho}_{p p}+\left(\Gamma_{p 0}+\Gamma_{p l}+\frac{1}{2} \chi^{2} T_{2}\right) \rho_{p p}-\frac{1}{2} \chi^{2} T_{2} \rho_{00}=0 \\
& \dot{\rho}_{00}+\left(\frac{1}{2} \chi^{2} T_{2}+\Gamma_{10}\right) \rho_{00}-\left(\frac{1}{2} \chi^{2} T_{2}+\Gamma_{p 0}-\Gamma_{l 0}\right) \rho_{p p}=\Gamma_{l 0} .
\end{aligned}
$$

We now consider the solution appropriate for the two different experiments (EO and LADS) done on pentacene, and consider off-resonance effects later.

\section{LADS experiments}

We can solve the above set of differential equations analytically using Laplace transform methods and Cramer's Rule. The solution presented here for $\rho_{p p}$, pro- 
vides us with the parameter directly related to our experimental observable: emission intensity. Under the above mentioned conditions, and provided that the laser is turned on at $t=0$ (i.e., $\chi=0$ when $t<0$ ), the solution is given by:

$$
\begin{aligned}
\rho_{p p}(t)= & \frac{R_{p}}{\lambda_{+}-\lambda_{-}} \\
& \times\left[\left\{\frac{\Gamma_{10}+\rho_{00}(0) \lambda_{+}}{\lambda_{+}}\right\} e^{\lambda_{+} t-}\left\{\frac{\Gamma_{10}+\rho_{00}(0) \lambda_{-}}{\lambda_{-}}\right\} e^{\lambda_{-} t}\right]+\frac{R_{p}}{\lambda_{+} \lambda_{-}} \Gamma_{10}
\end{aligned}
$$

Here $\rho_{00}(0)$ is the population of $|0\rangle$ at $t=0$, and $R_{p}=\frac{1}{2} \chi^{2} T_{2}$. For most experiments we set $\rho_{00}(0)=1$, although the extension for other cases (e.g., when describing $\rho_{00}$ at the end of short laser pulses) where $\rho_{00}(0) \neq 1$ is straightforward and will be discussed later. The arguments of the exponentials, $\lambda_{ \pm}$, satisfy the following quadratic equation:

$$
\begin{aligned}
2 \lambda_{ \pm}= & -\left(2 R_{p}+\Gamma_{10}+\Gamma_{p 0}+\Gamma_{p l}\right) \\
& \pm\left[\left(2 R_{p}-\Gamma_{10}+\Gamma_{p 0}+\Gamma_{p l}\right)^{2}-4 R_{p} \Gamma_{p l}\right]^{1 / 2} .
\end{aligned}
$$

Equation (43) provides a function that rapidly builds up at $t=0$ to a peak value $\left(t=t_{p}\right)$ and then decays by essentially a single exponential $\left(e^{\lambda_{-}} \approx 0\right.$ for $\left.t>t_{p}\right)$ to an equili brium value $\rho_{p p}\left(t_{o q}\right)$. The decay time $\tau_{0}$ and the equilibrium value are determined by $\Gamma_{p l}$ and $\Gamma_{l 0}$. In our experiments the laser does not turn on instantly so we have modified the pumping rate in Eq. (43) to account for the finite rise time $\Gamma_{L}^{-1}$ of our laser pulse. To do this we simply replace $R_{p}$ by

$$
R_{p}(t)=\left(1-e^{-\Gamma_{L} t}\right) R_{p}(\max )
$$

EMISSION INTENSITY VS. TIME FOR STEADY-STATE COHERENCE IN PENTACENE LEVEL STRUCTURE

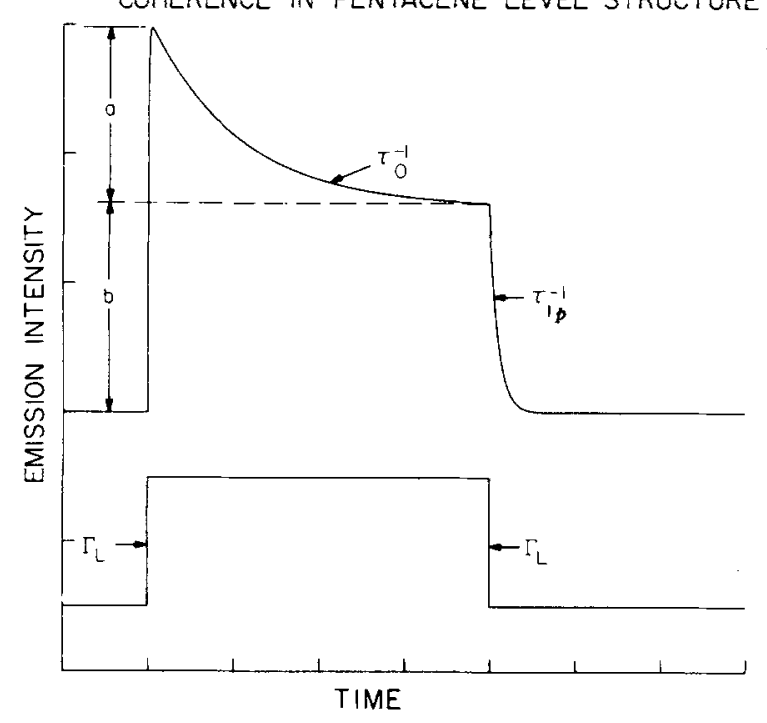

FIG. 8. Plotted here are the theoretical transients obtained from Eqs. (43) and (46) for describing LADS emission experiments (top) and the excitation pulse (bottom). The slow decay during the pulse is labelled $\tau_{0}^{-1}$ and the fast decay at the falling edge of the pulse is $\tau_{1 \phi}^{-1}$, the observed emission rate of the prepared state $|p\rangle$ convoluted by the pulse decay, $\Gamma_{L}$. The quantity, $a$, is the magnitude of the "overshoot" in emission intensity observed before reaching a final steady-state value, $b$. The ratio $\gamma=a / b$ will be referred to in later figures. where $R_{p}(\max )$ is the maximum value for the pumping rate, and the laser pulse has been given an exponential buildup which was measured and verified experimentally. Equation (43) gives the population of the emitting level as a function of time when the laser is on-resonance. The off-resonance solution (i.e., $R_{p}=0 ; t>t^{\prime}$ ) for $\rho_{p p}$ can be written as

$$
\rho_{p p}\left(t>t^{\prime}\right)=\rho_{p p}\left(t=t^{\prime}\right) e^{-t / T_{1 p}} .
$$

Here, $t^{\prime}$ is the time at which the laser pulse ends and so Eq. (46) is simply an exponential decay of population from the primary level. The initial value $\rho_{p p}\left(t^{\prime}\right)$ equals the equilibrium value on resonance if the laser pulse width is long enough.

Using Eqs. (43)-(46) one can generate the curve shown in Fig. 8 which, as we shall show later, fits our results quite well. In the same figure the pulse used in the excitation into $|p\rangle$ is also depicted.

Physically, the model can be explained in the following manner. As the population builds up in $|p\rangle$ some of it gets trapped in the slow decay channel involving $|l\rangle$ before returning to $|0\rangle$. Following the fast initial buildup, population is redistributed until $\rho_{p p} \Gamma_{p l}$ and $\rho_{l l} \Gamma_{l 0}$ are equal and equilibrium results.

Two quantities labeled $a$ and $b$ in Fig. 8 play different roles. The first, $a$ is the extent to which $\rho_{p p}$ is driven beyond equilibrium; it increases as $\Gamma_{p l}$ increases and gives a measure of how much of the total populations is "tied up" in the slow decay channel. For long pulses, the second parameter, $b$, is the equilibrium population of $|p\rangle$. The ratio $\gamma=a / b$ is therefore determined by the ratio $\Gamma_{p l} / \Gamma_{10}$ for fixed $R_{p}$. If one simultaneously fixes $\tau_{0}$, the observed decay time, then a unique solution exists because only one value of $\Gamma_{10}$ and the ratio $\Gamma_{p 1} / \Gamma_{10}$ can provide both the observed $\gamma$ and $\tau_{0}$. The interrelationship of the parameters and the physical significance of certain trends can be more easily understood by examining Figs. 9 and 10. Here $\Gamma_{10}$, is fixed as we examine (1) the ratio $(\gamma)$ vs $\Gamma_{p l} / \Gamma_{t 0}$ at various pumping rates $R_{p}$ in Fig. 9; (2) the observed decay time $\tau_{0}$ vs $\Gamma_{p l} / \Gamma_{l 0}$ in Fig. 9, and finally (3) the observed decay time $\tau_{0}$ vs $R_{p}$ in Fig. 10. From Fig. 9 we see that $\gamma$ is directly proportional to the ratio $\Gamma_{p l} / \Gamma_{l 0}$. Physically, this means that more population is trapped in the slow decay channel as $\Gamma_{p l}$ increases (with $\Gamma_{l 0}$ fixed). If the pumping rate increases, more population should be trapped for any given $\Gamma_{p l} / \Gamma_{t 0}$ since more population is available in $|p\rangle$. This effect shows up as a change in the slope of the lines of Fig. 9 (bottom). Also, the intercept of this plot is zero which it should be since if the slow decay channel is removed $\left(\Gamma_{p l}=0\right), \rho_{p p}$ should approach its equilibrium value $(b)$ with no overshoot. Finally, for fixed $\gamma$, the observed $\tau_{0}$ is constant on this plot (i.e., a horizontal line represents a constant $\tau_{0}$ decay time). Thus, for a given $\tau_{0}$ there are different ratios of $\Gamma_{p l} / \Gamma_{l 0}$ (corresponding to different pumping rates) that provide identical $\gamma$ values.

Figure 9 (top) shows a plot of observed decay time $\tau_{0}$ vs $\Gamma_{p l} / \Gamma_{b 0}$. As expected, the decay time increases as $\Gamma_{p l}$ goes to zero. Careful examination of Fig. 9 indicates that there is only one value of $\Gamma_{p l}$ and $\Gamma_{10}$ that will 


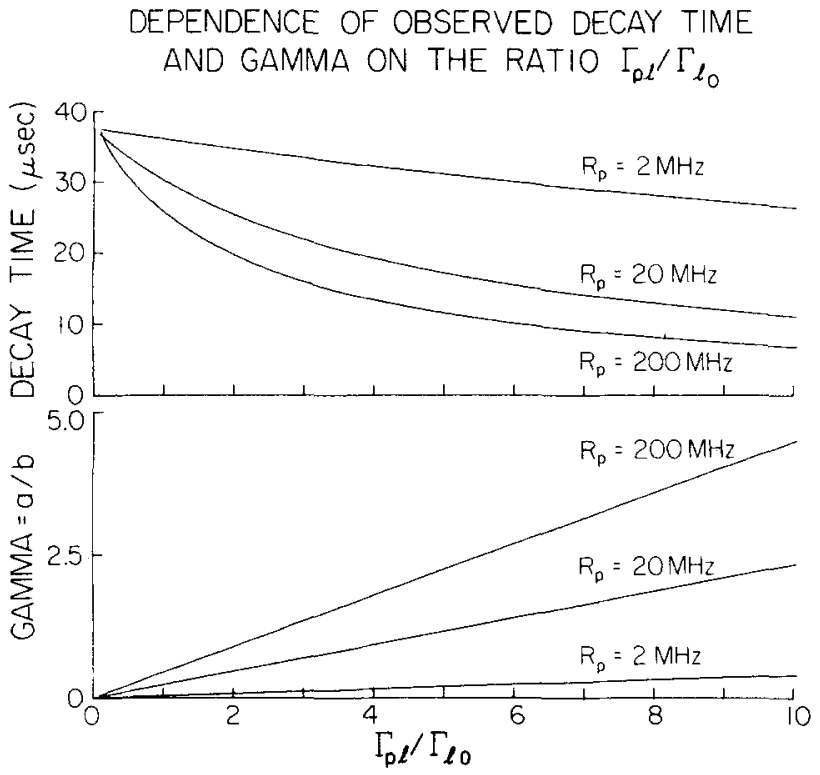

FIG. 9. The dependence of the slow transient decay time, $\tau_{0}$, and $\gamma=a / b$ on the ratio $\Gamma_{p y} / \Gamma_{10}$ [from Eq. (43)]. In these graphs, $\Gamma_{10}$ is fixed at the best fit value $(0.0266 \pm 0.002 \mathrm{MHz})$ obtained from the EO experiments, $\Gamma_{p 0}=40 \mathrm{MHz}$ and $R_{p}=\frac{1}{2} \chi^{2} T_{2}$ is the pumping rate in the steady-state coherence regime. As the ratio $\Gamma_{p l} / \Gamma_{l 0}$ decreases, both the decay rate, $\tau_{0}^{-1}$, and $\gamma$ decrease. Thus, a measurement of the decay time at very small values of $\Gamma_{p l} / \Gamma_{l 0}$ is difficult to make accurately.

simultaneously provide (1) the observed decay time and (2) the observed $\gamma$ for fixed $R_{p}$.

Figure 10 depicts a plot of the observed decay time $\tau_{0}$ vs $R_{p}$ for various ratios of $\Gamma_{p l} / \Gamma_{10}$. This plot as well as Fig. 9 show how insensitive the model is to the choice of $R_{\phi}$, especially in the high power limit. In addition, one sees that the observed decay time increases as $R_{p}$ decreases. This is understandable physically since as the pumping rate decreases it must take longer to achieve equilibrium.

\section{EO experiments}

For these experiments, the treatment is somewhat more complicated than that described above for LADS, since in EO experiments the laser is switched into resonance with another ensemble instead of being turned oif completely. Thus one must keep track of the time-dependent emission characteristics (population) of both groups simultaneously. At the beginning of an EO experiment $(t<0)$ the laser is on-resonance with one group ( $\alpha$ of Fig. 1) and this group has reached its steady state value $\rho_{p p}^{\langle\alpha\rangle}\left(t_{o q}\right)$. At $t=0$ the laser is switched into resonance with a new group, $\beta$. Equation (43) can be used to determine the buildup of population $\rho_{p p}$ due to group $\beta$ while the off-resonance solution, Eq. (46), will provide the decrease in population due to the decay of $\alpha$ molecules. The sum of the two provides the total population $\rho_{p p}^{\alpha}+\rho_{p p}^{\beta}$ and hence the total emission signal. Now, the buildup population rate from $\beta$ does not usually equal the decay rate from $\alpha$. Thus, an "overshoot" is expected if the $\beta$ buildup is faster than the $\alpha$ decay and an undershoot for the reverse condi- tions. Whether or not this phenomenon is observed experimentally will be determined by $R_{p}, T_{1 p}$ and, of course, detector response time and the signal averager time resolutions. We have observed an overshoot in pentacene (i. e., $\beta$ buildup $>\alpha$ decay) using a fast photomultiplier $(1 \mathrm{GHz})$ in connection with a sampling scope.

To complete the analysis of the EO experiments we must consider what happens when the laser is switched back to the original frequency. Population in $\beta$ will decay from the value at $t=t^{\prime}$ (the switching time) according to Eq. (46) while population in $\alpha, \rho_{p p}^{\alpha}$, will buildup again according to Eq. (43). If the frequency-switching pulse is long compared to $\tau_{0}$, the total population will evolve in time (beginning at $t^{\prime}$ ) just as it did at $t=0$. This is shown in Fig. 11 for various values of the pumping rate, $R_{p}$. However, if the pulse is shorter than or comparable with $\tau_{0}$, all of the population has not yet returned to $|0\rangle$ for the $\alpha$ group at $t=t^{\prime}$. Thus, the buildup will not be as large as it is for the long pulses and the net population will decrease at a rate determined by $\left(1 / T_{1 p}\right)$. To more fully explain this we must go back to Eq. (43) and realize that $\rho_{00}(0)$ is the population of the ground state when the system is brought into resonance. The boundary condition for $\rho_{00}(0)$ is usually 1 which means the group was initially off-resonance for a long time compared to $\tau_{0}$ such that all population had adequate time to return to $|0\rangle$. For short switching pulses we need to calculate the off-resonance solution for $\rho_{00}(t)$ and use it to determine the proper initial value of $\rho_{00}\left(t^{*}\right)$ in Eq. (43).

The solution is found by solving the same differential

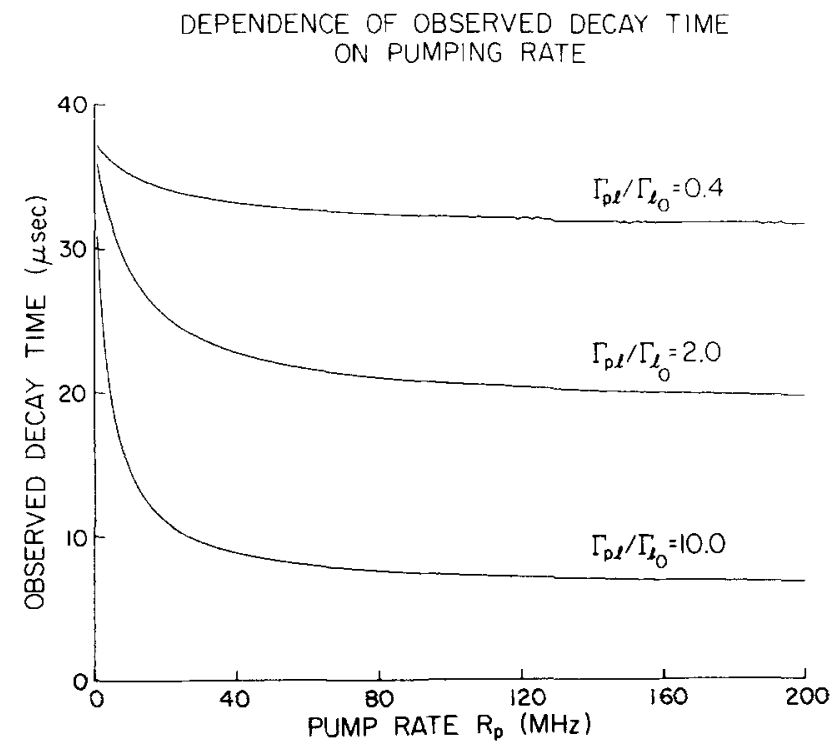

FIG. 10. Shown here is the dependence of $\tau_{0}$ on the pumping rate, $R_{\rho}$ obtained from Eq. (43) for various ratios of $\Gamma_{p l} / \Gamma_{l 0}$. As in Fig. 9, $\Gamma_{20}$ and $\Gamma_{p 0}$ are fixed. At very low pumping rates, the decay time increases but since the overshoot, $a$, becomes very small also (see Fig. 9), $r_{\theta}$ cannot easily be measured experimentally. Mast of the experiments in pentacene were carried out in the flat portion of the curves. Note that at these higher power densities absorption from $p$ to higher excited states is feasible, although fast relaxation from these states will hinder such processes. 


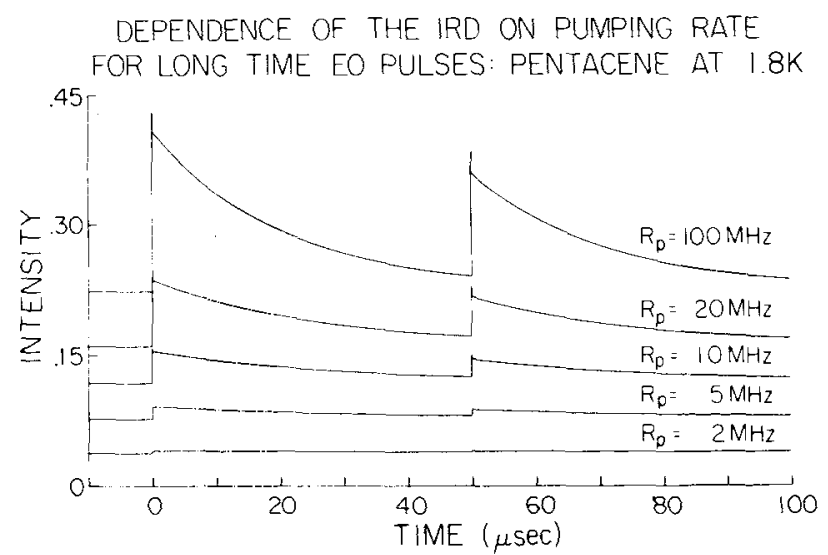

FIG. 11. The dependence of the IRD in pentacene at $1.8 \mathrm{~K}$ on the pumping rate for the EO method. Equations (43) and (46) are used to generate these computer plots considering both the on-resonance and off-resonance molecules [i.e., intensity here refers to $\left.\rho_{\rho p}^{\alpha}(t)+\rho_{p \phi}^{\beta}(t)\right]$. The parameters chosen are best fit values from experimental data (see Fig. 18). A transient overshoot (different from the overshoot, $a$, referred to in Fig. 8) occurs at the leading and trailing edges of the EO pulse. As described in the text, it results from the combination of incoherent emission and coherent pumping of different groups of molecules. The amplitude of the IRD at the end of the EO pulse $(t=50 \mu \mathrm{s})$ is smaller than the initial jump. This occurs because the ground state population of the off-resonance molecules has not recovered completely (i.e., population is still tied up in $\{|l\rangle\})$.

equations as before (41), (42) with $R_{p}$ set equal to zero. One obtains

$$
\begin{aligned}
\rho_{00}(t)= & \rho_{p p}\left(t_{e q}\right) \frac{\left(\Gamma_{p 0}-\Gamma_{l 0}\right)}{\left(T_{1 p}^{-1}-\Gamma_{10}\right)}\left\{e^{-\Gamma_{10} t}-e^{-t / T_{1 p}}\right\} \\
& -\left[1-\rho_{00}\left(t_{e q}\right)\right] e^{-\Gamma_{10} t}+1 .
\end{aligned}
$$

To complete the solution for the off-resonance time dependence of $\rho_{00}(t)$ we need to know two initial (boundary) values. These are (1) the equilibrium value of $\rho_{p p}$ on resonance, $\rho_{p p}\left(t_{e q}\right)$, and (2) the equilibrium value of $\rho_{00}$ on-resonance $\left[\rho_{00}\left(t_{0 Q}\right)\right]$. The first can be obtained from Eq. (43) at long times (compared to $\tau_{0}$ ). The second can be obtained from a solution of the differential Eq. (42). The solution $\left(R_{p} \neq 0\right)$ is:

$$
\begin{aligned}
\rho_{00}(t) & =\frac{R_{p}+T_{10}^{-1}}{\lambda_{+}-\lambda_{-}}\left[\left\{\frac{\Gamma_{10}+\lambda_{+}}{\lambda_{+}}\right\} e^{\lambda_{+} t}-\left\{\frac{\Gamma_{10}+\lambda_{-}}{\lambda_{-}}\right\} e^{\lambda_{-} t}\right] \\
& +\frac{1}{\lambda_{+}-\lambda_{-}}\left[\left(\lambda_{+}+\Gamma_{10}\right) e^{\lambda_{+} t}-\left(\lambda_{-}+\Gamma_{10}\right) e^{\lambda_{-} t}\right] \\
& +\frac{R_{p}+T_{10}^{-1}}{\lambda_{+} \lambda_{-}} \Gamma_{l 0} .
\end{aligned}
$$

Now $\rho_{00}\left(t_{\text {eq }}\right)$ can be found from Eq. (48) at long times (compared to $\tau_{0}$ ). Armed with the results of Eqs. (47) and (48) one can calculate the population of the ground state for any pulse width defined by $t^{\prime}$. Then one can use Eq. (43) to calculate the contribution of group $\alpha$ to the total signal as the laser frequency is switched back to the original group. The physical situation can be understood most easily by examining Fig. 12 where the total population $\rho_{p p}^{\alpha}(t)+\rho_{p p}^{\beta}(t)$ is plotted for various frequency switching pulse widths.
For pulse widths long compared to $\tau_{0}$ the two decay patterns are completely identical as also shown in Fig. 11. As the pulse width decreases, however, one notices that the height of the jump at the falling edge of the pulse is somewhat lower than the initial jump. Further reductions in pulse width make this difference more noticeable until finally the pulse width is short enough so that the increase in emission due to $\alpha$ being switched back into resonance is less than the decrease in emission due to $\beta$ being switched off-resonance. For these very short pulses where the contribution of $\alpha$ is negligible one can actually measure $1 / T_{1 p}$ from the decay of population at the falling edge of the pulse. These solutions of the coupled density-matrix equations for the EO and LADS experiments will be shown to fit all our available data on pentacene.

\section{RESULTS AND DISCUSSION}

\section{A. Narrow-band excitation: Site selection and vibrational relaxation}

At low temperature $(1.8 \mathrm{~K})$ mixed crystals of pentacene in $p$-terphenyl exhibit site splittings; four distinct lattice sites have been observed (see Fig. 13) in our crystals. Each site shows a vibronic progression of "sharp" lines $\left(1-3 \mathrm{~cm}^{-1}\right)$ both in absorption and emission (Fig. 14). In our work we have used several crystals of varying concentration $\left(10^{-5}-10^{-7} \mathrm{~m} / \mathrm{m}\right)$ and all of them show sites at energies $\left(\mathrm{cm}^{-1}\right): 16887\left(0_{1}\right), 16891\left(0_{2}\right)$, $17009\left(0_{3}\right)$, and $17069\left(0_{4}\right)$. These energies which are not vacuum corrected agree with the previous work of Marchetti et $a l .{ }^{44}$ and Bridge ${ }^{45}$ and are different by approximately $+4 \mathrm{~cm}^{-1}$ with the results of Wiersma et al.,$^{46}$ if the spectrum reported in their Fig. 1 is the real spectrum (i.e., without vacuum correction). If,

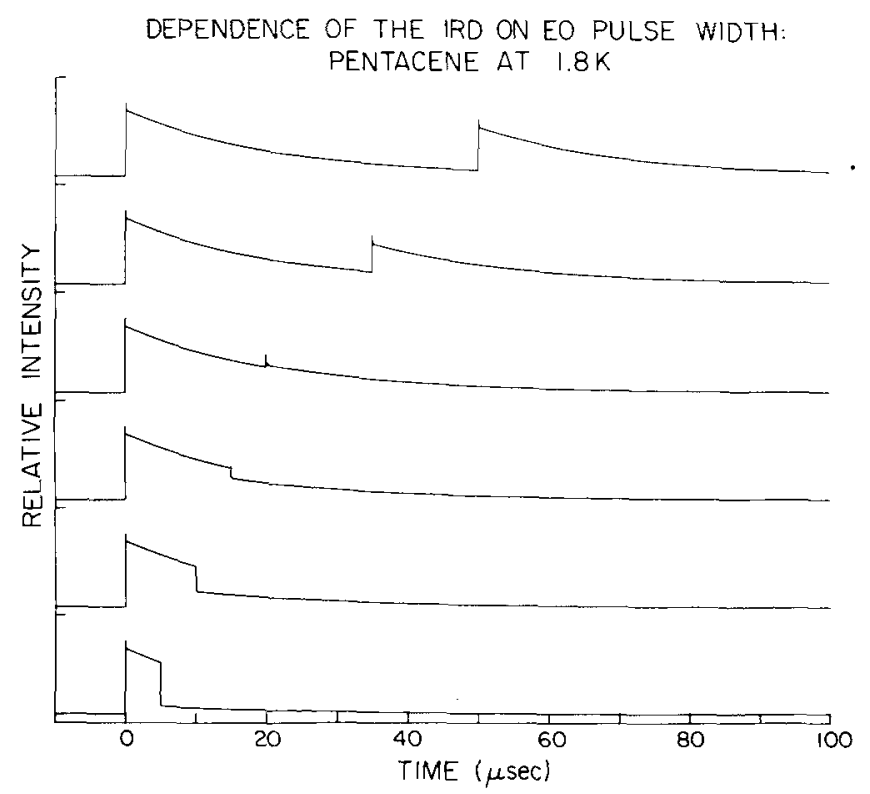

FIG. 12. Shown here is the dependence of the IRD on EO pulse width with $R_{p}=100 \mathrm{MHz}$ and all other parameters as in Fig. 11. The lifetimes of the slow decay seen after the leading and falling edges of the pulse are identical, regardless of pulse width. The fast decay seen for short pulses has a lifetime close to that obtained from LADS experiments. 


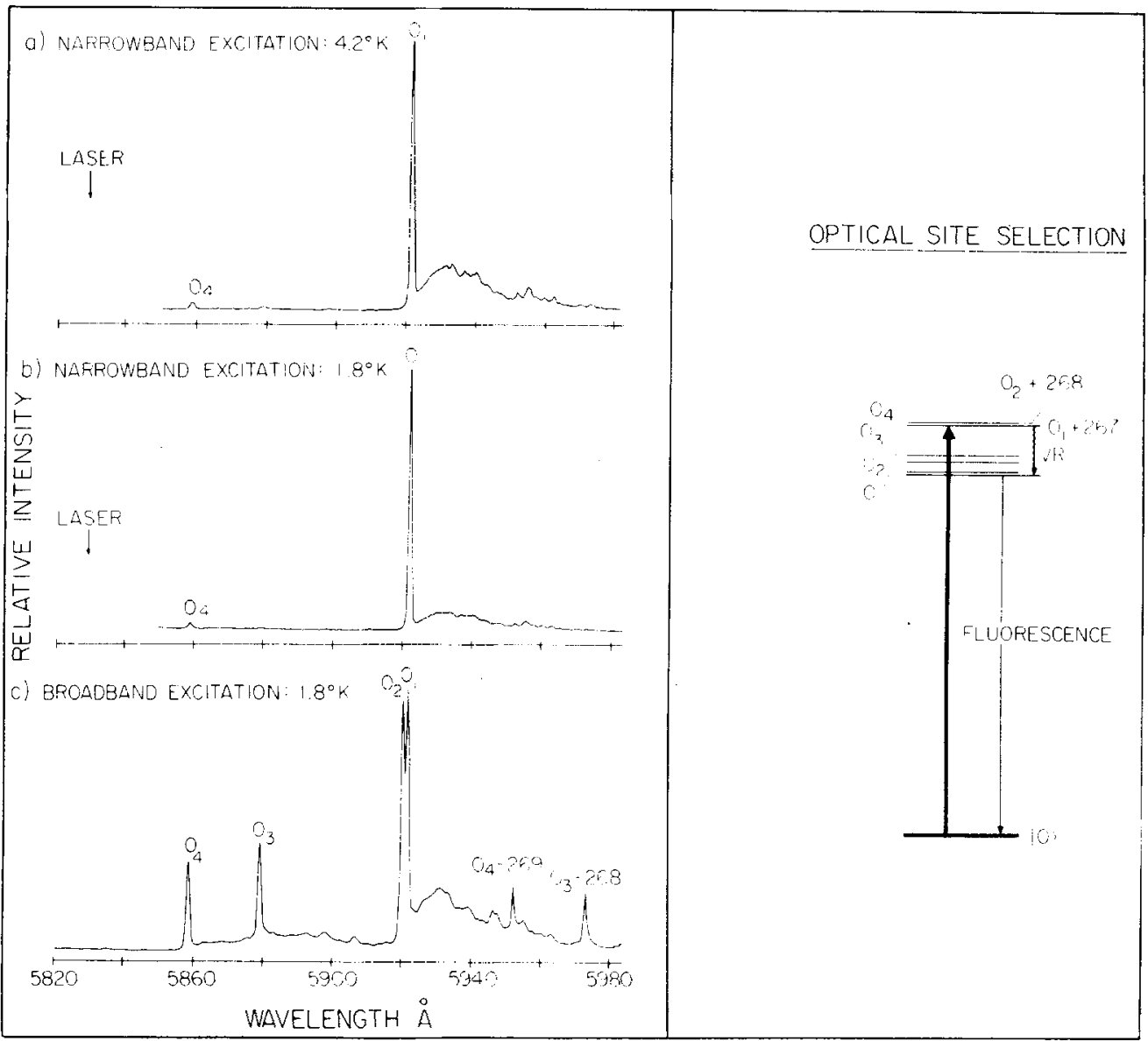

FIG. 13. Optical site selection in a mixed crystal of pentacene in $p$-terphenyl $(5$ $\times 10^{-6} \mathrm{~m} / \mathrm{m}$ ). Shown are (a) the emission spectrum of pentacene at $4.2 \mathrm{~K}$ with the single-mode laser on-resonance with a vibronic line of site $0_{1}, 267 \mathrm{~cm}^{-1}$ above the origin (see arrow). A very small amount (relative intensity $=0.03$ ) of emission is seen from $0_{4}$, otherwise narrowband excitation has selected $0_{1}$ molecules exclusively; (b) The emission spectrum of pentacene under identical conditions as in (a) except $\boldsymbol{T}=\mathbf{1 . 8}$ $K$. The phonon sideband at the $0_{1}$ origin is weaker but no other change in the spectrum occurs; (c) The spectrum of pentacene obtained with broadband excitation (Xenon lamp; $2000 \rightarrow 4000 \AA$ ) at $1.8 \mathrm{~K}$. Emission is seen from each site in this case. The spectrometer resolution was $0.7 \mathrm{~cm}^{-1}$ for all spectra. Shown on the right of the figure is an energy level scheme that is consistent with the above results.

however, their numbers are the corrected values, then all results agree to $\pm 1 \mathrm{~cm}^{-1}$.

A careful study of the spectra was done in order to identify these sites so that it is possible to make a meaningf il comparison of our time resolved spectra with all other available work on pentacene in $p$-terphenyl. Furthermore, these sites must be identified in order to establish whether or not intersite communication exists at these concentrations and temperatures since this process would introduce another dephasing channel, a primary concern of this paper. The optical properties of pentacene obtained in this work as well as a collection of pertinent other spectroscopic data in the gas and liquid phases are summarized in Table II.

To determine whether intersite communication occurs

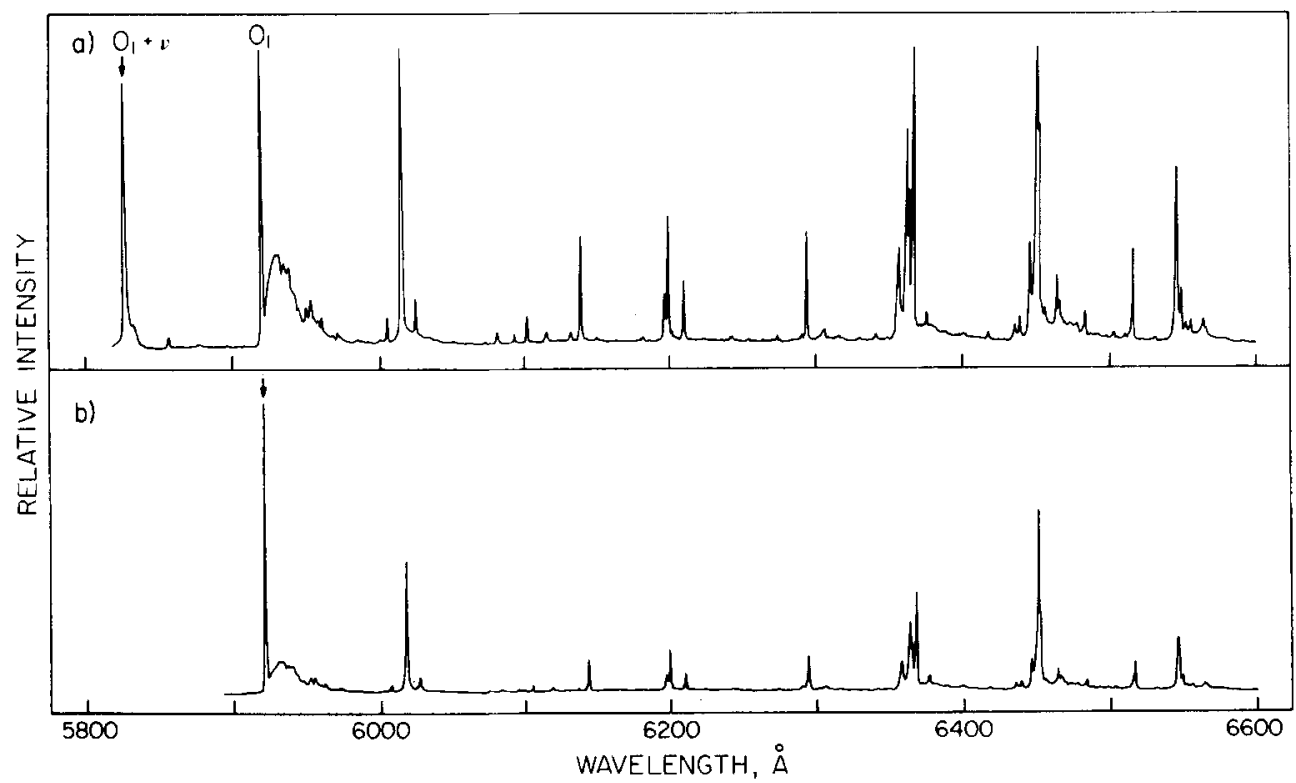

FIG. 14. (a) The emission spectrum of pentacene in $p$ te rphenyl with single-mode excitation (arrow) at $0, v(267$ $\mathrm{cm}^{-1}$ ) of site $0_{1}$; (b) The emission spectrum with the laser at 0,0 of $0_{1}$. Both spectra were obtained at $1.8 \mathrm{~K}$ and the linewidths shown here are determined by the spectrometer slitwidth $\left(1.4 \mathrm{~cm}^{-1}\right)$ 
TABLE III. Zeeman effect on IRD: Pentacene at $1.8 \mathrm{~K}$.

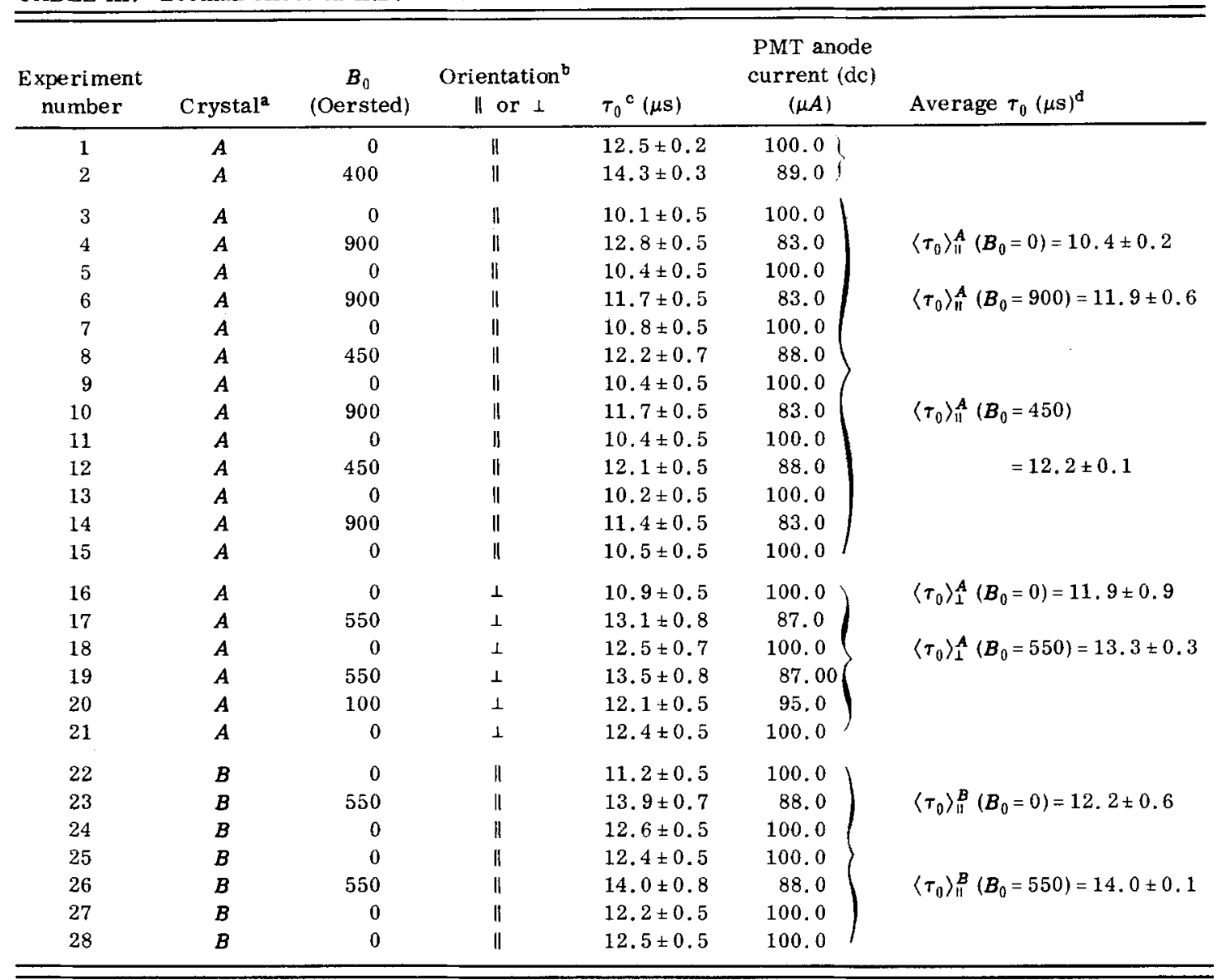

Crystal $A \approx 1 \times 10^{-5} \mathrm{~m} / \mathrm{m} ;$ crystal $B \approx 1 \times 10^{-6} \mathrm{~m} / \mathrm{m}$.

b $\|$ and $\perp$ refer to two distinct $\left(90^{\circ}\right.$ to each other) directions of the applied magnetic field, $B_{0}$, in the cleavage plane: $\|$ direction is close to the $b$ axis as identified by the growth habit of the crystal.

'The errors quoted in this column are standard deviations.

'The errors quoted in this column are standard deviations for the average values of $\tau_{0}$. Only data obtained on the same day at fixed laser frequency and under identical conditions are averaged.

in pentacene isolated in $p$-terphenyl several experiments were performed at guest concentrations of approximately $5 \times 10^{-6}$ and $5 \times 10^{-7} \mathrm{~m} / \mathrm{m}$. Following narrow-band excitation of the origin of site $0_{1}$ we obtained the emission spectrum at $1.8 \mathrm{~K}$ shown in Fig. 13 for the high concentration sample. An identical spectrum was obtained when we excited (narrow band) at the first vibronic line of $0_{1}(5829.8 \AA), 267 \mathrm{~cm}^{-1}$ above the origin. No emission was observed from sites $\mathrm{O}_{2}$ and $\mathrm{O}_{3}$ and only very weak emission from $0_{4}$ (relative intensity $=0.03$ ) although each origin lies below the vibronic line in energy (see Table II). The spectra for the low concentration sample at $1.8 \mathrm{~K}$ and both samples at $4.2 \mathrm{~K}$ matched the spectrum mentioned above except that the phonon sideband of the $0_{1}$ origin was more intense and showed structure at higher temperature. The weak emission at $0_{4}$ also appeared at very low laser power indicating that power broadening of the $0_{1}$ vibronic line can not be responsible for the communication observed with $0_{4}$. It is interesting to note that site 4 is the closest in energy to the excitation energy and its appearance may therefore indicate the importance of near resonance energy transfer to $0_{4}$. Although we cannot rule out that the site is due to an impurity, the fact that it has the same vi- bronic progression ${ }^{45}$ leads one to believe that it is due to pentacene.

Also, the emission spectrum for the high concentration sample at $1.8 \mathrm{~K}$ following broadband excitation (xenon lamp, UV bandpass) was obtained. In this spectrum (see Fig. 13) emission is seen from each site origin and vibronic lines can be identified. Several conclusions can be drawn from these experiments.

First, vibrational relaxation within the lowest excited singlet manifold is fast enough so that emission is observed from the origin only. Second, at these concentrations $\left(\leq 5 \times 10^{-6} \mathrm{~m} / \mathrm{m}\right)$ and temperatures $(\leq 4.2 \mathrm{~K})$ spectral diffusion processes such as excitation exchange among sites are not competing effectively with vibrational relaxation because emission is seen from the site $0_{1}$ origin predominantly (with narrow-band excitation) although we are exciting above the origins of all the other sites. Although the vibronic line for site $\mathrm{O}_{2}$ found at $5827.9 \AA$ is only $5.6 \mathrm{~cm}^{-1}$ above the corresponding vibronic line for $\mathrm{O}_{1}$ (excited by the laser), no communication between these two sites occurs. Thus, with narrow-band excitation individual sites can be selected and their interaction with the environment can be studied 


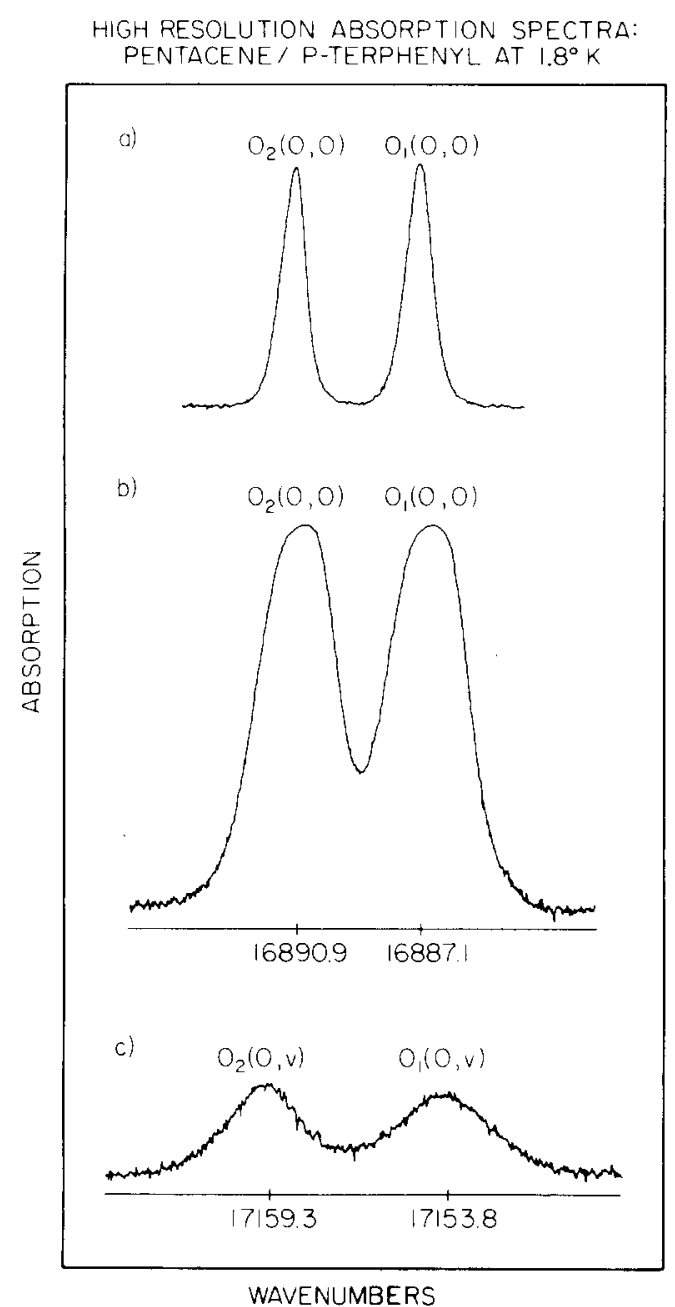

FIG. 15. High resolution absorption spectra for pentacene in $p$-terphenyl at $1.8 \mathrm{~K}$. Shown are (a) $0_{1}$ and $0_{2}$ origins at low concentration $\left(\approx 1 \times 10^{-6} \mathrm{~m} / \mathrm{m}, 1 \mathrm{~mm}\right.$ crystal); (b) $0_{1}$ and $0_{2}$ origins at high concentration $\left(\approx 5 \times 10^{-6} \mathrm{~m} / \mathrm{m}, 3 \mathrm{~mm}\right.$ crystal $)$; (c) $0_{1}$ and $0_{2}$ vibronic lines at high concentration.

free from complications due to intersite effects. Even though intercommunication between sites may not influence the spectra, it is possible that the $T_{2}^{\prime}$ processes involving elastic site communications will be influenced and thus alter the decay rate of coherent transients. Therefore one must be careful about correlating the emission spectra with $T_{2}$-type processes that involve other sites. Finally, there appears to be structure superimposed on the phonon sideband continuum near the origin of $0_{1}$ which may indicate the presence of pseudolocalized or resonance phonon modes in the lattice. ${ }^{47,48}$ These are not seen in our absorption spectrum or in the spectrum of Aartsma et al. ${ }^{49}$ However, there is evidence that phonon structure can be markedly different in absorption and emission in very similar mixed crystals. ${ }^{50}$ These modes can be very important dephasing channels and we will address this point in more detail in a later section.

Because vibrational relaxation is fast enough so that emission following excitation into the vibronic line is seen only from the site origin, we decided to carefully measure the absorption linewidths of the $0_{1}$ origin and its vibronic line $267 \mathrm{~cm}^{-1}$ to higher energy. Initial results with spectrometer resolution of $0.8 \mathrm{~cm}^{-1}$ revealed that the origin can be fit to a Gaussian whose linewidth increases with sample concentration and temperature. The vibronic line, on the other hand, appeared Lorentzian. Because the absorption at the origin was high $(\approx 90 \%)$, self-absorption (which makes the observed lifetime longer than the actual lifetime) and scattering ef fects could introduce artifacts in the measurement.

With very low concentration crystals $\left(O D \sim 10^{-2}\right)$ we repeated these linewidth measurements using a high resolution spectrometer (Spex double monochromator, $0.75 \mathrm{~m}, 0.09 \mathrm{~cm}^{-1}$ resolution). The results are depicted in Fig. 15 for the 0,0 and $0, v$ transitions of both $0_{1}$ and $0_{2}$. The origins show dramatic broadening as the concentration increases. For the lowest concentration crystal, the linewidths (FWHM) of the $0_{1}$ and $0_{2}$ origins are $0.7 \pm 0.1 \mathrm{~cm}^{-1}$. At the highest concentration used, these widths increase by more than a factor of 2. Consistently, we found that the vibronic lines of $O_{1}$ and $O_{2}$ are broader than the 0,0 transition. From the coherent transient experiments we know that the broadening of the $0_{1}$ origin is inhomogeneous in nature. If the vibronic line is homogeneously broadened, as it appears to be, then we can obtain $T_{2}$ directly. We were therefore encouraged to computer fit the data carefully in order to establish whether the lines are Lorentzian or Gaussian. The best fit for the $\mathrm{O}_{2}$ vibronic line gives a Lorentzian with a width (FWHM) of $1.99 \pm 0.03 \mathrm{~cm}^{-1}$. If this width is entirely due to vibrational relaxation, then $T_{1 v}=2.7 \mathrm{ps}$, much different from previous results. ${ }^{44}$ We shall return later to this point in Sec. VI.

\section{B. Optical dephasing: nutation and OFID}

As discussed earlier, electronic dephasing times $\left(T_{2}\right)$ can be obtained from optical nutation and OFID experiments under the proper conditions. Dilute $\left(10^{-6} \mathrm{~m} / \mathrm{m}\right)$ crystals of pentacene in $p$-terphenyl of good optical quality were used to observe the optical nutation (Fig. 16) and OFID (Fig. 17) at $1.8 \mathrm{~K}$ utilizing the EO method of frequency switching.

The data in Fig. 16 were obtained with high (singlemode) laser power ( $20 \mathrm{~mW}$ ) exciting the electronic origin $0_{1}$ at $16887 \mathrm{~cm}^{-1}$. The EO switching frequency measured using a scanning confocal etalon was $26 \mathrm{MHz}$ (see Fig. 2). Theoretical curves for the data points were generated (solid lines) using Eq. (19) which considers averaging the result for $r_{2}(t)$ over only the inhomogeneous linewidth of the transition (top), and using Eq. (29) which considers both inhomogeneous linewidth averaging as well as averaging over the laser field spatial profile (bottom of Fig. 16). It is apparent how important averaging over the laser beam, which essentially causes the transient to damp after the first oscillation, is for fitting the signal adequately. For both plots, the same values of the Rabi frequency $(\chi=\mu \cdot \epsilon / \hbar)$ and $T_{2}$ were used and the best fit values for the bottom plot are $230 \pm 10 \mathrm{MHz}$ (which corresponds to a nutation time of $27.3 \mathrm{~ns}$ ) and $45 \pm 2 \mathrm{~ns}$, respectively. Thus, our experimental results verify the assumption of high power $(\chi$ $\left.\gg 1 / T_{2}, 1 / T_{1}\right)$ which was made to simplify the form of the density matrix solution for $r_{2}$ and show that with the ap- 
OPTICAL NUTATION: PENTACENE AT $1.8 \mathrm{~K}$

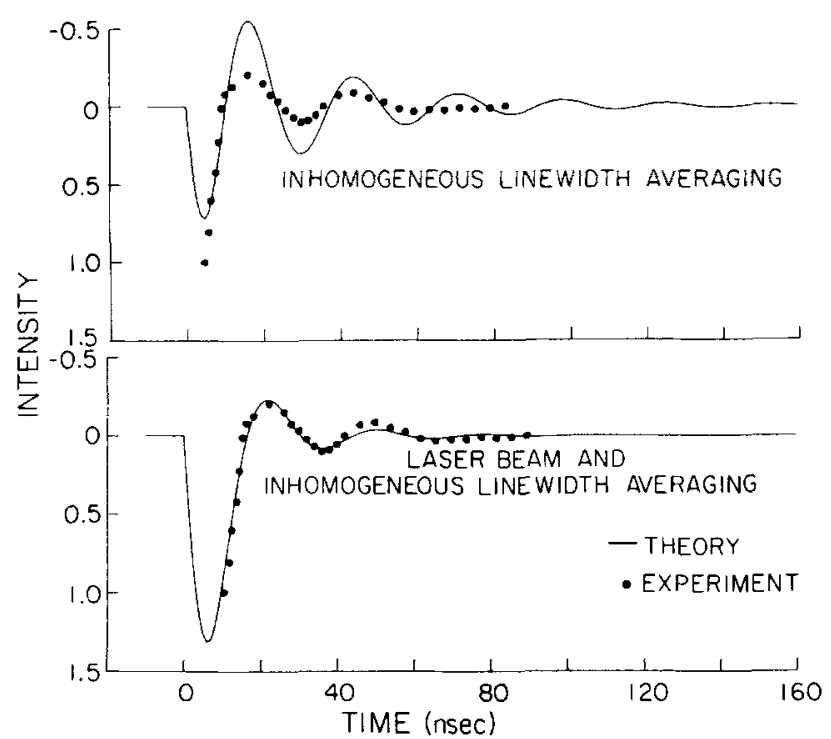

FIG. 16. Shown here are experimental data points and theoretical curves for the optical nutation in pentacene at $1.8 \mathrm{~K}$. In each plot, the vertical axis corresponds to relative absorption (arbitrary units) and $t=0$ is taken to be the switching time of the EO pulse. The top plot considers only inhomogeneous line shape averaging [Eq. (19) multiplied by the error function shown in Eq. (28)]. The bottom plot considers averaging over both the inhomogeneous line shape and the laser beam spatial profile (Eq. 29). The fit is quite good in the latter case and provides values of $27.3 \pm 1.3 \mathrm{~ns}$ for the nutation time and $45 \pm 2 \mathrm{~ns}$ for $T_{2}$.

propriate averaging over the laser beam, optical nutation measurements can provide $T_{2}$. This $T_{2}$, as we shall see later, can also be obtained from OFID and echo experiments. Since the spatial profile of the laser has been characterized (Fig. 3) the laser intensity can be determined, and thus the laser field strength. As emphasized by Shoemaker and Van Stryland ${ }^{23}$ careful nutation measurements provide the Rabi frequency which allows a direct determination of the transition dipole moment. Knowing the power of the laser $P_{L}$ in milliwatts, the Rabi frequency $\chi$ in $\mathrm{MHz}\left(10^{6} \mathrm{c} / \mathrm{s}\right)$ and $B_{L}$ in microns from the laser spatial profile, one obtains the following expression for $\mu$ in Debye:

$$
\mu(\mathrm{D})=4.06 \times 10^{-4} \frac{\chi B_{L}}{\left(\frac{\eta_{b}^{2}+2}{3}\right) \sqrt{P_{L}}} .
$$

The Rabi frequency $\chi$ (in linear units) obtained from Fig. 16 is $36.6 \pm 1.6 \mathrm{MHz}$. The terphenyl $b$-axis refractive index $\eta_{b}=1.687$. We have used the Lorentz local field approximation to obtain the laser field inside the crystal. For a single mode power of $17 \pm 1 \mathrm{~mW}$ at the crystal (after correcting for reflection losses from the optical elements; only $3 \mathrm{~mW}$, i. e., total of $15 \%$ ), and using Eq. (49) we obtain for pentacene in $p$-ter.phenyl at $1.8 \mathrm{~K}$, a value for $\mu$ of $0.7 \pm 0.1 \mathrm{D}$. This value is less than our earlier estimate of $1.5 \mathrm{D},{ }^{18}$ where no field correction was made. The $T_{2}$ value obtained from the same transient (Fig. 16) agrees with our OFID measurements ${ }^{51}$ (described later in detail) and with the results obtained independently by Wiersma et al. ${ }^{52}$ Their value of $\mu$ obtained from the OFID is $1.5 \mathrm{D}$, but in a recent work [Ref. $52(\mathrm{c})]$, they obtained $1 \mathrm{D}$.

We have studied the nutation signal at various EO switching frequencies (the amount by which the laser frequency is changed, not the rate) and have observed an increase in the initial amplitude of the transient as the frequency is increased. No change in the Rabi fre quency $(\mu \cdot \epsilon / \hbar)$ could be detected. This is very impor tant since it confirms the absence of complications due to OFW transients from off resonance molecules which at this power decay extremely rapidly (see Fig. 7). This increase in nutation amplitude can be explained by realizing that at low switching frequency the laser is not "moved" completely off-resonance with the original power-broadened group, allowing it to weakly pump the original molecules. At very low switching frequencies (i. e., within the homogeneous linewidth; 7.1 MHz) no transient is observed, as expected. In addition to this frequency shifting effect on the nutation amplitude, the signal is very sensitive to changes in temperature. This effect will be discussed in a following section where we consider phonon processes as effective dephasing channels.

The data shown in Fig. 17 for the OFID were obtained at very low laser powers $(400 \mu \mathrm{W})$ exciting the same electronic origin in the same crystal under identical conditions of temperature and laser frequency as in Fig. 16. Unlike the nutation, the computer fit (solid line) using Eqs. (36) and (38) is very good when we only consider averaging over the inhomogeneous linewidth. Since the OFID transient originates from molecules offresonance its decay may not be influenced by the laser. To be specific, averaging over the laser profile gives

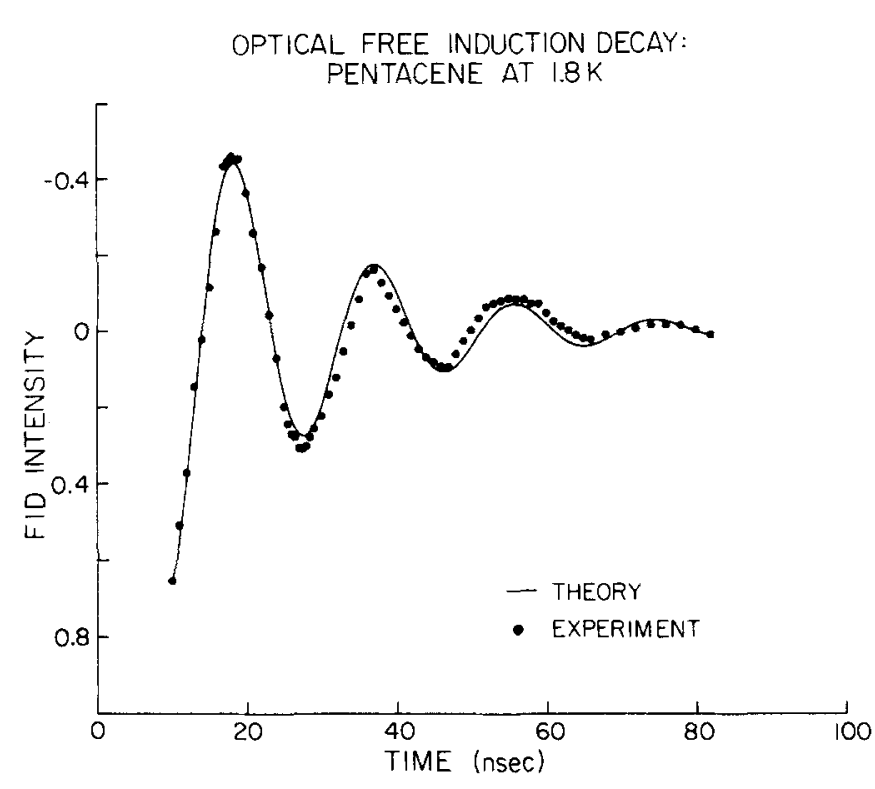

FIG. 17. The optical free induction decay observed in pentacene $\left(0_{1}\right.$ origin) at $1.8 \mathrm{~K}$ and $400 \mu \mathrm{W}$ of laser power. Here the experimental data points and theoretical curve (Eq. 38) are in excellent agreement. The power broadening term in Eq. (35) was calculated by scaling the Rabi frequency from the nutation by the square root of the ratio of the laser power used in each experiment. (The nutation and the OFID were taken under identical conditions on the same crystal.) The computer fit then provided a value of $45 \pm 2 \mathrm{~ns}$ for $T_{2}$ from the observed transient decay. 
an effective Rabi frequency which affects the decay of transients occurring on-resonance. To obtain the fit in Fig. 17 we fixed $T_{1 p}$ at $25 \pm 1$ ns (see Table II and Sec. $\mathrm{VC}$ ) and scaled the Rabi frequency in the power broadening term for the OFID from that obtained in the nutation experiment (done under identical conditions) by the ratio of the square root of the observed laser intensities in each experiment. The fit then provided a value of $45.3 \pm 2 \mathrm{~ns}$ for $T_{2}$ consistent with our nutation experiment. Thus, our results show that at $1.8 \mathrm{~K}$, the pure dephasing time $T_{2}^{\prime}$ is essentially infinite for pentacene in the terphenyl host since $T_{2}=2 T_{1 p}$ [see Eq. (11)]. We shall see later that this is no longer true at higher temperature.

In Fig. 17, the heterodyne beat frequency is $53 \mathrm{MHz}$. It depends linearly on the EO switching voltage (here $100 \mathrm{~V}$ ) and agrees with the experimentally measured dispersion of the $\mathrm{AD}^{*} \mathrm{P}$ crystal $(0.56 \mathrm{MHz} / \mathrm{V})$ determined using the etalon setup mentioned earlier (Fig. 2). We should mention that a considerable improvement in OFID signal quality is observed when linear polarizers were placed immediately before and after the Dewar windows. This is reasonable since slight depolarization can occur from irregularities in the optical surfaces used to direct the laser beam to the sample, and from strain-induced birefringence in the Dewar windows themselves.

In these frequency switching experiments, where both off-resonance and on-resonance molecules are observed simultaneously, the dependence of the observed transients on laser power is very dramatic. At low power $(<1 \mathrm{~mW})$ the OFID signal from off-resonance molecules dominates and the optical nutation from molecules switched into resonance provides a weak background ab sorption whose Rabi frequency $<1 / T_{2}$. On the other hand, at high power $(\geq 20 \mathrm{~mW})$ the optical nutation from on-resonance molecules dominates since the power broadening term in the OFID expression [Eq. (36)] causes the observed damping time for the OFID of off-resonance molecules to be much shorter than $T_{2}$ (see Fig. 7). In other words, power broadening allows a larger fraction of the total frequency distribution within the inhomogeneous linewidth to "participate" in the coherent pumping process and this distribution will cause a faster decay of the transient due to inhomogeneous dephasing.

In the OFID experiments at $400 \mu \mathrm{W}$ the power broadening term contributes only $10 \%$ to the observed decay. However, at $20 \mathrm{~mW}$, the observed decay would be approximately $7 \mathrm{~ns}$ and within the fall time of the switching pulse. Thus, an accurate measurement of $T_{2}$ from the OFID requires very low laser power while for the nutation experiment an accurate value can be obtained at high power if averaging over the laser beam spatial profile is done.

The homogeneous linewidth $\left(1 / \pi T_{2}\right)$ obtained from the nutation and OFID is $7.1 \pm 0.4 \mathrm{MHz}$. This value is over one thousand times smaller than the inhomogeneous linewidth (see Sec. VA). The fact that this homogeneous broadening is very small has turned out to be a valuable result since we can use EO and LADS techniques to study the effects of environment (see Sec. V F) and excess vibrational energy upon radiationless relaxation processes which are important for large molecules like pentacene. If the homogeneous linewidth was a factor of 10 larger we would be forced to do these experiments on the picosecond time scale.

The important points about this homogeneous broadening are (1) it is entirely determined by the relaxation rate of the primary level $|p\rangle$ and that there is no unusual intramolecular dephasing process at low temperature $\left(1.8 \mathrm{~K}\right.$ ) and $(2)$ knowing $T_{1 p}$ of sites $0_{3}$ and $0_{4}$ (Table II) and assuming that $T_{2}^{\prime-1}$ is zero (mimicking the $0_{1}$ site) the width must be $16.4 \mathrm{MHz}$, and that is why these sites have relatively weaker transients that decay faster than $0_{1}$.

\section{Radiative and radiationless relaxation: IRD}

\section{Broadband excitation}

In the molecular eigenstate picture, broadband excitation prepares a large number of $|m\rangle$ states simultaneously. As mentioned before, the observed decay should have a "fast" component and a slow component whose decay time is essentially characteristic of $\psi_{m}$ of Eq. (1). If many states are involved in the coupling then the amplitude of the slow component will be insignificant compared to the initial fast decay and the total decay could appear as a single exponential (statistical limit). On the other hand, for a sparse level structure narrow-band excitation should select $|m\rangle$ states and their decay will reflect the contribution of the primary level to the molecular eigenstate [Eq. (1)].

Broadband excitation of pentacene in $p$-terphenyl was performed in three different ways: using a nitrogen laser pumped pulsed dye laser; a nitrogen flash lamp, and a multimode $\mathrm{cw}$ dye laser. Using the nitrogen laser pumped dye laser ( $5 \mathrm{~ns}$ pulse) with $18 \mathrm{GHz}$ bandwidth a lifetime $\left(T_{1 p}\right)$ of $23 \pm 3$ ns was measured for pentacene in $p$-terphenyl exciting the electronic origin $0_{1}$ at 16887 $\mathrm{cm}^{-1}$ at a temperature of $1.8 \mathrm{~K}$. This value agrees with previous measurements (see Table II) yet is a factor of three longer than that found from gas phase experiments (see Table II). Using a $\mathrm{N}_{2}$ flash lamp $(3650 \pm 50 \AA)$ careful lifetime measurements were performed by N. J. Bridge $^{45}$ at $4 \mathrm{~K}$ for each of the electronic origins of a crystal supplied by us. He found that the two highest energy sites $\left(0_{3}, 0_{4}\right)$ have lifetimes of $9.7 \pm 1$ ns while the lower energy sites $\left(0_{1}, 0_{2}\right)$ have lifetimes of $25.7 \pm 1 \mathrm{~ns}$. Within detection limits the decays appear as single exponentials. Also, similar vibrational progressions for each of the sites were observed in the spectra consistent with the spectrum depicted in Fig. 14. The third kind of broadband excitation which utilized a multimode (240 $\mathrm{GHz}) \mathrm{cw}$ dye laser and LADS gave the same $T_{1}$ decay time; its importance will be discussed later.

From these $T_{1}$ type experiments done with broadband excitation sources, two points can be made: (a) the lifetimes of origins $0_{1}$ and $0_{2}$ are constant from 1.8 to 4. $2 \mathrm{~K}$ (this will also be shown to be true for the narrowband excitation) and (b) because the observed decay is a single exponential over many lifetimes there is no apparent evidence of the long-lived tail that characterizes the decay of $\psi_{m}$ following broadband excitation. Point (a) will be important later (Sec. V F) when temperature dependent dephasing processes are discussed. 


\section{Narrow-band excitation: EO and LADS}

Our first experiments (I) on pentacene utilizing narrow-band excitation ( $\pm 3 \mathrm{MHz}$ ) and the EO frequency switching method showed a fast buildup in emission intensity followed by a slow decay $(\approx 15 \mu \mathrm{s})$ occurring at the leading and falling edges of the EO pulse. This decay could be fit to a single exponential over three lifetimes and the transient pattern is characterized by several important observations that span the short (transient coherence regime) and the long (steady-state coherence regime) time scales. The short time behavior of the pattern manifests the coherence effects discussed previously (Sec. V B) and will be examined later when we discuss the role of temperature. The decay pattern at long times (i.e., $t \gg T_{2}$ ) will now be discussed.

The decay at the falling edge of the pulse is very sens itive to pulse width which in this regime is much larger than $T_{2}$. Figure 18 shows IRD waveforms (data points) and computer fits (solid lines) utilizing Eqs. (43)-(48) from the density matrix solution for $\rho_{p p}(t)$ in the steadystate coherence regime. Here, the data was obtained with a signal averager time resolution of $200 \mathrm{~ns}$ and so the overshoot predicted in Figs. 11 and 12 is averaged out at the leading and falling edges of the EO pulse. This overshoot has been observed using a fast $(>1 \mathrm{GHz}$ bandwidth) photomultiplier and a sampling scope. As th pulse width decreases, the observed decay approaches that found in the broad band experiments. Although this effect has been explained in Sec. IV, up until this point we have neglected the fact that as the pulse width decreases, its Fourier transform (in frequency) broadens. Therefore, the effective bandwidth of the laser is wider than its cw single-mode value and thus it may span a different energy width in the molecular excitation process. In Appendix A we show Fourier transforms for narrow and wide laser pulses to illustrate the effect. In general, it is therefore not surprising that although one is exciting with a single-mode source, the broadband prepared state may be recovered as the pulse width decreases, especially if the homogeneous width of $|m\rangle$ 's is comparable to their separation.

From Fig. 18 one can see that the buildup in emission intensity at the falling edge of a long EO pulse does not equal the buildup at the leading edge if the decay has not reached a steady-state value. As explained earlier (Sec. IV), this effect is due to population not having returned completely to the ground state, 10$\rangle$.

To obtain the fits shown in Fig. 18, only one parameter, $T_{1 p}$, was fixed. All others were optimized by the nonlinear least squares regression analysis program described in Sec. III. We set $T_{1 p}$, the lifetime of the primary level equal to $25 \pm 1$ ns as obtained from our measurements. The computer fit provided values of $20.4 \pm 1.5 \mu \mathrm{s}$ for $\Gamma_{p l}^{-1}$ and $37.6 \pm 2.6 \mu \mathrm{s}$ for $\Gamma_{i 0}^{-1}$. The fit for the observed decay gave a value of $\tau_{0}=19.6 \pm 1.5 \mu \mathrm{s}$ and this decay was insensitive to the initial estimate of $R_{\rho}$, the pumping rate, if we chose a value large enough $(\geq 100 \mathrm{MHz}$ ) such that we were in the flat portion of the observed lifetime vs pumping rate curve (Fig. 10). This choice of $R_{p}$ is consistent with our observation of the decay time being insensitive to laser power. At very small pumping rates where Fig. 10 predicts a longer decay time the overshoot (referred to as " $a$ " in
Fig. 8) becomes very small and so the decay time becomes difficult to measure accurately. Over the laser power range that we could measure decay time accurately $(1-50 \mathrm{~mW})$, the decay time did not vary significantly. Considering the value of $\Gamma_{p l} / \Gamma_{10}$ in Fig. 10 the experimental results are consistent with the theoretical predictions.

The rate of population loss from the $\{|l\rangle\}$ manifold, $\Gamma_{30}$ corresponds to a lifetime of $37.6 \pm 2.6 \mu \mathrm{s}$, which is very close to the lifetime of the lowest triplet state in pentacene measured at low pressures in the gas phase (see Table I). So, it is reasonable to conclude that the $\{|l\rangle\}$ manifold does indeed involve triplet states. Of course, it is possible that hot singlets happen to have similar lifetimes.

The rate at which the $\{|l\rangle\}$ manifold receives population from the primary level $|p\rangle$ is $\Gamma_{p l}$. In the steady state coherence regime it represents an average of all the processes connecting $|p\rangle$ with $\{|l\rangle\}$. This averagt rate, however, does not mean that an indirect mechanism involving an initial fast crossing between $|p\rangle$ and vibrational levels of the $\{|l\rangle\}$ manifold isoenergetic with $|p\rangle$ followed by slow relaxation to lower levels in the manifold is indistinguishable from the simple description shown in Fig. 4. In fact, this mechanism would provide a completely different emission transient from the one observed as will be discussed later.

We observed no dependence of the decay of the IRD with EO switching frequency although the initial amplitude varied linearly in most experiments. As discussed earlier for the nutation, the initial amplitude should decrease if one does not switch completely outside of the power-broadened homogeneous linewidth. The observation of no change in decay rate with switching frequency up to the maximum value of $65 \mathrm{MHz}$ is interesting when one considers another observation. As the laser fre-

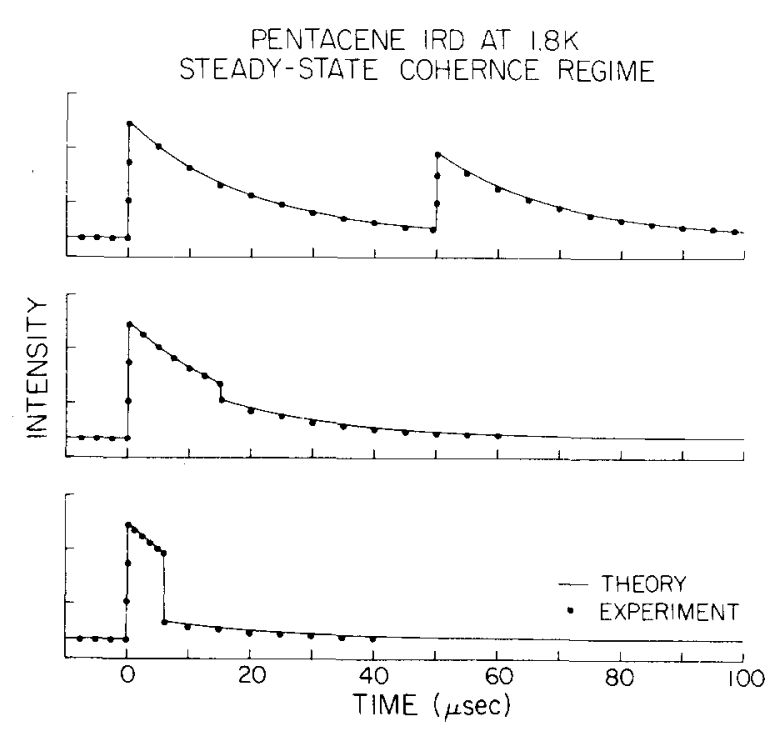

FIG. 18. Pentacene IRD experimental data points and theoretical fits as a function of $E O$ pulse width at $1.8 \mathrm{~K}$. The steadystate coherence regime density matrix equations for $\rho_{p p}(t)$, Eqs. (43) and (46), were used to determine the contribution of resonance and off-resonance molecules to the total emission signal. The computer fits provided values of $20.4 \pm 1.5 \mu$ s for $\Gamma_{p l}^{-1}$ and $37.6 \pm 2.6 \mu \mathrm{s}$ for $\Gamma_{l 0}^{1}$ with $\Gamma_{p 0}$ fixed at $40 \mathrm{MHz}$. 


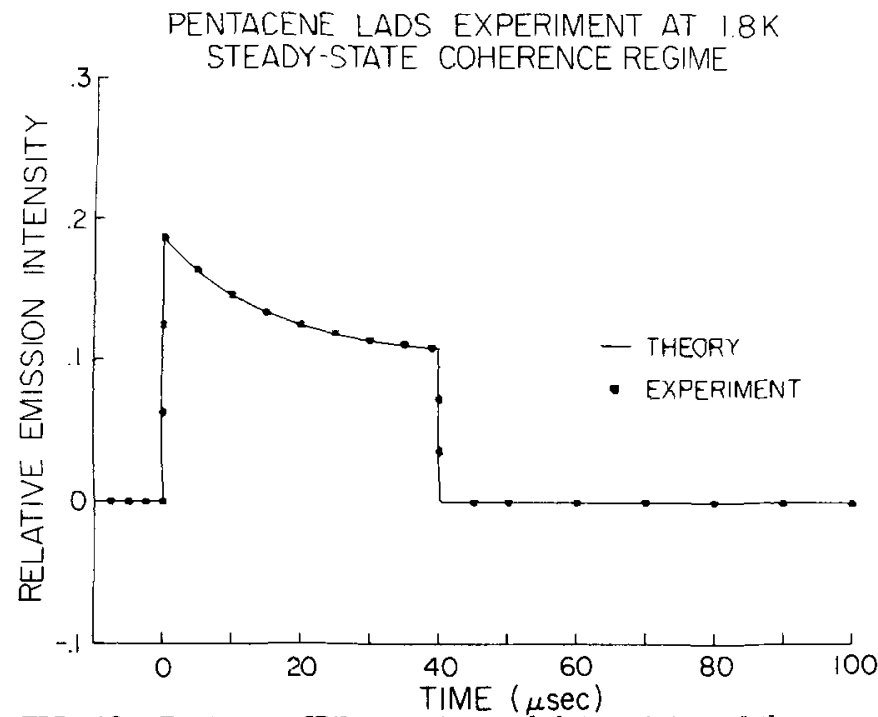

FIG. 19. Pentacene IRD experimental data points and the theoretical fit for LADS excitation pulses at $1.8 \mathrm{~K}$. Here $\rho_{\rho p}(t)$ is calculated using Eqs. (43) and (46) for only the one group of molecules excited by the narrow-band laser. The computer fit provided values of $16.3 \pm 1.2 \mu \mathrm{s}$ for $\Gamma_{p l}^{-1} ; 26.6$ $\pm 1.5 \mu \mathrm{s}$ for $\Gamma_{l 0}^{-1}$ and $14.7 \pm 1.0 \mu \mathrm{s}$ for the observed decay time, $\tau_{0}$. The crystal of pentacene in $p$-terphenyl used in this experiment was different from the one used for the results shown in Fig. 18 but was approximately the same concentration $\left(1 \times 10^{-6} \mathrm{~m} / \mathrm{m}\right)$.

quency is tuned within the inhomogeneous linewidth $(\approx \mathbf{5 0}$ $\mathrm{GHz}$ ) we measure decay rates that vary in the range $11-$ $20 \mu$ s depending on the frequency and the samples used. Thus, with these two observations there appears to be an anisotropy in the decay channel(s) connecting the pri- mary level $|p\rangle$ to the $\{|l\rangle\}$ manifold that occurs for energy separations outside the range of the frequency switched laser. The inhomogeneous linewidth of the 0 $\rightarrow p$ transition reflects a distribution of local environments for the isolated pentacene molecules, but it is not obvious whether the observation of decay rate differences implies an environmental effect on the intramolecular process connecting $|p\rangle$ with $\{|l\rangle\}$ or that the coupling to individual levels is nonuniform even in the absence of any environment (i.e., a completely isolated system). Molecular beam experiments are being planned to clear up this point.

The emission signal seen for pentacene at $1.8 \mathrm{~K}$ using LADS excitation (Figs. 19 and 20) differs dramatically from the IRD seen for the EO method (Figs. 18 and 20). In Fig. 19, data points were fit using the density matrix solution for $\rho_{p p}(t)$ in the steady-state coherence regime considering only one ensemble of molecules since the laser is shut off following the slow decay and not switched to a new group of molecules as in the EO experiment. The slow decay is somewhat faster here $(14.7 \pm 1.0 \mu \mathrm{s})$ but within the range of the EO experiments. When EO and LADS experiments were performed under identical conditions of laser frequency and sample concentration, identical decays were measured (see Fig. 20).

The fast decay on the lalling edge of the LADS pulse has a lifetime of $24.5 \pm 2 \mathrm{~ns}$ which is in excellent agreement with $T_{1 p}$ obtained from broadband experiments. This decay at the falling edge is a single exponential after deconvolution knowing the exact excitation pulse shape. Furthermore, there is no change in the long and short decays lobtained from these LADS experi-

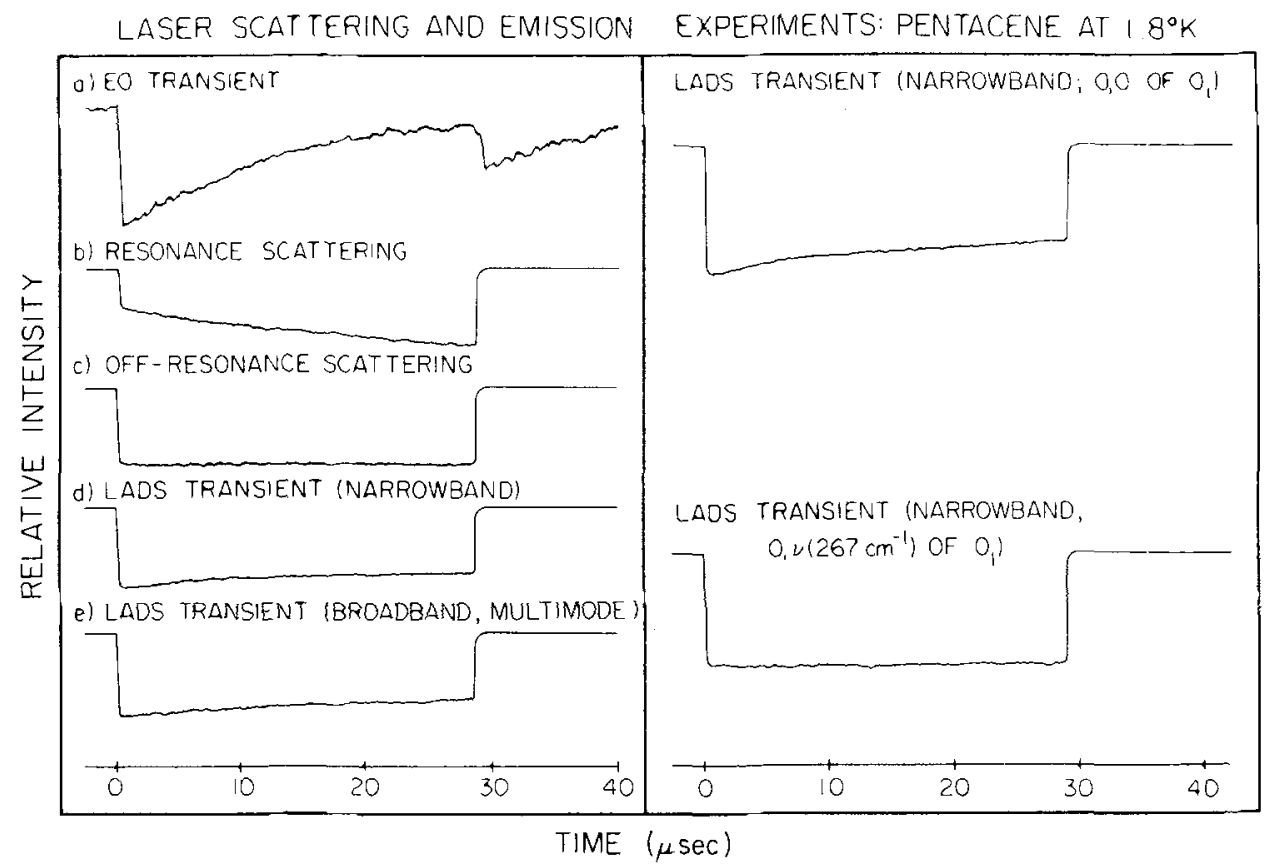

FIG. 20. Emission and laser scattering experiments for pentacene at $1.8 \mathrm{~K}$. Shown are (2) the emission transient observed with the EO technique; $\tau_{0}=13.9 \pm 0.4 \mu \mathrm{s}$; (b) the laser scattering signal (on-resonance) observed with narrow spectrometer slits $(0.7$ $\mathrm{cm}^{-1}$ ). Here, the slow buildup time is $13.7 \pm 0.3 \mu \mathrm{s}$; (c) the laser scattering signal off-resonance $\left(-3.5 \mathrm{~cm}^{-1}\right)^{84}$; (d) the enission transient observed with the LADS technique (single mode): $\tau_{0}=13.8 \pm 0.4 \mu \mathrm{s}_{i}$ (e) the emission transient observed with multimode (broad band) LADS excitation; $\tau_{0}=15.6 \pm 0.6 \mu \mathrm{s}$. At the top right of the figure is shown the single-mode LADS emission transient exciting at a differeat frequency from experiment (d) but still within the inhomogeneous linewidth of the $a_{\mathrm{f}}$ site origin; here $\tau_{0}$ $=14.6 \pm 0.5 \mu \mathrm{s}$. At the bottom right of the figure is shown the LADS emission signal exciting at the $0_{1}$ vibronic line $267 \mathrm{~cm}^{-1}$ above the origin. 


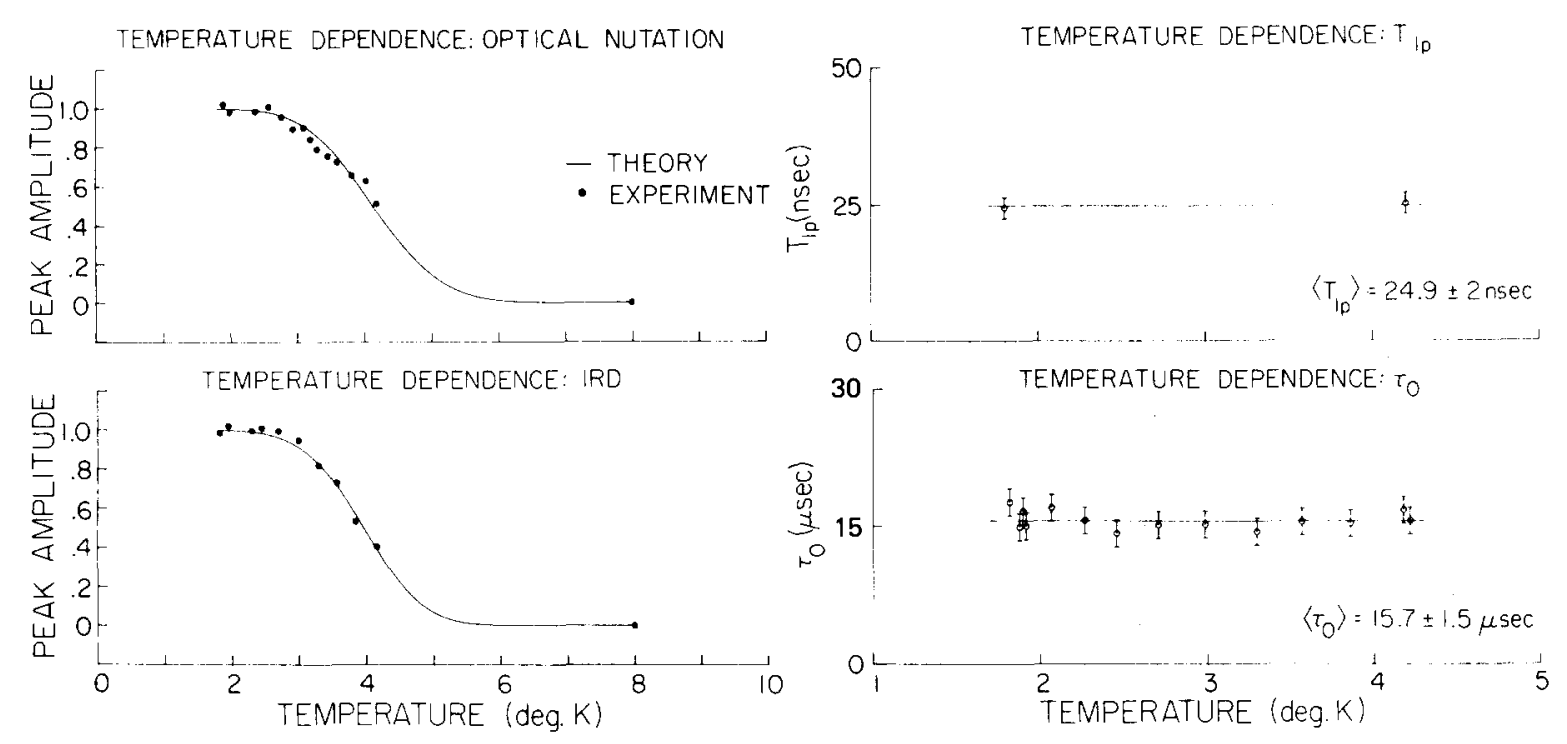

FIG. 21. The dependence of coherent transient amplitudes and lifetimes on temperature for pentacene in $p$-terphenyl. Optical nutation data for the peak amplitude fit to Eq. (29) provided values of: $\left(T_{2}\right)_{0}=44 \pm 2 \mathrm{~ns} ; \Gamma_{p}=10.4 \pm 0.3 \mathrm{GHz}$, and $\Delta=16.9 \pm 1.1 \mathrm{~cm}^{-1}$. IRD data for the peak emission intensity fit to Eq. (32) provided values of: $\left(T_{2}\right)_{0}=44 \pm 2 \mathrm{nS} ; \Gamma_{p}=12.0 \pm 0.3 \mathrm{GHz}$ and $\Delta=15.1 \pm 0.9$ $\mathrm{cm}^{-1}$. The lifetimes of both the primary level $|p\rangle, T_{1 p}$, and the slow transient decay, $\tau_{0}$, were found to be independent of temperature in the range $1.8-4.2 \mathrm{~K}$ with narrow-band excitation. Average values found were: $\left\langle T_{1 p}\right\rangle=24.9 \pm 2 \mathrm{~ns}$ and $\left\langle\tau_{0}\right\rangle=15.7$ $\pm 1.5 \mu \mathrm{s}$. The error on the $8 \mathrm{~K}$ point is quite large (see text).

ments) as a function of temperature in the range 1.8$4.2 \mathrm{~K}$ : At $1.8 \mathrm{~K} \tau_{0}=11.7 \pm 0.3 \mu \mathrm{s}, T_{1 p}=24.5 \pm 2 \mathrm{~ns}$ and at $4.2 \mathrm{~K}, \tau_{0}=12.8 \pm 0.5 \mu \mathrm{s}, T_{1 p}=25.3 \pm 2 \mathrm{~ns}$. In Fig. 21 , the temperature dependence of $\tau_{0}$, obtained from the EO results of a different crystal, and $T_{1 p}$ obtained from LADS are depicted.

Two important conclusions can now be drawn. First, the narrow-band (LADS, single-mode) and wide-band (incoherent $\mathrm{N}_{2}$ flash lamp or $\mathrm{N}_{2}$ pumped dye laser) measurements of $T_{1 p}$ give identical results. Second, the narrow-band single-mode excitation and multimode excitation give the same $T_{1 p}$ decay characteristics even though the coherence properties of the laser are different. In other words, in pentacene, the $T_{1}$ decay is insensitive to (a) the nature of the light source and (b) the environment in the temperature range studied. This situation is very different from the temperature dependence of $T_{2}$.

\section{Zeeman measurements}

Several IRD experiments were performed in the presence of a dc magnetic field parallel to the laser polarization and up to 900 Oersted in strength. Measure ments of the IRD lifetime and dc emission intensity were made on several crystals with different concentrations and orientations. A summary of data obtained in over 25 individual experiments done on different runs appears in Table III. In all cases, we observed both a decrease in dc emission intensity (up to $17 \%$ ) and a longer IRD lifetime (up to $15 \%$ ) in the presence of the field. The IRD waveforms were run at zero field, in the field, and zero field again in order to ensure that no irreversible effects were occurring (i. e. , field induced strain, etc. ). The magnitude of the effect is not that sensitive to crystal orientation but it is quite sensitive to small changes in laser frequency (i. e., one cavity mode $=390 \mathrm{MHz}$ or less). Note that because $\tau_{0}$ changes with the laser frequency one cannot at random correlate the in-field experiments with the out-of-field ones.

Since the magnitude of the effect is quite small a careful measurement of the decay over several lifetimes is necessary to accurately determine the change in "lifetime" in the presence of the field. Considering an exponential decay of the form $A(t)=A_{0} e^{-\Gamma t}$ the maximum change in intensity $\Delta A$ corresponding to a change in rate $\Delta \Gamma$ occurs at $t=\tau_{0}$ and equals $(-\Delta \Gamma / \Gamma) A$. For changes in decay rate of $10 \%-15 \%$ this represents a small change in emission intensity and even for excellent $S / N$ waveforms which we were able to obtain (typically 100-1000), several decay lifetimes were followed for all experiments to obtain accurate measurements. Possibly the overall dc signal from the detector could change partially due to the influence of a magnetic field on the path that electrons follow through the dynode chain of the photomultiplier. However, the tube is shielded and for the field strengths we used any effect of this nature would be small. Furthermore, a dc field will not affect the dynamic (time) response of the detector. The observed decay pattern therefore reflects changes in $\tau_{0}$ and also will have the largest effect at $t \simeq \tau_{0}$, as observed in the experiments.

We originally performed these experiments to determine whether the $\{|l\rangle\}$ manifold coupled to the primary level $|p\rangle$ consisted of triplets or hot ground singlets. There should be no magnetic field effect on the IRD lifetime if singlets are involved predominantly. Conversely, the participation of triplet states in the IRD decay can essentially be guaranteed if an effect is observed.

Several discussions of the rates of intersystem crossing for the individual triplet spin sublevels and the ef fect of external magnetic fields ${ }^{53-58}$ have appeared in the literature. It is generally considered with experimental evidence in some cases that fluorescence intensity mod- 
ulation occurs in the presence of a magnetic field due to changes in spin-orbit coupling strength. ${ }^{59,60}$ There should be differences in the magnitude of the effect however if the field is applied along different principal magnetic axes of the molecule since the Zeeman interaction will mix only the spin sublevels not aligned with the field. Of course, an average effect will usually be observed especially in mixed molecular crystals since different orientations of the guest exist, and the molecule in the site has a very large number of vibronic levels near $|p\rangle$.

In these Zeeman-IRD experiments the effect always resulted in a longer IRD decay time and a slightly lower dc emission intensity. The two observations are consistent in that an overall decrease in the average rate of population of $\{|l\rangle\}$ levels would decrease the overall rate of return of population to $|0\rangle$ and, therefore, reduce the dc emission intensity. The rate of population return to $|0\rangle$ from $\{|l\rangle\}$ at $\Gamma_{l 0}$ may be affected by the field also. Thus because we do not know the precise nature of the levels near $|p\rangle$ we cannot quantitatively treat the dependence of $\tau_{0}$ on the orientation and the strength of the magnetic field. Nevertheless, the experiments indicate the positive participation of spin levels in the $\{|l\rangle\}$ manifold.

\section{E. Laser scattering experiments: on- and off-resonance effects}

In a real two-level system with homogeneous broadening, the interaction between the molecular ensemble (i. e. , a collection of molecules characterized by ground and excited states) and the radiation field gives rise not only to molecular absorption and emission of light but also to the scattering of photons "off the molecule." Clearly, this scattering process depends on $T_{2}$ and the off-resonance frequency difference between the molecule and the field. Stimulated by the experimental work of Williams et al. ${ }^{61}$ on iodine, several investigators have recently addressed this question of resonance emission vs scattering ${ }^{62}$ when the molecule is excited by a weak broadband source.

Narrow -band excitation of such a system may influence the observed decay depending on the relative magnitude of the laser bandwidth and $1 / \pi T_{2}$ of the transition. ${ }^{61 \mathrm{~b}}$ In the limit where the laser bandwidth is much smaller than $1 / \pi T_{2}$, the scattering pattern will exhibit a decay which is due to the light source. This phenomenon is sometimes referred to as the Heitler effect, and has been discussed in detail by Hochstrasser and his co-workers. ${ }^{62}$

This effect was of concern to us when we studied the narrow-band transients of pentacene, since $1 / \pi T_{2}$ $=7.1 \mathrm{MHz}$ and the bandwidth of the laser on the time scale of the experiment is $\leq 3 \mathrm{MHz}$. Considering a Gaussian-shaped laser beam profile and a Lorentzianshaped molecular resonance, the response function for the interaction between the molecule and the light was calculated as a function of the ratio of the laser bandwidth to $1 / \pi T_{2}$. It was concluded that the Heitler effect is negligible, under weak excitation conditions, when this ratio becomes close to three. ${ }^{63}$ Indeed, for the narrow-band excitation of iodine, this ratio is larger than three and the decay is molecular ${ }^{63}$ as is evident from it being purely exponential and obeying ${ }^{27}$ the SternVolmer relationship.

As shown in Appendix A, the effective laser bandwidth in our narrow-band short pulse excitation experiments is not determined by the residual FM jitter $(\approx 60 \mathrm{kHz})$ of the $\mathrm{cw}$ single mode but by the Fourier transform of the pulse $(0.1-30 \mathrm{MHz})$ depending upon the pulse width. Furthermore, as the temperature is increased to $4.2 \mathrm{~K}$, there is no change in the observed decay even though $T_{2}$ has changed (see Fig. 21), and consequently the ratio of the linewidth to the laser bandwidth has been altered. Finally the "real" $T_{1}$ of the $0_{1}$ origin in these narrowband excitation experiments is the same as the $T_{1}$ observed with broadband excitation. With these facts in mind, together with the Zeeman experiments, we concluded that the effect of narrow-band scattering is not a dominant one in pentacene under our experimental conditions.

Careful investigation of the on- and off-resonance scattering can provide additional valuable information, namely ground state population dynamics. In the pentacene case, this kind of experiment is important because if the $\sim 15 \mu \mathrm{s}$ decay represents a population flow from $|p\rangle$-to- $\{|l\rangle\}$-to- $|0\rangle$, then one should observe a slow transient on the scattering signal that characterizes in part the time dependence of the ground state population.

To check the above hypothesis we performed resonance and off-resonance laser scattering experiments in the mixed crystal at $1.8 \mathrm{~K}$. Since $|0\rangle$ receives population from the slow decay channel while $|p\rangle$ loses population to the slow channel, one might naively think at first that the laser scattering transients should provide the recovery time of the ground state and thus show a buildup transient rather than the decay seen in emission (Fig. 19). This is not a correct picture of the population flow within the level structure, however, for light on experiments. In the presence of the laser field, net population is continuously lost from both $|0\rangle$ ) and $|p\rangle$ (at a rate $\left.\tau_{0}^{-1}\right)$ to the $\{|l\rangle\}$ manifold until $\Gamma_{p l} \rho_{p p}=\Gamma_{l 0} \rho_{l l}$ and equilibrium exists. Thus, true molecular scattering in pentacene should produce a scattering transient that decreases in intensity during the laser pulse, as shown in Fig. 22.

The procedure used in these experiments was as follows. During a single-mode laser pulse (LADS method), scattered laser light from the sample was collected at right angles to the laser beam and was observed through a $0.5 \mathrm{~m}$ Jarrel-Ash spectrometer with narrow slits $\left(0.7 \mathrm{~cm}^{-1}\right)$. The experiments were performed with the laser on-and off-resonance with the $0_{1}$ origin. Although the sample emits at the laser frequency, most of the emission (Fig. 14) occurs to upper vibronic levels of the ground state and with narrow slits the scattered laser light signal dominates. The scattering transient was also observed by de Vries et al. ${ }^{52 b}$ but no quantitative analysis of the data was provided.

The laser scattering results (Fig. 20) are compared with theory in Fig. 22 together with the LADS emission decay at identical pulse width and laser power. As one can see, the emission signal decays by a slow transient. However, the on-resonance scattered laser signal shows a slow buildup in intensity (Fig. 20) instead of the 
LEVEL POPULATION: STEADY-STATE COHERENCE REGIME PENTACENE, $1.8 \mathrm{~K}$

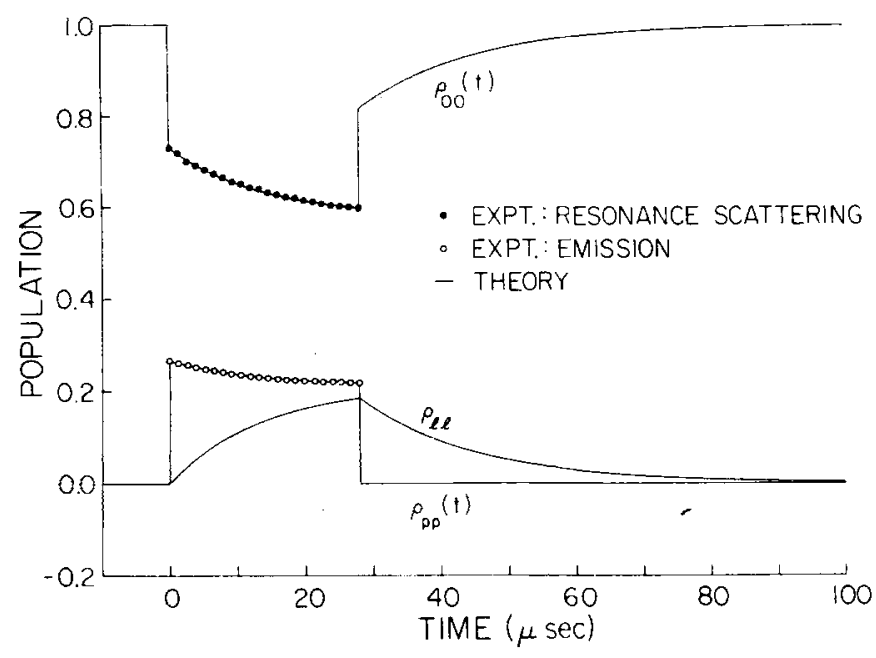

FIG. 22. Shown here plots of the population of $|0\rangle,|p\rangle$, and $|l\rangle$ obtained from the density matrix equations in the steadystate coherence regime [Eqs. (43) and (48)], and experimental data points from the resonance scattering and LADS emission transients (see Fig. 20) at $1.8 \mathrm{~K}$. The observed decay rate for $\rho_{\text {po }}(t)$ is identical to the buildup rate of resonance scattering intensity. The best fit value for both is $13.7 \pm 0.4 \mu \mathrm{s}$. Resonance scattering data has been plotted on $\rho_{00}(t)$ to show that it corresponds to a depletion of population from the ground state.

decay predicted by the time dependence of the ground state population shown in Fig. 22. This buildup rate is identical with the ground state population decay rate as shown by the excellent fit of experimental scattering data to theoretical ground state population as a function of time. (Fig. 22).

From the experiments of Rousseau and Williams ${ }^{61}$ on $I_{2}$ one knows that following a laser pulse excitation onresonance, the lifetime of the molecular scattering process is governed by the natural (spontaneous) lifetime of the excited state while off-resonance, the scattering lifetime is determined by the excitation pulse characteristics. During the excitation pulse, however, the time dependence of the scattering intensity should reflect the approach of $|0\rangle$ and $|p\rangle$ towards equilibrium. If the background scattering of the laser beam (due to crystal imperfections, etc.), i. e., the off-resonance scattering of Fig. 20, is much larger than molecular resonance scattering then the observed scattering signal (on-resonance) will show a slow buildup transient due to self-absorption effects rather than the slow decay due to molecular scattering. In our experiments, background scattering is strong and thus the observed buildup characterizes the time dependence of self-absorption in the sample. One knows that the absorption coefficient is proportional to the population difference between the levels involved in the absorption process. Using Eqs. (43) and (48) of the density matrix, the population difference between $|0\rangle$ and $|p\rangle$ can be determined. One obtains an expression that decays exponentially (following the initial jump; Fig. 22) at a rate given by $\tau_{0}^{-1}$ towards an equilibrium value of $\left(\Gamma_{p t}+\Gamma_{p 0}\right) \Gamma_{t 0} /$ $\lambda_{+} \lambda_{\text {. }}$. Since the amount of background scattered laser light reaching the detector increases as the absorption decreases, the observed buildup in the laser scattering signal is consistent with a decrease in the absorption of the sample as population is lost to the slow decay channel. This interpretation is supported by (1) the fact that the absolute scattering intensity increases offresonance with no buildup or decay ${ }^{64}$ and (2) this same effect is seen at much lower concentration $\left(1 \times 10^{-8} \mathrm{~m} /\right.$ $\mathrm{m}$ ) where although absorption is lower, total molecular density is lower also. Thus the effect we see here at the 0,0 frequency is not an artifact related to the sample concentration. Furthermore, background scattered laser light need not be of concern in emission experiments since laser light is blocked with appropriate sharp-cut filters at the detector and the relatively intense emission to upper vibronic levels of the ground state is observed exclusively.

These laser scattering experiments agree with the results obtained from emission experiments concerning the origin of the slow transient decay and are consis tent with the level structure shown in Fig. 4 for pentacene.

\section{F. Effect of temperature on dephasing}

\section{Theoretical}

As shown in Fig. 21, the pure dephasing rate of pentacene, which forms an impurity in the $p$-terphenyl host, depends on the lattice temperature in a different manner from that of $T_{1}$ processes. The interesting questions are therefore (a) why is this true in solids and (b) can we describe this distinct behavior and fit the experimental results without yielding to a phenomenologi cal approach?

Several models are now known to give rise to a temperature dependent linewidth and frequency shift. The models have been used to describe vibrational dephasing in liquids and also some molecules in rigid media.

Diestler ${ }^{65}$ has recently reviewed vibrational phase and population relaxation theory as applied to a harmonic oscillator in a heat bath. The work of Fischer and Laubereau, ${ }^{66}$ Rice and Oxtoby, ${ }^{67}$ and Madden and Lynden-Bell ${ }^{68}$ have emphasized the role of $T_{2}$ and $T_{1}$ in determining the observed vibrational dephasing time. Oxtoby ${ }^{69}$ has shown that an accurate $T_{2}$ calculation for $\mathrm{N}_{2}$ can be obtained by means of a molecular dynamics simulation. More recently, Harris and co-workers ${ }^{70}$ have advanced an exchange model based on the KuboAnderson ${ }^{71}$ theory to describe the effect of temperature on $T_{2}$ and on the line shift $\delta$. The exchange model requires the presence of low frequency molecular or Iocalized phonons (on the impurity molecule) that undergo changes in frequency on going to the excited state.

Thus, it is expected that the temperature dependence of the dephasing will be determined by the thermalization process (like $e^{-\Delta / k T}$, where $\Delta$ is the phonon energy) into the nearby level.

This "activation" energy expression also appears to explain the temperature dependence of $T_{2}$ obtained from photon echo experiments done on optical transitions. Thus we are now faced with an interesting point; if exchange is the dominant process for the destruction of phase coherence, then there must be "selective" modes by which dephasing occurs. Furthermore, in the ex- 
change model the role of acoustic phonons is incorporated only indirectly, even though the experiments of interest are usually carried out at temperatures below the Debye cutoff.

In what follows we highlight a theory developed by Jones and Zewail ${ }^{72}$ for optical dephasing in condensed phases as applied to pentacene. The expressions obtained for dephasing in terms of molecular scattering processes were originally derived by Jones et al. ${ }^{73}$ utilizing the optical theorem of scattering theory and the Redfield's superoperator technique exploited by Snider ${ }^{74}$ and recently by Liu and Marcus ${ }^{75}$ for describing phase relaxation in a two-level system. We have obtained ${ }^{72}$ these same expressions more recently by using the projection operator technique of $\mathrm{Zwanzig},{ }^{76}$ which has been applied to line shape problems (especially in gases) in the classical work of Fano ${ }^{77}$ and Ben-Reuven. ${ }^{78}$ In this way, it was possible to cast the parameters of dephas ing in solids as a function of temperature, and it was also possible to include the exchange terms in a nonphenomenological way. The theory (to lowest order) has been applied primarily by Diestler ${ }^{79}$ to liquids. With reasonable models, the theory gives approximate $T_{2}$ 's and provides their temperature dependence. For more details on the treatment the reader is referred to Ref. 72.

Theoretically, the molecule is treated as consisting of a single resonance with initial $(i)$ and final $(f)$ states interacting with a bath without discrimination between optical and acoustic modes. The Hamiltonian describing this system is

$$
\mathfrak{H}=\mathfrak{K}_{m}+\mathfrak{H}_{\not \mathrm{h}}+V \text {, }
$$

where $\mathfrak{H}_{m}$ is the molecular impurity (pentacene) Hamiltonian, $\mathcal{H}_{p h}$ the phonon Hamiltonian and $V$ the interaction term. Thus, we have

$$
\begin{aligned}
& \mathfrak{H}_{m}=\frac{1}{2}\left(\begin{array}{cc}
\hbar \omega_{0} & 0 \\
0 & -\hbar \omega_{0}
\end{array}\right)=\left(\begin{array}{cc}
E_{p} & 0 \\
0 & E_{0}
\end{array}\right) \\
& \mathfrak{H}_{p \mathrm{ph}}=\sum_{a j} \hbar \omega_{q j}\left(a_{q j}^{+} a_{q j}+\frac{1}{2}\right) \\
& V=V_{1} S+V_{2} S^{2}+\cdots \\
& S=i \sum_{q j}\left(\frac{\hbar}{2 M \omega_{q j}}\right)^{1 / 2}(q j)\left(a_{q j}-a_{q j}^{+}\right) .
\end{aligned}
$$

Here, $V_{1}$ and $V_{2}$ are the coupling parameters between the molecule and the phonons, and $S$ is a strain operator which creates $\left(a^{+}\right)$or destructs $(a)$ a phonon (wave vector $q$ and mode index $j$ ) as a result of the tensorial distortion of the lattice around the excited molecule. $a^{+} a$ is the occupation number in the reservoir, and $M$ is the mass.

The total dephasing rate for a system described by the above Hamiltonian is ${ }^{72}$ :

$$
\begin{aligned}
\frac{1}{T_{2}}= & \frac{1}{2}\left(\frac{1}{T_{10}}+\frac{1}{T_{1 p}}\right) \\
& +\frac{\pi}{\hbar} \sum_{q, q^{\prime}} W_{p}\left|\left\langle 0 q^{\prime}|T| 0 q\right\rangle-\left\langle p q^{\prime}|T| p q\right\rangle\right|^{2} \delta\left(E_{q}-E_{q^{\prime}}\right) \\
= & \frac{1}{2}\left(\frac{1}{T_{10}}+\frac{1}{T_{1 p}}\right)+\frac{1}{T_{2}^{\prime}},
\end{aligned}
$$

where, the initial and final states are 0 and $p$, respectively, and $T_{2}^{\prime}$ is the pure dephasing time. The temperature dependence of $T_{2}^{\prime}$ is now contained in the $\sum W_{p}$ which depends on the density of bath states and the phonon thermal distribution. On the other hand, the transition $(T)$-matrix element difference between the initial and final states which now has the phonon states as well ( $q j$ the phonons are replaced by $q$ for the sake of simplicity) determines the source of the elastic scattering "anisotropy" between the two states of the transition. In other words, if this anisotropy is large then the cross section for the $T_{2}^{\prime}$ process will be relatively large when compared with the cross section for the $T_{1}$ process. As we shall see from our data on pentacene, the anisotropy term temperature dependence dominates that of the $T_{1}$ term simply because the cross section of the former is very large for optical transitions. ${ }^{79}$

We shall assume that for pentacene in $p$-terphenyl the lowest order contributions to $T$ are the important ones. These processes include two-phonon scattering and can be handled if we expand $T$ in terms of $V$ using Dyson's equation:

$$
T=V+V G_{0} T=V+V G_{0} V+\cdots,
$$

where $G_{0}$ is the free propagator (Green operator). It is apparent that the two-phonon processes originate from the first order term of the strain expansion of $V$ carried to second order in the Dyson series or the secondorder term of the strain expansion carried to first order in the Dyson series. However, we note that the former contribution will be approximately on the order of the population decay rate and will be neglected in the case of interest.

Combining Eqs. (51)-(53), we have

$$
\begin{aligned}
\frac{1}{T_{2}^{\prime}}= & \frac{4 \pi}{\hbar} \sum_{q, q^{\prime}}\left(\frac{\hbar q^{2}}{2 M \omega_{q}}\right)^{2}\left\langle n_{q}\right\rangle\left\langle n_{q^{\prime}}+1\right\rangle \\
& \times\left|\left\langle\Delta V_{2}\right\rangle_{p 0}\right|^{2} \delta\left(E_{q}-E_{q^{\prime}}\right),
\end{aligned}
$$

where the brackets, describing phonon absorption and emission, denote thermal averages and $\Delta V_{2}=\left\langle p\left|V_{2}\right| p\right\rangle$ $-\left\langle 0\left|V_{2}\right| 0\right\rangle$. For acoustic phonons obeying the Debye model, the density of states is quadratic in frequency, and is only nonzero up to the cutoff frequency $\omega_{D}$. Using the expression for the Debye density of states function and Eq. (54) yields,

$$
\frac{1}{T_{2}^{\prime}}=\text { constant }\left|\left\langle\Delta V_{2}\right\rangle_{p 0}\right|^{2}\left(\frac{k T}{\hbar}\right)^{7} \int_{0}^{x_{d}} \frac{X^{6} e^{X} d x}{\left(e^{X}-1\right)^{2}},
$$

where $X_{D}=T_{D} / T$. Thus for temperatures much less than the Debye temperature, the rate of pure optical dephasing is

$$
\frac{1}{T_{2}^{\prime}}=\text { const }\left|\left\langle\Delta V_{2}\right\rangle_{p 0}\right|^{2} T^{7}
$$

and we now recover the $T^{7}$ term. We note that this is the same as the $T^{7}$ that describes linewidths determined by Raman processes, discussed in Ref. 72 and in the literature by many other authors. ${ }^{80}$ One can see, however that the coefficient (anisotropy term) in front of $T^{7}$ is what makes the pure dephasing term a dominant one in a way similar to elastic dephasing in gases under the binary collision approximation. Two interesting points can now be made. First, Eq. (56) is similar to the one used by Diestler ${ }^{79}$ for describing dephasing of $\mathrm{N}_{2}$ in $\mathrm{Ar}$ 
using the Born approximation method. Second, the other temperature terms of acoustic phonons can be obtained from Eq. (52) without (yet) considering the mechanism of dephasing by exchange into other states. For example, if the phonon branch has an effective mode (e. g. , quasilocalized or resonance ${ }^{81}$ ) of frequency $\Delta / \hbar$ then we obtain the Orbach-type term using the same prescription given above, but with a different cross section. To illustrate this point more clearly let us consider the case of a resonance mode that causes dephasing. This case is particularly useful for the pentacene $/ p$-terphenyl system.

Dephasing by optical phonons is different from that which occurs by acoustic phonons. The difference arises since the "optic" phonon is described by essentially one en ergy, $\hbar \omega_{0 p}=\Delta$. If one assumes that the density of phonon states for this mode (optical or "resonance") is sharply peaked about $\Delta$, then the temperature dependence is easy to ascertain [Eq. (54)]:

$$
\begin{aligned}
\frac{1}{T_{2}^{\prime}} & =\text { constant } \frac{\exp (\Delta / k T)}{[\exp (\Delta / k T)-1]^{2}}\left|\left\langle\Delta V_{2}\right\rangle_{p 0}\right|^{2} \\
& =\Gamma_{p} \exp (-\Delta / k T) ; \quad \Delta \gg k T,
\end{aligned}
$$

where $\Gamma_{p}$ denotes a pre-exponential constant. This result provides several interesting points: (a) it applies to pentacene nicely; (b) it is derived from formal theory, and thus the comparison with experiments is not phenomenological; (c) it described the Orbach mechanism (which is similar in form to the temperature dependence results of the exchange model) without invoking any extra levels. As discussed in Ref. 72, exchange comes from inter resonance coupling, i. e., off-the-energy shell terms in the language of scattering theory.

Finally, the frequency shift $\delta$ of the transition can also be deduced as a function of temperature in the same manner. The shift is given ${ }^{72}$ by the sum of two terms: $\delta_{1}$ which includes energy shifts of 0 and $p$ (of the form $\left.|\langle T\rangle|^{2} / \Delta_{q q^{\prime}}\right)$ similar in meaning ${ }^{80}$ to the $T_{1}$ term of Eq. (52), and $\delta^{\prime}$ which contains an anisotropy term similar to that of $T_{2}^{\prime}$ and is given by

$\delta^{\prime}=\frac{\pi}{\hbar} \operatorname{Re}\left\{i \sum_{\alpha, \sigma^{\prime}} W_{Q}\left\langle\Delta T^{-}\right\rangle_{0 p}\left\langle\Delta T^{*}\right\rangle_{\psi_{p}} \delta\left(E_{\alpha}-E_{\alpha^{\prime}}\right)\right\}$,

where

$$
\left\langle\Delta T^{ \pm}\right\rangle_{0 p}=\left\langle 0 q|T| 0 q^{\prime}\right\rangle_{ \pm}\left\langle p q \downarrow T \mid p q^{\prime}\right\rangle .
$$

It is interesting to note that because the temperature dependence is determined by the density of states of the phonons, the $\delta^{\prime}$ calculation for both kinds of phonons gives a temperature dependence function like that obtained for the linewidth. ${ }^{81 \mathrm{~b}}$ Physically, this makes sense as the same processes are responsible for the shift and the linewidth - an anisotropy in the scattering amplitudes for the two states of the transition. Thus we conclude that the ratio of the linewidth to the shift is generally expected to be temperature independent, and that the use of this criterion to invoke the exchange model is not unequivocal. Furthermore, the expression for $\delta^{\prime}$ clearly indicates that the shift could be either positive or negative as observed experimentally, but the physics that determines this behavior is now more clear - the anisotropy of the scattering matrix element between the ground and the excited states. Thus for electronic transitions the shift is expected to be one way, while for the IR transitions it might go either way. Also of significance, Eq. (52) indicates that some electronic transitions may exhibit faster dephasing than IR or spin transitions. 79,82

\section{Nutation, IRD, and echo resu/ts}

As mentioned before, the data presented in Fig. 21 show the distinct behavior for $T_{2}^{\prime}$ and $T_{1}$-type processes as a function of temperature. This distinction comes about because of Eq. (52). For the spontaneous lifetime of the $|p\rangle$ state in pentacene, we found no difference between the 1.8 and the $4.2^{\circ} \mathrm{K}$ results $(24.9 \pm 2 \mathrm{~ns})$. Similarly, there is no dependence on temperature for $\tau_{0}$ in the range $1.8-4.2 \mathrm{~K}^{83}$; i. e., $15.7 \pm 1.5 \mu \mathrm{s}$. These two observations indicate that the population loss rate of the $|p\rangle$ state is not sensitive to temperatures (1.8-4.2 K) and that the pure dephasing rate, which reflects differences in the scattering amplitude of excited and ground states [Eq. (52)], is changing with temperature by acoustic and/or optical phonons of the mixed crystal, i. e. , including resonance and quasilocalized modes.

If the impurity (pentacene) is dephased by phonons within the entire frequency distribution of the acoustic branch, then a $T^{?}$ temperature dependence, Eq. (56), for $T_{2}^{\prime}$ is expected. Thus, one would expect $1 / T_{2}$ to be constant in the low temperature regime and increase when the $T^{7}$ process becomes active. On the other hand, a resonant phonon mode built on the electronic ground and/or excited states can make the impurity dephase according to Eq. (57), and the optical resonance will shift following Eq. (58).

From the nutation and the IRD results of Fig. 21, we obtained the following parameters from the computer fit and using Eq. (57):

$$
\begin{array}{ll}
\text { Nutation: } & \Delta=16.9 \pm 1.1 \mathrm{~cm}^{-1} ;\left(T_{2}\right)_{0}=44 \pm 2 \mathrm{~ns} \\
& \text { and } \Gamma_{p}=10.14 \pm 0.3 \mathrm{GHz} \\
\text { IRD: } & \Delta=15.1 \pm 0.9 \mathrm{~cm}^{-1} ;\left(T_{2}\right)_{0}=44 \pm 2 \mathrm{~ns} \\
& \text { and } \Gamma_{p}=12 \pm 0.3 \mathrm{GHz} .
\end{array}
$$

The above values for $\left(T_{2}\right)_{0}$, i. e., $T_{2}$ at very low temperatures $(0$ means zero degree which in our case implies $1.8 \mathrm{~K}$ ) is in very good agreement with the OFID and echo ${ }^{49}$ results $(44 \pm 3 \mathrm{~ns})$. What is not in good agreement is $\Delta$ which is slightly smaller ( 17 as compared to $21 \mathrm{~cm}^{-1}$ ), and $\Gamma_{p}$. The difference is not that serious, however, since both of the values are obtained by fitting the data with two parameters, but we also know that the lack of data between 4.2 and $8 \mathrm{~K}$ introduces some error. The important point is that all of the available results on pentacene are consistent in that they show agreement on the measured $T_{2}$ and $T_{1}$ and on the transition temperature $(3.7 \mathrm{~K})$ at which $T_{2}^{\prime}$ processes change with temper ature. ${ }^{84}$ It is interesting to note that the $\Delta$ measured for two of the higher energy sites is $18.7 \mathrm{~cm}^{-1}$ from the echo results. ${ }^{49}$

With these data, we conclude that there is an effective intermediate state that dephases the impurity, although at the moment we cannot exclude all other channels discussed in Sec. VF1. The intermediate is separated by 
$\sim 17 \mathrm{~cm}^{-1}$ from the guest states. In terms of the two states involved in the optical transition of pentacene $\left({ }^{1} A_{1 g}\right.$ and $\left.{ }^{1} B_{2 u}\right)$, the $17 \mathrm{~cm}^{-1}$ state could be either a ground or excited phonon state. Neither the absorption spectra of our crystals nor the crystals of Wiersma et al. ${ }^{49}$ have shown a peaked phonon sideband at this frequency. Based on the fact that pentacene in $p$-terphenyl does not exhibit these resonance modes on the side band while tetracene does (at $\sim 7 \mathrm{~cm}^{-1}$ ), Wiersma et al. ${ }^{49}$ concluded that the guest and not the host is "introducing" the for mation of these resonance modes. To us the origin of these modes cannot be established with the few experiments available. The theory presented here can explain the absence of these peaked sidebands by allowing the distribution width of the density of states to be relatively wide. If the mode is a resonant one, its absence in the sideband of the terphenyl host doped with pentacene implies that it has the same frequency in the ground and excited state to within the inhomogeneous linewidth.

The values of $T_{2}$ obtained above at $1.8 \mathrm{~K}$ when compared with $T_{1 p}(24.9 \mathrm{~ns})$ indicate that $T_{2}=2 T_{1 p}$, to with in $12 \%$. The experimental errors are typically $4 \%$ and thus the difference may be due to self absorption (note that $\boldsymbol{\mu} \sim 1 D$ ) and/or some intrinsic intersite effects. In very dilute crystals, $T_{1 p}$ reaches 23.5 ns suggesting the decrease in radiation trapping that is expected when the crystal absorbs the light strongly. Note that reabsorption will influence $T_{1}$ while echo absorption will lead into a secondary echo ${ }^{85}$ or $T_{1}$ spontaneous decay. It is perhaps worth mentioning that, as shown in Fig. 13, even when we excite $0_{1}$ solely with the laser, $0_{4}$ emission appears with a relative intensity of $\sim 3 \%$. If $\mathbf{0}_{4}$ is acting as an intermediate dephasing level, it will contribute to the overall $T_{2}$ measurement.

The significance of $\Gamma_{p}$ is now evident from Eqs. (56) and (57). It is a pre-exponential describing the difference in the scattering amplitudes of $|0\rangle$ and $|p\rangle$ due to the interactions with $q$ and $q^{\prime}$. It is not clear to us why this should be called a lifetime of the phonon. ${ }^{49}$ It is a lifetime if one is dealing with pure $T_{1}$ processes (kinetic approach) butfor $T_{2}^{\prime}$, "scattering" events are the processes involved. More on this point will be published by Jones and Zewail elsewhere.

Finally, the value of $T_{2}$ obtained using the results in Fig. 21 at $4.2 \mathrm{~K}$ is consistent with the fact that at this temperature we still see IRD, and therefore the homogeneous width must be $\leqslant 60 \mathrm{MHz}$, the maximum switched frequency of the laser. Thus, one can use these data to predict $T_{2}$ at higher temperatures. Of course, if the temperature is high enough to give say $T_{z}$ of $10 \mathrm{ps}$, then the width becomes $1.1 \mathrm{~cm}^{-1}$ and one might therefore measure it directly without elaborate picosecond methods.

\section{G. Effect of inhomogeneous broadening}

In describing the population flow in pentacene at long times, we have used the solution of $\rho$ on resonance, and showed that the results agree with experiments quite well. In this section we discuss the effect of inhomogeneous broadening and justify the neglect of its averaging effects in the high-power limit.

Equation $(41)$ is the on-resonance solution for $\rho_{p p}(t)$ obtained by solving the density matrix Eq. (39b) appropriate for the level structure of pentacene. To consider the effect of inhomogeneous line shape averaging on the population dynamics, one must retain the off-resonance term of the pumping rate [i. e., $\left.R_{p}=\frac{1}{2} \chi^{2}\left(\Gamma / \Gamma^{2}+\Delta^{2}\right)\right]$, and average Eq. (41) over $g(\Delta)$. Since the only time dependence of $R_{p}$ is from the known laser pulse characteristics [see pulse $A$ of Fig. 24 and Eq. (45)], Eq. (41) can be solved for $\rho_{p p}(t)$ as shown in Eq. (43) and then averaged over the line shape. This averaging is, in general, important because it shows that the hole burned in the inhomogeneous line will saturate as $R_{p}$ increases. Hence in a time resolved experiment where $R_{p}$ changes indirectly the number of pumped molecules will change.

Equation (43) contains $R_{p}$ and thus $\Delta$ in both the coef ficients and arguments of the exponentials. Therefore, averaging of Eq. (43) in this form would have to be done numerically. However, in the high power case where $R_{p}$ is greater than the relaxation rates, Eq. (43) may be simplified by a binomial expansion of the squa re root expression (for large $R_{p}$ ) in Eq. (44). This is valid in our experiments where (1) an overshoot in the emission intensity ( $a$ in Fig. 8) occurs at all laser powers used, indicating that $\rho_{p p}(t)$ is initially driven beyond its final equilibrium value, and (2) the observed decay time, $\tau_{0}$, is independent of laser power which only oceurs in the high power case. The expansion shows that:

$$
\lambda_{\leftarrow} \simeq-\left(\frac{2 \Gamma_{l 0}+\Gamma_{p l}}{2}\right)=-\tau_{0}^{-1}
$$

and

$$
\lambda_{-} \simeq-2 R_{p} \text {. }
$$

The final form of Eq. (43) then becomes:

$$
\begin{aligned}
\rho_{p \rho}(t) \simeq & \frac{1}{2}\left[\left\{\frac{\Gamma_{p l}}{2 \Gamma_{l 0}+\Gamma_{p l}}\right\} \exp \left(-t / \tau_{0}\right)-\exp \left(-2 R_{p} t\right)\right] \\
& +\frac{\Gamma_{l 0}}{2 \Gamma_{l 0}+\Gamma_{p l}} .
\end{aligned}
$$

Thus, in the high power case only the buildup rate for $\rho_{p p}(t)$ needs to be averaged, a great simplification over the numerical integration which does not provide physical insight into the problem. The observed decay time $\tau_{0}$ is completely determined by the magnitude of the rate constants $\Gamma_{t 0}$ and $\Gamma_{p l}$, and will not be affected by averaging over the inhomogeneous line shape. At this point we should evaluate the fraction of the total inhomogeneous line pumped in the high power case and determine if Eq. (60) is valid. This can be done by calculating the magnitude of the off-resonance parameter $\Delta$ required to reduce $R_{p}$ from the pumping rate obtained in nutation experiments (at the same power) to an effective value where the binomial expansion of the square root expres sion [Eq. (44)] is invalid (i. e., $R_{p} \simeq \Gamma_{p 0}$ ). This calculation reveals that Eq. $(60)$ is valid for the entire power broadened linewidth $\left(\delta \omega_{P B}\right)$ since $R_{p}>\Gamma_{p 0}$ for $\Delta \leq \pm 120$ $\mathrm{MHz}$. Therefore, we can average $\exp \left(-2 R_{p} t\right)$ in Eq. (60) over $\delta \omega_{P B}$ or $\delta \omega_{I}$ (the molecules pumped by the laser only span $\delta \omega_{P B}$ ) to obtain an effective pumping rate:

$\left\langle\exp \left(-2 R_{p} t\right)\right\rangle=\left\langle\exp \left(\frac{-\chi^{2} \Gamma t}{\Gamma^{2}+\Delta^{2}}-\frac{\Delta^{2}}{\delta \omega_{P B}^{2}}\right)\right\rangle$ 


$$
\simeq\left\langle\exp \left(\frac{-\chi^{2} \Gamma t}{\Delta^{2}}-\frac{\Delta^{2}}{\delta \omega_{P B}^{2}}\right)\right\rangle=\exp \left(-2 R_{p_{\text {ef }} t} t\right),
$$

where

$$
R_{p_{\text {eff }}}=\left(\chi / \delta \omega_{P B}\right) \sqrt{\Gamma / t} .
$$

This approximate solution (see Appendix B) shows that averaging the expression for the buildup rate essentially dilutes $R_{p}$ by the power-broadened linewidth. Using the results of Eq. (61) and Eq. (45) which describes the exponential turn on of the laser pulse, we obtain an expression that agrees quite well with the observed emission intensity buildup rate.

Two important conclusions can be drawn from the above discussion. First, in the high power case, inhomogeneous line shape averaging of $\rho_{p p}(t)$ has essentially no effect on the observed transient decay time and, second, averaging of the buildup expression dilutes the pumping rate by the power-broadened linewidth, ef fectively. The effect of laser power on the observed transient patterns is now perhaps physically understood.

It is also clear now that by using Eqs. (60) and (61) from the high power limit and by knowing $\gamma$ and $\tau_{0}$ experimentally, one is able to determine unambiguously $\Gamma_{p l}$ and $\Gamma_{l 0}$, within the framework of the above approximations and especially if they are very different. This leads to the following useful formula relating $\gamma$ to $\tau_{0}$ :

$$
\tau_{0}^{-1}=\frac{1}{2} \Gamma_{p l}\left(1+\gamma^{-1}\right)
$$

Thus in the high power limit, one obtains $\Gamma_{p l}$. We shall use these results in the coming section to help us identify the nature of $|l\rangle$.

\section{H. On the structure of $\{\mid n\}$}

The structure of the $\{|l\rangle\}$ manifold will be ascertained from more than ten independent experiments. Several of these were reported in $I$ and have been repeated again in this work. The following summarizes all that we have observed:

(a) The observation of a $15 \mu$ s decay when the emission was monitored at right angles to the exciting laser beam and the EO method was used with long time pulses $(50-100 \mu \mathrm{s})$.

(b) A Zeeman effect was observed on both the dc and the decay (see Table III).

(c) As the temperature increases the peak of the IRD decreases. time.

(d) $T_{2}(44 \mathrm{~ns})$ is much smaller than the $15 \mu \mathrm{s}$ decay

(e) The decay signal $(15 \mu \mathrm{s})$ is absent when the molecule is excited above its electronic origin.

(f) The decay time does not change with changes in the laser power $(\sim 1-50 \mathrm{~mW})$, but the buildup rate does.

(g) The decay is exponential and changes as we scan the narrow-band laser within the inhomogeneous $0_{1}$ origin.

(h) The signal gets stronger as we increase the switching frequency, i.e., getting further away from the homogeneous width. (i) Exciting with a long time single pulse (LADS and not the EO) the $15 \mu \mathrm{s}$ decay is only observed during the light-on cycle. This observation which we reported earlier ${ }^{51}$ was also made independently by de Vries et al. ${ }^{52 \mathrm{c}}$

(j) Although the inhomogeneous broadening of all sites are comparable in width $\left(0.7-3 \mathrm{~cm}^{-1}\right.$ depending on the concentration) $T_{1}$ and $T_{2}$ of the sites are different.

(k) Exciting with a single-mode laser, a multimode laser or an incoherent source gives the same $T_{1 p}$ decay time for the $0_{1}$ origin. The lifetimes of $0_{1}$ and $0_{2}$ are the same at 1.8 and $4.2 \mathrm{~K}$.

(1) Exciting into the $267 \mathrm{~cm}^{-1}$ mode of $0_{1}$, neither LADS transients nor EO transients have been observed. However, the falling edge of a LADS signal still gives the same value for $T_{1 p}$.

(m) Exciting with the single mode into the 0,0 of $0_{1}$ and monitoring the light into the 0,0 (scattering and emission) or into higher vibrational ground states (emission) gives identical decay times but different transient patterns (Figs. 20 and 22).

Observations (a)-(h) lead us to conclude that the narrow-band excitation prepares molecular eigenstates that have much longer lifetimes, consistent with the prediction of Eq. (1). However, if this were true then (i) should show the $15 \mu \mathrm{s}$ decay on the falling edge of the LADS pulse. Following the observation (i) we concluded that it is possible that we have prepared a primary singlet state that decays into states that do not contain much of the singlet oscillator strength or that we just simply prepared $|p\rangle .{ }^{51}$ From all the observations, (i) $-(m)$ and also $(a)-(h)$, we revise the early interpretation of I and conclude the following: The state $|p\rangle$ is excited very rapidly since $\chi$ (the pumping rate) is very large. Then during a short time light pulse coherence effects between $|0\rangle$ and $|p\rangle$ are manifested. During a long time pulse, however, the excitation (7 MHz width) is statistically going into $|l\rangle$, which as shown late $r$ is consistent with being a triplet manifold. The key parameter here is the decay time on the falling edge of a LADS pulse which gives a $T_{1}$ decay time that is identical to $T_{1}$ obtained from the broad band and incoherent excitations.

The interesting question now is what is the relationship between the structure of $|l\rangle$ and the long decay transient observed during the long-time pulse? Several possibilities exist, but we shall rule out all (that we can think of) but one.

The first possibility is that there is a slow intersystem crossing (ISC) from $|p\rangle$ to resonant or near-resonant $l$ states (built on the origin of the $T_{1}$ state) followed by fast vibrational relaxation (as in the singlet manifold) and then decay from the triplet origin to $|0\rangle$. The second possibility is a fast decay from $|p\rangle$ into resonant $l$ states (either hot vibrational levels of $T_{1}$ or a new state like $T_{2}$ ) followed by slow vibrational relaxation or electronic relaxation, to the bottom of $T_{1}$, and then decay from $T_{1}$ to $|0\rangle$ by radiative and nonradiative channels. A third possibility is a fast decay from $|p\rangle$ into $l$, a fast decay from resonant $l$ to $T_{1}$ and a slow decay from $T_{1}$ to the ground state. 
Several points support the slow depletion out of $|p\rangle$. First, although it will not be surprising to us if vibrational relaxation is slow at $1.8 \mathrm{~K}$ for certain modes ${ }^{86}$ in large molecules, it will be difficult to accept that vibrational relaxation is fast in the singlet and slow in triplet manifolds. Furthermore, as shown by Lim and his coworkers, ${ }^{87}$ and by others, the internal conversion rate between triplets $\left(T^{\prime}-T\right)$ in anthracenes is typically 2 $\times 10^{10} \mathrm{~s}^{-1}$ while the radiative rate for $T_{2} \rightarrow T_{1}$ is typically $2 \times 10^{5} \mathrm{~s}^{-1}$. The energy gap between $T_{2}$ and $T_{1}$ in anthracene is $\sim 11200 \mathrm{~cm}^{-1}$. In general, as the gap gets smaller, there seems to be a trend in large molecules in that the rate of internal conversion increases (energy gap low) except at very low energy gaps $\left(<2000 \mathrm{~cm}^{-1}\right)$ where it decreases again. ${ }^{88}$ As in anthracenes, we do not know how many triplets are involved in the ISC. What can be inferred from the experiments, however, is that ISC in pentacene is slow and not $\sim 10^{8} \mathrm{~s}^{-1}$ as in other aromatic hydrocarbons, in which a determination has been made, with the exception of pyrene (ISC rate constant $\left.=3 \times 10^{4} \mathrm{~s}^{-1}\right)$. This point can be shown using Eq. (62).

Since we know $\gamma$ and $\tau_{0}$ experimentally for pentacene, Eq. (62) in the high power limit dictates that $\Gamma_{p l}$ be at hand. In fact, if the overshoot is very large, $\gamma^{-1} \rightarrow 0$, then $\Gamma_{p l}=2 / \tau_{0}$. For measured values of $\tau_{0}=19.6 \mu \mathrm{s}$ and $\gamma=0.9$ in an EO experiment, the best fit from the steady-state coherence equations gives $\Gamma_{p l}^{-1}=20.4 \mu \mathrm{s}$ and $\Gamma_{t 0}^{-1}$ (ISC from $T_{1} \rightarrow S_{0}$ ) $=37.6 \mu \mathrm{s}$. Using Eq. (62), we obtain for the same values of $\gamma$ and $\tau_{0}, \Gamma_{p l}^{-1}=20.7$ $\mu \mathrm{s}$. Similarly, from a LADS experiment: for $\tau_{0}$ $=14.7 \mu \mathrm{s}$ and $\gamma=0.82, \Gamma_{p l}^{-1}=16.3 \mu \mathrm{s}$ and $\Gamma_{l 0}^{-1}=26.6 \mu \mathrm{s}$ from the steady-state coherence equations, and $\Gamma_{p l}^{-1}$ $=16.4 \mu \mathrm{s}$ from Eq. (62), thus ensuring the self-consis tency of the high power approximation. (Note that as mentioned in the text, if LADS and the EO experiments were done under identical conditions, then $\tau_{0}$ is exactly the same. ${ }^{89}$ ) The important conclusion is therefore that experimentally we can determine the rate of ISC especially if $\Gamma_{p l}$ and $\Gamma_{l 0}$ are much different. This is certainly true for the higher energy sites of pentacene in $p-$ terphenyl at $1.8 \mathrm{~K}$. Site $0_{3}$ gives a very large overshoot $\gamma=1.5$, and $\tau_{0} \simeq 6 \mu \mathrm{s} .{ }^{90}$ If in pentacene the ISC rate was $10^{8} \mathrm{~s}^{-1}$, as in many other aromatics, the overshoot depending on the power would be enormous and the decay during the pulse would be relatively very fast.

The interesting question is: What is the driving force for ISC? For direct $S_{1}$ to $T_{1}$ coupling (i. e., no other triplets involved), the ISC of $S_{1}$ to $T_{1}$ will be comparable to that of $T_{1} \rightarrow S_{0}$ if we assume that the electronic matrix element for the coupling and the density of states weighted Franck-Condon factors are similar, consider ing a Golden Rule type expression for the rates. The lifetime of the $T_{1}$ state is $33 \mu \mathrm{s}$ in the vapor and $70 \mu \mathrm{s}$ in solution and $38 \mu \mathrm{s}$ from our mixed crystal work (us ing the theoretical fit). The energy of $T_{1}$ is approximately half way between $S_{0}(|0\rangle)$ and $S_{1}(|p\rangle)$, see Table II. Since the energy gap between $T_{1}$ and $S_{1}$ exceeds the $4000 \mathrm{~cm}^{-1}$ range, $\mathrm{C}-\mathrm{H}$ stretching modes are presumably the dominant accepting vibrations and the FranckCondon weighted density of states should not be very dependent on which states are participating. Using the results of the energy gap law for ISC, one predicts an ISC rate on the order of $10^{4} \mathrm{~s}^{-1}$ for an energy gap of $\sim 8000 \mathrm{~cm}^{-1}$ between the excited singlet and $T_{1}$ of pentacene. Thus, on these grounds, we may conclude that $\Gamma_{p l}$ and $\Gamma_{l 0}$ are comparable. From our results on the $0_{1}$ site this is true, suggesting that the driving force is a direct ISC.

On the other hand, if $T_{2}$ is slightly below $|p\rangle$ in energy, then the driving force will be different. Internal conversion between $T_{2}$ and $T_{1}$ will be fast $\left(10^{10}-10^{14} \mathrm{~s}^{-1}\right)$ and this helps the irreversibility of the crossing. According to Gillispie and $\mathrm{Lim},{ }^{87}$ the ISC rate is related to $\Gamma_{T_{2} T_{1}}\left(T_{2}-T_{1}\right.$ rate of internal conversion) by the square of the ratio of the spin-orbit coupling matrix element to the energy difference between $|p\rangle$ and $T_{2}$. Thus for a rate of $10^{5} \mathrm{~s}^{-1}$ and $\Gamma_{T_{2} T_{1}} \simeq 10^{11} \mathrm{~s}^{-1}$, the $T_{2}$ state should be below $|p\rangle$ by $\simeq 1000 \mathrm{~cm}^{-1}$, knowing that the spin-orbital coupling matrix element is typically $1 \mathrm{~cm}^{-1}$ in these aromatics. This is the same reason which makes $\mathrm{Lim}^{87}$ believe that in pyrene there is no reason to assume that $T_{2}$ and $T_{3}$ must be above $S_{1}$ in order to explain the low rate of ISC. Whatever the driving force is, it is clear from our data that the rate of depleting $|p\rangle$ into $T$ must be conditioned by the observed slow ${ }^{91}$ light-on transient.

The above conclusions about the nature of $\{|l\rangle\}$ are supported by the different experiments performed. The Zeeman effect results in (average) shifting of the levels, and thus influences $\Gamma_{p l}$. The fact that $\tau_{0}$ is temperature independent in the range $1.8-4.2 \mathrm{~K}$ is also consistent, since a nearby triplet state (essentially in resonance) must be around in order to see temperature-enhanced ISC by $\sim 3 \mathrm{~cm}^{-1}$ phonon energies. The peak of the IRD decreases because the homogeneous width is increasing. The decrease in $\tau_{0}$ on going to site $0_{3}$ suggests that the $T_{2}$ state is becoming more involved. Since the energy gap between $|p\rangle$ and $T_{1}$ is very large, sensitivity of ISC to a site energy shift of $120 \mathrm{~cm}^{-1}$ is not expected. On the other hand, if $T_{2}$ is very close (1000 $\mathrm{cm}^{-1}$ or so) then such energy changes will influence the ISC rate. For the same value of $\Gamma_{T_{2} T_{1}}$, if $\tau_{0}$ becomes shorter by a factor fo $3\left(0_{1}\right.$ vs $0_{3}$ sites $)$, then $T_{2}$ shifts to only $580 \mathrm{~cm}^{-1}$ below $|p\rangle$. It is interesting to note that these sites, $0_{3}$, and $0_{4}$ have lifetimes very close to the gas phase value (see Table II) and are much different from the lifetime of $0_{1}$ and $0_{2}$ sites. This would be consistent with the "near" resonance role played by higher triplet states. Finally, all other observations are consistent including the fast vibrational relaxation in the solid which explains the absence of transients when the single mode of the laser was on resonance with the $267 \mathrm{~cm}^{-1}$ vibronic origin. The homogeneous width of this transition is larger than the 0,0 linewidth (i. e., the effective pumping rate is much less) and vibrational relaxation is fast enough to deplete population into $|p\rangle$.

\section{SUMMARY AND CONCLUSIONS}

In this paper we have attempted to address the question of what happens to large molecules, specifically pentacene, excited by narrow - and wide-band lasers focusing on the radiationless decay (optical $T_{1}$ ) and the phase relaxation (optical $T_{2}^{\prime}$ ) processes that the molecule undergoes. The following summary highlights some of the important points and observations.

(1) Optical $T_{2}$ has been measured using nutation and free induction decay techniques. For pentacene in a $p$ - 
terphenyl host at $1.8 \mathrm{~K}, T_{2}(44 \mathrm{~ns}) \simeq 2 T_{1}(23.5 \mathrm{~ns})$, suggesting that spontaneous emission to the ground state is causing the dephasing of the "two-level" system, in agreement with other results. ${ }^{49,52}$ Optical $T_{2}$, which has contributions from $T_{1}$ and $T_{2}^{\prime}$ processes changes as a function of temperature while $T_{1}$ does not. This was explained by the possible presence of quasilocalized or resonance modes, which have large amplitude at the guest impurity and are $\sim 17 \mathrm{~cm}^{-1}$ away from the origin. We also did not rule out other possibilities (like $T^{7}$ ).

(2) Optical $T_{1}$ is caused by radiative and nonradiative decay to the ground state while $T_{2}^{\prime}$ is caused by the difference in the scattering amplitudes between the ground and excited states [see Eq. (52)]. This matrix element difference, together with the phonon occupation number and density of states provide the temperature dependence and detail the origin of pure dephasing processes. In obtaining expressions for these dephasing rates we have shown that the origin of dephasing can be explained without requiring four levels in the crystal and that exchange between transitions will provide similar temperature dependence. We also present expres sions for cross sections of $T_{2}^{\prime}$ and $T_{1}$ processes.

(3) Experimentally, we have learned several things in doing these transients to obtain $T_{1}$ and $T_{2}$. First, when the crystal absorbs too much light (e.g. , singlet states) one has to be careful about radiation trapping that makes $T_{1}$ appear longer. Coherent emission, on the other hand, when reabsorbed does not seem to influence the $T_{2}$ measurement if one is confined to the lim. it of optically thin samples that obey Beer-Lambert's law. Second, it is very important to identify the effects of crystal inhomogeneous broadening and laser spatial transverse profile on the observed transients especially in the nutation experiments. Third, crystals with good optical quality are essential for observing the coherent transients in the forward direction of the laser. Finally, the laser pulse repetition rate could influence the observed decay characteristics for all the transients if it was high enough such that population in the $\{|l\rangle\}$ manifold had not returned completely to the ground state. To insure that each laser pulse found the system at equilibrium, the repetition rate was kept at $\leq 10 \mathrm{KHz}$ in our experiments where no effects on the transient decay were observed.

(4) The pentacene level structure was handled theoretically by dividing the problem into two time regimes: the coherent transient regime, where the time of observation is comparable to $T_{2}$ and the Rabi time, and the steady state coherence regime where the time of observation is much longer than $T_{2}$. In the latter regime we used the Wilcox-Lamb theory which enables one to use density matrix equations rather than a purely kinetic approach (where averaging over inhomogeneous broadenings, etc., is added phenomenologically). In this manner $\{|l\rangle\}$ was included in the equation of motion directly. For the transient coherence regime, where the off-diagonal elements of the density matrix have not decayed the transients were handled using the two-level system approach available in the literature and modified to suit the pentacene level structure.
(5) Because of $|l\rangle$, coherent transients of large molecules show unique and additional features (that do not exist in the two-level small molecule limit) which depend on whether the transient is observed following longtime preparation or using short pulses. Since optical free induction decay transients occur when the laser is switched off-resonance, one is monitoring the decay of the off-diagonal density matrix elements from some initial value [usually steady state, i.e., $\rho_{0 p}(\infty)$ ]. This can be done using Eq. (39) setting the time derivatives equal to zero. The result simply involves replacing $T_{1 p 0}$ in Eqs. (34)-(36) by $\left(2 \Gamma_{20}+\Gamma_{p l}\right) /\left\{\Gamma_{l 0}\left(\Gamma_{p 0}+\Gamma_{p l}\right)\right\}$. Thus, inclusion of the $\{|l\rangle\}$ manifold in the OFID expression does not af fect the transient decay at low powers. It does, how ever, increase the influence of the power broadening term upon the observed decay at high power, and alters the calculation of $\mu$.

(6) Optical $T_{2}$, in general, does not necessarily equal $2 T_{1}$, and thus calculation of $T_{1}$ from line shape analysis could be misleading. From Fig. 23, where we fit the observed high-resolution spectra (of low absorbance crystals) into Lorentzians or Gaussians, it is clear that one has to be careful about obtaining dephasing times from the analysis. For the 0,0 transitions of $0_{1}$ and $\mathrm{O}_{2}$ we could not obtain a good Gaussian fit which is "usually" indicative of inhomogeneous broadening. This is perhaps due to self-absorption and/or the incomplete randomness of impurity distribution in the host. We note that these lines must be inhomogeneously broadened since we know $T_{2}$ exactly. For the vibronic bands, the best fits we obtained (OD $\sim 0.015)$ are a Lorentzian for $0_{2}$ and a Gaussian for $0_{1}$. Taking a Lorentzian line shape as a criterion for homogeneous broadening we calculate a dephasing time of 5.4 ps. If vibrational relaxation is the dominant dephasing channel at these low temperatures (i. e. , $T_{2 v}=2 T_{1 v}$ ), then the vibrational relaxation time is $2.7 \mathrm{ps},{ }^{92}$ and the ratio of the homogeneous width of the 0,0 to $0, v\left(267 \mathrm{~cm}^{-1}\right)$ is $\sim 10^{-4}$.

(7) As far as the theoretical treatment goes, the detailed structure of the $\{|l\rangle\}$ manifold does not enter into the picture directly, unless we are interested in the details of intramolecular relaxation (internal conversion and intersystem crossing). We share the view of Lim et al. ${ }^{87}$ that higher triplets could be involved in these aromatics and we present some observations that are consistent with these ideas. The experiments show that the rate of intersystem crossing in pentacene is slow even though the crossing to the bottom of $T_{1}$ may proceed by fast vibrational relaxation or fast electronic $\left(T^{\prime} \rightarrow T_{1}^{\prime}\right)$ internal conversion.

(8) The yield for ISC based on the above results is about $2 \times 10^{-3}$, i. e., two molecules for every $1000|p\rangle$ molecules make it to the triplet state. Note that this number might confuse the reader because we mentioned before that $\gamma$ could be as large as 1.5 (e. g., for site 3 ). In other words, a very large fraction of the molecules is in the triplet. This is true because the pulse has been on for a long time, and thus molecules are constantly fed into the triplet. By triplet, of course, we mean the vibronic states with the spin substates $X, Y$, and $Z$. The reason we ignored being explicit with these spin states is because they do not add any new feature into our treatment since we assume that the total population in 
ABSORPTION LINESHAPES FOR PENTACENE/P-TERPHENYL AT $1.8^{\circ} \mathrm{K}$

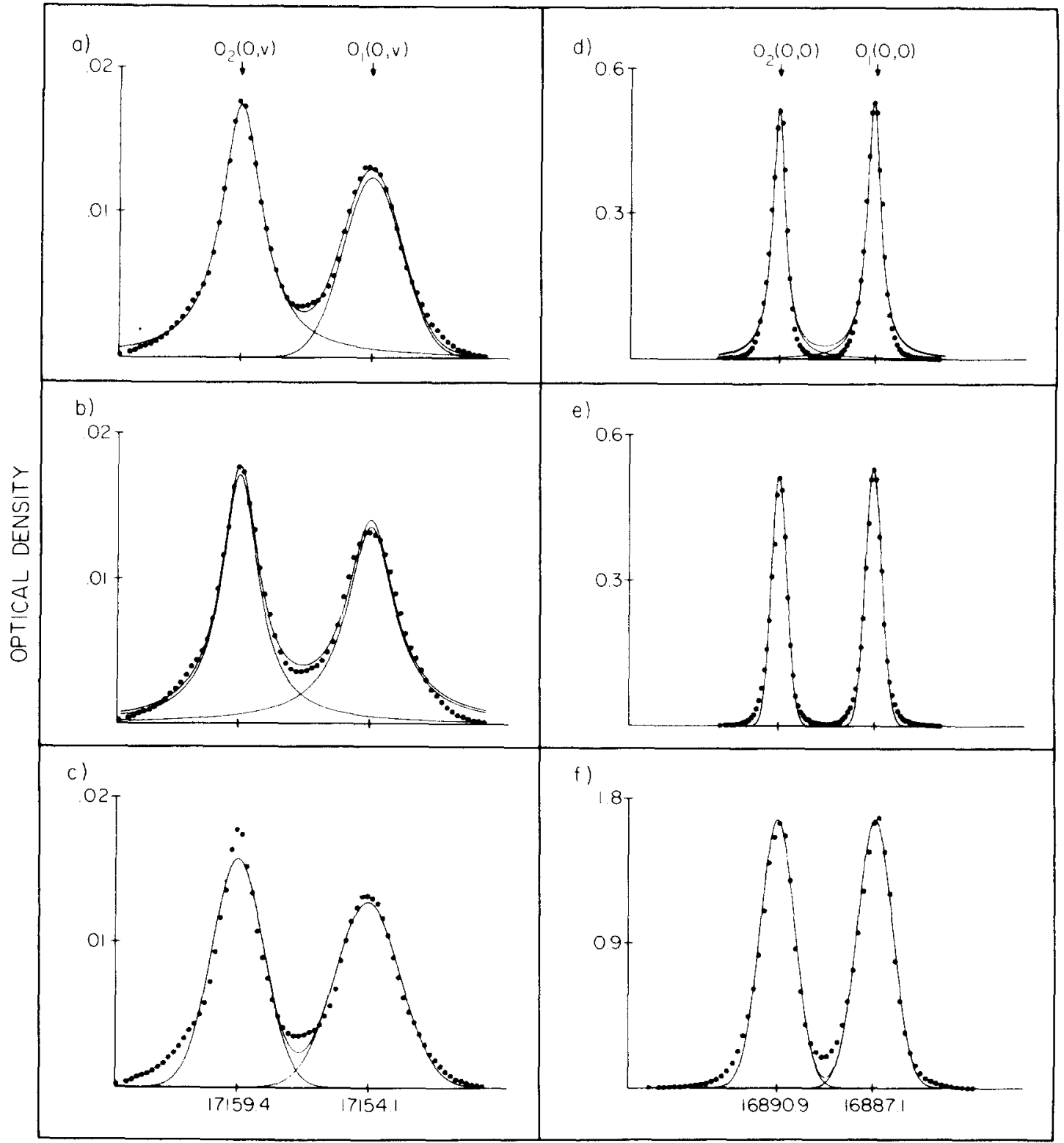

WAVENUMBERS

Fig. 23. Absorption line shapes for pentacene in p-terphenyl at $1.8 \mathrm{~K}$. Experimental data (solid circules) and line shape functions (solid lines) are shown for the vibronic lines (left) and origins (right) of sites $0_{1}$ and $0_{2}$. Attempts are made to fit each line to either a Lorentzian (L) or Gaussian (G) by a least squares curve fitting computer program. The program plots the best fit for each line and also the sum. The results are (FWHM linewidths in $\mathrm{cm}^{-1}$ appear in parentheses): (a) $0_{1}=\mathrm{G}(2.82), 0_{2}=\mathrm{L}(1.99)$; (b) $0_{1}=\mathrm{L}(2.36)$, $0_{2}=\mathrm{L}(1.81) ;$ (c) $0_{1}=\mathrm{G}(3.12), 0_{2}=\mathrm{G}(2.48) ;$ (d) $0_{1}=\mathrm{L}(0.66), 0_{2}=\mathrm{L}(0.61) ;$ (e) $0_{1}=\mathrm{G}(0.79), 0_{2}=\mathrm{G}(0.74) ;(\mathrm{f}) 0_{1}=\mathrm{G}(1.69), 0_{2}=\mathrm{G}(1.65)$. Spectrs $(\mathrm{a})-(\mathrm{e})$ were obtained with a low concentration $\left(\approx 1 \times 10^{-6} \mathrm{~m} / \mathrm{m}\right)$ crystal and spectrum (f) was taken with a higher concentration $\left(\approx 5 \times 10^{-6} \mathrm{~m} / \mathrm{m}\right)$ crystal.

$|p\rangle,|0\rangle$, and $|l\rangle$ is always constant. Our $\rho$ does not in a direct way care about the fine structure of $l$ and takes the following form:

$$
\rho=\left(\begin{array}{lllll}
\rho_{00} & \rho_{0 p} & & & \\
\rho_{p 0} & \rho_{p p} & & & \\
& & \rho_{l_{1} l_{1}} & & \\
& & & \rho_{l_{2} l_{2}} & \\
& & & & \rho_{l_{x} l_{x}}
\end{array}\right)
$$

and

$$
\operatorname{Tr} \rho=1 \text {. }
$$

(9) In the pentacene system (extinction coefficient $\sim 10^{4}$ ), the on- and off-resonance scattering experiments reveal a long transient decay and also the importance of self-absorption which is responsible for the inverted transient pattern in Figs. 20 and 22 . These onand off-resonance scattering experiments are useful in that they are sensitive in part to ground state population. Excellent fit between theory and experiments in pentacene was found. 
FOURIER TRANSFORMS OF LASER PULSES

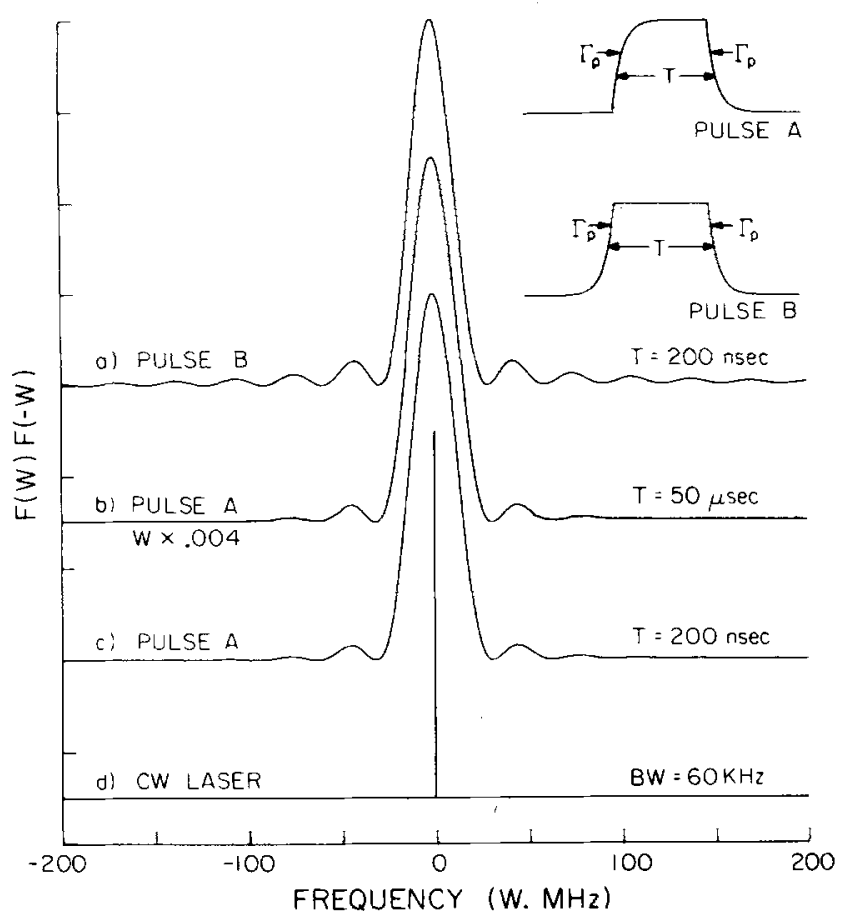

FIG. 24. Depicted here are Fourier transforms of laser pulses. Pulse $A$ and $B$ have identical widths $T$, and rise and fall times, $\Gamma_{p}^{-1}$ of $10 \mathrm{~ns}$. Spectra (a) and (c) correspond to $200 \mathrm{~ns}$ wide laser pulses and their bandwidths (FWHM) are 26.1 and $27.5 \mathrm{MHz}$, respectively. Spectrum (b) corresponds to a $50 \mu \mathrm{s}$ wide $A$ pulse and its bandwidth is $111.3 \mathrm{kHz}$. Shown at the bottom [spectrum (d)] is the effective bandwidth $(60 \mathrm{kHz}$, see Ref. 93) of the single-mode cw dye laser on the time scale of the slow transients observed in pentacene.

(10) Finally, narrow -band excitation of molecules with narrow -band $\mathrm{cw}$ lasers demands knowledge of the exact pulse (EO and LADS) shape and width in order to determine the effective bandwidth and the coherence properties of the source. For the pentacene studies we can vary this effective width from essentially the residual width of $\mathrm{cw}$ source to values exceeding the homogeneous line width of the transition (7.1 MHz), as shown in Fig. 24.

\section{ACKNOWLEDGMENTS}

Acknowledgment is made to the Donors of the $\mathrm{Pe}$ troleum Research Fund, administered by ACS, for partial support of this work. This work was also supported by a grant from the National Science Foundation. We would like to acknowledge the contribution of and discussion with several people during the course of this work. W. Lambert has helped us in obtaining the results on the line shape analysis and some of the LADS experiments. $K$. Jones has contributed to Sec. VF and discussions with him throughout the work were productive. A. Nichols has provided us with the solution of the integral in Appendix II. Finally, we would like to thank Dr. N. J. Bridge and Dr. A. Lami and Dr. P. Grigolini for communicating unpublished results and Professor J. Jortner for stimulating discussions.

\section{APPENDIX A: FOURIER TRANSFORMS OF THE LASER PULSES USED IN THIS WORK}

The effective bandwidth of the $\mathrm{cw}$ single-mode laser varies between $60 \mathrm{kHz}$ and $6 \mathrm{MHz}$ depending upon the time scale of the experiment. When we utilize the EO and LADS techniques to produce pulses from this laser, frequency broadening will occur as the pulse width decreases. To quantify this effect we calculated Fourier transforms for various pulse shapes and widths (see Fig. 24) in order to determine a lower limit to the frequency bandwidth of the single-mode dye laser in our experiments.

If $f(t)$ represents the functional form of the laser pulse in time, then its Fourier transform will be defined as:

$$
F(\omega)=C_{N} \int_{-\infty}^{\infty} f(t) \exp (-i \omega t) d t,
$$

where $C_{N}$ is a normalization factor such that the value of the transform at the center frequency (here $\omega=0$ ) is 1. For the pulses shown in Fig. 24, which have identical widths $T$, and rise and fall times $\Gamma_{p}^{-1}$, the transforms are:

$$
\begin{aligned}
F_{A}(\omega)= & C_{N}\left\{\frac{2 \sin \omega T / 2}{\omega}+\left(\frac{\Gamma_{p}-i \omega}{\Gamma_{p}^{2}+\omega^{2}}\right)\right. \\
& \times[\exp (-i \omega T / 2)-\exp (i \omega T / 2)]\}
\end{aligned}
$$

and

$$
\begin{aligned}
F_{B}(\omega)= & C_{N}^{\prime}\left\{\frac{\sin \omega T / 2}{\omega}+\frac{1}{\Gamma_{p}^{2}+\omega^{2}}\right. \\
& \left.\times\left[\Gamma_{p} \cos \frac{\omega T}{2}+\omega \sin \frac{\omega T}{2}\right]\right\} .
\end{aligned}
$$

To obtain $F_{A}(\omega)$ as shown in Eq. (A2), a term containing $\exp \left(-\Gamma_{p} T / 2\right)$ was ignored. This is valid for our experiments where $T \gg \Gamma_{p}^{-1}$ such that $\exp \left(-\Gamma_{p} T / 2\right) \approx 0$. Since $F_{A}(\omega)$ is complex, we will calculate the energy spectra of the pulses which are always real functions. The energy spectrum is related to the Fourier transform in the following way:

$$
S(\omega)=F(\omega) F(-\omega) .
$$

For pulse $A$,

$$
S_{A}(\omega)=4 C_{N}^{2} \frac{\sin ^{2} \omega T / 2}{\omega^{2}} \frac{\Gamma_{D}^{2}}{\Gamma_{D}^{2}+\omega^{2}}
$$

and for pulse $B$,

$$
S_{B}(\omega)=F_{B}^{2}(\omega),
$$

since $F_{B}(\omega)$ is real. Equations (A5) and (A6) are plotted vs $\omega$ in Fig. 24 for a pulse width of $200 \mathrm{~ns}$ which is the width used in the OFID and nutation experiments. Neglecting the small sidebands, the frequency bandwidths (FWHM) are 27. 5 and 26. $1 \mathrm{MHz}$ for $A$ and $B$ pulses, respectively. The energy spectrum of pulse $A$ is slightly broader than that of pulse $B$. This occurs because the area under pulse $A$ is less than that of $B$. Continuing along these lines, the energy spectrum of a step-function pulse (infinitely fast rise and fall time) of the same width as $A$ and $B$ is even broader $(F W H M=27.8 \mathrm{MHz}$ ).

In our experiments, pulse $A$ accurately describes the pulses we observe using both the EO and LADS tech- 
niques. Therefore, we used this pulse to determine the bandwidth of the long laser pulses used in IRD experiments. Shown in Fig. 24 is the energy spectrum [Eq. (A5) vs $\omega$ ] for a $50 \mu$ s $A$ pulse. Here, the frequency scale has been reduced $(\omega \times 0.004)$ indicating that the bandwidth for this pulse is much narrower (FWHM $=111.3 \mathrm{kHz}$ ).

Now, since the experiments are performed with a train of pulses rather than just one the effects of repetition rate on the form of the transforms must be considered. If the laser pulse is repeated ad infinitum at regular intervals $T_{i}$, where $T_{i}$ is the pulse separation, then all of the transform is removed except for delta function samples at $\omega= \pm n\left(2 \pi / T_{i}\right)$ where $n$ is an integer. However, the envelope of the transform does not change. Most of the experiments on pentacene were performed at pulse repetition rates of $10 \mathrm{kHz}$. Therefore, the energy spectra for pulse trains are identical to those shown in Fig. 24 (i. e., they have the same overall width) except that they have delta function samples separated by approximately $63 \mathrm{kHz}$.

Finally, to illustrate the broadening effects introduced by pulsing a $\mathrm{cw}$ laser, shown at the bottom of Fig. 24 is the effective bandwidth of the single-mode dye laser on the time scale of the slow transients observed in pentacene. Ezekiel and $\mathrm{Wu}$ have shown ${ }^{93}$ that the line width is $60 \mathrm{kHz}$ ( $\mu \mathrm{s}$ time scale) and determined primarily by residual FM jitter.

The important points of this section are: (1) for short (200 ns) pulses, the bandwidth of the single-mode laser is temporally limited and almost $\mathbf{5 0 0}$ times larger than the effective $\mathrm{cw}$ bandwidth and (2) for long $(50 \mu \mathrm{s})$ pulses the laser bandwidth is determined almost entirely by residual FM jitter.

\section{APPENDIX B: APPROXIMATE SOLUTIONS FOR INTEGRALS IMPORTANT FOR INHOMOGENEOUS LINEWIDTH AVERAGING}

The solution of the integral written here in general form as:

$$
I=\frac{1}{\sqrt{\pi} \beta} \int_{-\infty}^{\infty} \exp \left(\frac{-\alpha}{\gamma^{2}+\Delta^{2}}-\frac{\Delta^{2}}{\beta^{2}}\right) d \Delta
$$

is required to determine both $r_{2}(t)$ [Eq. (15)] and $\rho_{p p}(t)$ (in the high power limit of the steady-state coherence regime) averaged over the inhomogeneous linewidth. Since, to our knowledge, there is no analytic solution for this integral, it is usually approximated as:

$$
I=\frac{1}{\sqrt{\pi} \beta} \int_{-\infty}^{\infty} \exp \left(\frac{-\alpha}{\Delta^{2}}-\frac{\Delta^{2}}{\beta^{2}}\right) d \Delta
$$

which is analytic. To show that this approximation is valid in general for $\gamma^{2} \ll \beta^{2}$ the integral may be evaluated by an asymptotic expansion of the exponential in a Taylor series about the maxima of the argument.

With the argument of the exponential given by:

$$
f(\Delta)=\frac{-\alpha}{\gamma^{2}+\Delta^{2}}-\frac{\Delta^{2}}{\beta^{2}}
$$

taking successive derivatives reveals that maxima in $f(\Delta)$ occur at $\Delta= \pm\left(\sqrt{\alpha} \beta-\gamma^{2}\right)^{1 / 2}$ if $\alpha \beta^{2}>\gamma^{4}$, and at $\Delta=0$ if $\alpha \beta^{2}<\gamma^{4}$. Expanding $f(\Delta)$ in a Taylor series about $\Delta$ $=\left( \pm \sqrt{\alpha} \beta-\gamma^{2}\right)^{1 / 2}$ and keeping only the first term in $\Delta$ one obtains:

$f(\Delta)=-\left(\frac{2 \sqrt{\alpha}}{\beta}-\frac{\gamma^{2}}{\beta^{2}}\right)-4\left(\frac{1}{\beta^{2}}-\frac{\gamma^{2}}{\alpha^{1 / 2} \beta^{3}}\right)\left(\Delta-\left( \pm \sqrt{\alpha} \beta-\gamma^{2}\right)^{1 / 2}\right)^{2}$.

Therefore, the integral $I$ may be expressed as:

$$
\begin{aligned}
I & \approx \frac{1}{\sqrt{\pi} \beta} \sum_{\substack{\text { over } \\
\pm \text { roots }}} \exp \{f(\Delta)\} d \Delta \\
& =\frac{2}{\sqrt{\pi} \beta} \exp \left[-\left(\frac{2 \sqrt{\alpha}}{\beta}-\frac{\gamma^{2}}{\beta^{2}}\right)\right] \frac{\sqrt{\pi}}{2\left(1 / \beta^{2}-\gamma^{2} / \alpha^{1 / 2} \beta^{3}\right)^{1 / 2}} \\
& =\frac{\exp \left[-\left(2 \sqrt{\alpha} / \beta-\gamma^{2} / \beta^{2}\right)\right]}{\left(1-\gamma^{2} / \alpha^{1 / 2} \beta\right)^{1 / 2}} .
\end{aligned}
$$

Using the same techniques, the result for the integral with $f$ expanded about $\Delta=0$ is:

$$
I \approx \frac{\exp \left(-\alpha / \gamma^{2}\right)}{\left(1-\alpha \beta^{2} / \gamma^{4}\right)^{1 / 2}}
$$

For our purposes, the useful result will be Eq. (B6). This can be shown by substituting for $\alpha, \gamma$, and $\beta$ the appropriate variables from Eq. (15) for $r_{2}(t)$ and Eq. (60) for $\rho_{p p}(t)$.

First, from Eq. (15), $\alpha=\chi^{2} t / 2 T, \gamma=\chi$ and $\beta=\delta \omega_{1}$. From experiment, $\chi=0.23 \mathrm{GHz}$ (see p. 46), $T=\left(T_{1 p 0}^{-1}\right.$ $\left.-T_{2}^{-1}\right)^{-1}=56.3$ ns and $\delta \omega_{I}=24 \mathrm{GHz}$ (see Fig. 15). Thus, $\alpha \beta^{2} \gg \gamma^{4}$ when $t \gg 0.2 \mathrm{~ns}$. Therefore, Eq. (B6) is valid for describing all but the very short time behavior of $\left\langle r_{2}(t)\right\rangle$. Furthermore, considering the above values of the parameters, Eq. (B6) reduces to the form shown in Eq. (18) for $\left\langle r_{2}(t)\right\rangle$ which was obtained using the approximation shown in (B2). Now, from the appropriate integral for $\rho_{p \rho}(t)$ in Eq. (60), $\alpha=\chi^{2} \Gamma t, \gamma=\Gamma$, and $\beta$ $=\delta \omega_{P B}$. With these parameters $R_{p_{\text {efI }}}=\left(\chi / \delta \omega_{P B}\right) \sqrt{\Gamma / t}$ which is identical to the previous result.

${ }^{1}$ G. B. Kistiakowsky and C. S. Parmenter, J. Chem. Phys. 42, $2942(1965)$; E. M. Anderson and G. B. Kistiakowsky, ibia. 48, 4787 (1968); C. S. Parmenter and A. H. White, ibid. 50, 1631 (1969).

${ }^{2}$ A. E. Douglas, J. Chem. Phys. 45, 1007 (1967).

${ }^{3}$ For reviews on the subject see: G. W. Robinson, in Excited States, edited by E. C. Lim (Academic, New York, 1974), Vol. I, p. 1; S. A. Rice, in Excited States, edited by E. C. Lim (Academic, New York, 1975), Vol. 2, p. 111; J. Jortner and S. Mukamel, in The World of Quantum Chemistry, edited by R. Daudel and B. Pullman (Reidel, Boston, 1974); K. F. Freed, Topics Appl. Phys. 15, 23 (1976).

${ }^{4} \mathrm{G}$. W. Robinson and R. P. Frosch, J. Chem. Phys. 37, 1962 (1962); 38, 1187 (1963).

${ }^{5} \mathrm{M}$. Bixon and J. Jortner, J. Chem. Phys. 50, 3284, 4061 (1969).

${ }^{6}$ U. Fano, Phys. Rev. 124, 1866 (1961).

${ }^{7}$ K. F. Freed, J. Chem. Phys. 52, 1345 (1970).

${ }^{8}$ W. Rhodes, J. Chem. Phys. 50, 2885 (1969); W. Rhodes, R. B. Henry, and M. Kasha, Proc. Natl. Acad. Sci. USA 63, 31 (1969); W. Rhodes, Chem. Phys. Lett. 11, 179 (1971); W. Rhodes, Chem. Phys. 4, 259 (1974); 22, 95 (1977).

${ }^{9} \mathrm{C}$. A. Langhoff and G. W. Robinson, Mol. Phys. 26, 249 (1973); 29, 613 (1975); C. A. Langhoff, Chem. Phys. 20 , 357 (1977).

${ }^{10} \mathrm{~W}$. Weisskopf and E. Wigner, Z. Phys. 63, $54(1930)$.

${ }^{11}$ A. H. Zewail, T. E. Orlowski, and K. E. Jones, Proc. Natl. Acad. Sci. USA 74, 1310 (1977); see also Ref. 12. 
${ }^{12}$ A. H. Zewail, T. E. Orlowski, and D. R. Dawson, Bull. Am. Phys. Soc. 21, 1283 (1976); A. H. Zewail, Proc. SPIE 82, $26(1976)$; A. H. Zewail, D. E. Godar, K. E. Jones, T. E. Orlowski, R. R. Shah, and A. Nichols, in Advances in Laser Spectroscopy I, edited by A. H. Zewail (SPIE, 1977), Vol. 113, p. 42.

${ }^{13}$ M. Kasha, Chem. Rev. 41, 401 (1947); H. B. Klevens and J. R. Platt, J. Chem. Phys. 17, 470 (1949); R. Pariser, ibid. 24, 250 (1956); D. R. Kearns, ibid. 36, $1608(1962)$; T. E. Peacock and P. T. Wilkinson, Proc. Phys. Soc. 83, 525 (1964); G. G. Hall, Proc. R. Soc. London Ser. A 213, 113 (1952).

${ }^{14}$ N. A. Kurnit, I. D. Abella, and S. R. Hartmann, Phys. Rev. Lett. 13, $567(1964)$; G. B. Hocker and C. L. Tang, ibid. 21, 591 (1968); N. Takeuchi and A. Szabo, Phys. Lett. A 50, 361 (1974); R. G. Brewer and R. L. Shoemaker, Phys. Rev. A 6, 2001 (1972); R. G. Brewer and A. Genack, Phys. Rev. Lett. 36, 959 (1976).

${ }^{15}$ A. H. Zewail, T. E. Orlowski, and D. R. Dawson, Chem. Phys. Lett. 44, 379 (1976); A. H. Zewail, T. E. Orlowski, K. E. Jones, and D. E. Godar, ibid. 48, 256 (1977); A. H. Zewail, D. E. Godar, K. E. Jones, T. E. Orlowski, R. R. Shah, and A. Nichols, in Advances in Laser Spectroscopy I, edited by A. H. Zewail (SPIE, Bellingham, Washington, 1977), Vol. 113, p. 42.

${ }^{16}$ G. B. Hocker and C. L. Tang, Phys. Rev. 184, 356 (1969); R. G. Brewer and R. L. Shoemaker, Phys. Rev. Lett. 27, 631 (1971); C. L. Tang and B. D. Silverman, Physics of Quantum Electronics, edited by Kelley, Lax, and Tannewald (McGraw-Hill, New York, 1966).

${ }^{17}$ R. G. Brewer and R. L. Shoemaker, Phys, Rev. A 6, 2001 (1972); F. A. Hopf, R. F. Shea, and M. O. Scully, ibid. 7, 2105 (1973).

${ }^{18}$ T. E. Orlowski, K. E. Jones, and A. H. Zewail, Chem. Phys. Lett. 54, 197 (1978).

${ }^{19}$ W, R. Harshbarger and M. B. Robin, Acc. Chem. Res. 6(2) 329 (1973).

${ }^{20}$ A. Yariv, Proc. IEEE 52, 719 (1964).

${ }^{21}$ J. E. Kiefer, T. A. Nussmeier, and F. E. Goodwin, IEEE J. Quant. Electron. QE-8, 173 (1972).

${ }^{22}$ J. L. Hall, Atomic Physics 3, edited by S. J. Smith, G. K. Walters, and L. H. Volsky (Plenum, New York, 1973), p. 615.

${ }^{23}$ R. L. Shoemaker and E. W. Van Stryland, J. Chem. Phys. 64,1733 (1976). See also p. 1739 of this reference, and Ref. 94.

${ }^{24}$ R. G. Brewer and A. Genack, Phys, Rev. Lett. 36, 959 (1976).

${ }^{25} \mathrm{~A}$. H. Zewail and T. E. Orlowski, Chem. Phys. Lett. 45, 399 (1977); A. H. Zewail, D. E. Godar, K. E. Jones, T. E. Orlowski, R. R. Shah, and A. Nichols, in Advances in Laser Spectroscopy I, edited by A. H. Zewail (SPIE, Bellingham, Washington, 1977), Vol. 113, p. 42.

${ }^{26}$ A. Genack, R. M. Mcfarlane, and R. G. Brewer, Phys. Rev. Lett. 37, 1078 (1976).

${ }^{27}$ A. H. Zewail, T. E. Orlowski, R. R. Shah, and K. E. Jones, Chem. Phys, Lett. 49, 520 (1977).

${ }^{28} \mathrm{R}$. Adler, IEEE Spectrum 4, 42 (1967).

${ }^{29}$ G. Beck, Rev. Sci. Instrum. 47, $537(1976)$.

${ }^{30}$ S. W. Provencher, J. Chem. Phys. 64, 2772 (1976).

${ }^{31}$ D. W. Marquardt, J. Soc. Industrial Appl. Math. 11, 431 (1963).

${ }^{32}$ P. C. Haarhof, Mol. Phys. 7, 101 (1962).

${ }^{33}$ K. F. Freed, Topics Current Chem. 31, 105 (1972); W. M. Gelbart, D. F. Heller, and M. L. Elert, Chem. Phys. 7, 116 (1975); J. M. Delory and C. Tric, ibid. 3, 54 (1974).

${ }^{34}$ This kind of coherence in the excited state should not be confused with the coherence created between the excited state relative to the ground state by the laser field.

${ }^{35}$ See, e.g. , M. Sargent, M. O. Scully, and W. E. Lamb, Jr., Laser Physics (Addison-Wesley, Reading, MA, 1974); L. Allen and J. H. Eberly, Optical Resonance and Two-Level
Atoms (Wiley-Interscience, New York, 1975).

${ }^{36}$ P. Grigolini and A. Lami, Chem. Phys. Lett. 55, 152 (1978); P. Grigolini and A. Lami, Chem. Phys. 30, 61 (1978).

${ }^{37}$ R. P. Feymman, F. Vernon, and R. W. Hellwarth, J. Appl. Phys. 28, 49 (1957).

${ }^{38}$ It is important to note that in the NMR Ianguage on speaks of $T_{1}$ only when both levels are decaying by the same rate. In general, if the two levels have different rates, then the system appears statistically open and one must consider the time evolution of the total population as well as the other usual terms $\left(\rho_{p p}-\rho_{00}, \rho_{0 p}\right.$, and $\left.\rho_{p 0}\right)$. Under these circumstances one may group the decay rate terms that belong to the $\left(\rho_{p D}-\rho_{00}\right)$ expression and, perhaps, define these terms as the $T_{1}^{-1}$ rate constant [see Eq. (11)], keeping in mind that the definition of $T_{1}$ in the usual Bloch equations demands that the excited and ground state $T_{1}$ 's are approximately the same. Finally, one can see that the level structure we are considering for pentacene [described by Eq. (10)] represents a system where the ground state is not decaying and the population dynamics in the two levels is determined only through $T_{1 p 0}$. Note that from (10), $\dot{\rho}_{00}+\dot{\rho}_{p p}=0$ at all times, when $\chi=0$.

${ }^{39} \mathrm{~A}$. Schenzle and R. G. Brewer, Phys. Rev. A 14, 1756 (1976).

${ }^{40}$ T. E. Orlowski, K, E. Jones, and A. H. Zewail, Chem. Fhys. Lett, 50, 45 (1977).

${ }^{41}$ K. E. Jones, A. H. Nichols, and A. H. Zewail, J. Chem. Phys. 69, 3350 (1978).

${ }^{42}$ L. R. Wilcox and W. E. Lamb, Jr., Phys. Rev. 119, 1915 (1960); W. E. Lamb, Jr. and T. M. Sanders, Jr., ibid. 119, 1901 (1960).

${ }^{43}$ J. R. Ackerhalt and B. W. Shore, Phys. Rev. A 16, 277 (1977); J. R. Ackerhalt and J. H. Eberly, ibid. 14, 1705 (1976); B. L. Beers and L. Armstrong, Jr., ibid. 12, 2447 (1975).

${ }^{44}$ A. P. Marchetti, W. C. McColgin, and J. H. Eberly, Phys. Rev, Lett. 35, 387 (1975).

${ }^{45}$ N. J. Bridge, private communication.

${ }^{46}$ J. H. Meyling and D. A. Wiersma, Chem. Phys. Lett. 20, 383 (1973).

${ }^{47}$ G. J. Small, J. Chem. Phys. 52, $656(1970)$.

${ }^{48}$ R. M. Hochstrasser and P. N. Prasad, J. Chem. Phys. 56, 2814 (1972).

${ }^{49}$ T. J. Aartsma, J. Morsink, and D. A. Wiersma, Chem. Phys. Lett. 47, 425 (1977); 42, 520 (1976); 49, 34 (1977).

${ }^{50}$ N. J. Kruse and G. J. Small, J. Chem. Phys. 56, 2985 (1972).

${ }^{51}$ See Ref. 25 (Advances in Laser Spectroscopy I), and Ref. 18. ${ }^{52}$ (a) D. A. Wiersma, in The Proceeding of the Eighth Molecular Crystal Symposium, Santa Barbara, CA (1977); (b) H. de Vries, P. de Bree, and D. A. Wiersma, Chem. Phys. Lett. 52, 399 (1977); (c) H. de Vries, D. A. Wiersma, Abstract C2, Conference on Dynamical Processes in the Excited States of Ions and Molecules in Solids, Athens, GA (1978).

${ }^{53} \mathrm{~A}$. Matsuzaki and S. Nagakura, Chem. Phys. Lett. 37, 204 (1976); H. G. Kuttner, H. Selzle, and E. Schlag ibid. 48, 207 (1977).

${ }^{54}$ P. W. Atkins and P. R. Stannard, Chem. Phys. Lett. 47, 113 (1977).

${ }^{55} \mathrm{M}$. A. EI-Sayed, MTP International Reviews of Science Spectroscopy, edited by A. D. Buckingham and D. A. Ramsey (Butterworths, London, 1972), p. 119.

${ }^{56}$ K. F. Freed, J. Chem. Phys. 64, 1604 (1976).

${ }^{57}$ R. H. Clarke and H. A. Frank, J. Chem. Phys. 65, 39 (1976); R. H. Clarke and J. M. Hayes, Chem. Phys. Lett. 27, 556 (1974).

${ }^{58}$ T. Azumi, C. M. O'Donnell, and S. P. McGlynn, J. Chem. Phys. 45, 2735 (1966).

${ }^{59}$ R. H. Clarke, R. E. Connors, and J. Keegan, J. Chem. Phys. 66, 358 (1977).

${ }^{60}$ M. A. El-Sayed and R. Leyerle, J. Chem. Phys. 62, 1579 
(1975).

${ }^{61}$ (a) P. F. Williams, D. L. Rousseau, and S. H. Dworetsky, Phys. Rev. Lett. 32, $196(1974)$; (b) H. Gibbs and T. Venkatesan Opt. Commun. 17, 87 (1976).

${ }^{62}$ J. M. Friedman and R. M. Hochstrasser, Chem. Phys. 6, 155 (1974); J. O. Berg, C. A. Langhoff, and G. W. Robinson, Chem. Phys. Lett. 29, 305 (1974); S. Mukamel and J. Jortner, J. Chem. Phys, 62, 3609 (1975); S. Mukamel, A. Ben-Reuven, and J. Jortner, Phys. Rev. A 12, 947 (1975); R. M. Hochstrasser and F. Novak, Chem. Phys. Lett. 48, 1 (1977); J. Carlsten and M. G. Raymer, in Laser Spectroscopy, edited by J. Hall and J. Carlsten (Springer Series in Optic Sciences, Springer-Verlag, New York, 1977), Vol. 7; F. Novak, J. Friedman, and R. M. Hochstrasser, in Laser and Coherence Spectroscopy, edited by J. Steinfeld (Plenum, New York, 1978).

${ }^{63} \mathrm{~A} . \mathrm{H}$. Zewail, in Laser Spectroscopy, edited by J. Hall and J. Carlsten (Springer-Verlag, New York, 1977), Vol. 7, p. 268.

${ }^{64}$ The background scattered light off-resonance is almost a factor of 2 larger (at the end of the laser pulse) than the corresponding level on-resonance. This is not shown in Fig. 20 where data have been normalized by keeping the photomultiplier current the same.

${ }^{65}$ D. J. Diestler, Mol. Phys. 32, 1091 (1976).

${ }^{66}$ S. F. Fischer and A. Laubereau, Chem. Phys. Lett. 35, 6 (1975).

${ }^{67}$ D. Oxtoby and S. A. Rice, Chem. Phys. Lett. 42, 1 (1976).

${ }^{68} \mathrm{P}$. A. Madden and R. M. Lynden-Bell, Chem. Phys. Lett. 38, 163 (1976).

${ }^{69} \mathrm{D}$. Oxtoby, in Advances in Chemical Physics, edited by I. Prigogine and S. A. Rice (to be published)

${ }^{70}$ C. B. Harris, J. Chem. Phys. 67, 5607 (1978); also in Advances in Laser Chemistry, edited by A. H. Zewail (Springer Series in Chemical Physics, Springer-Verlag, New York, 1978). The last article summarizes some new findings.

${ }^{71}$ P. W. Anderson, J. Phys. Soc. Jpn. 9, 316 (1954); R. Kubo and K. lomita, ibid. 9, 888 (1954).

${ }^{72} \mathrm{~K}$. E. Jones and A. H. Zewail, in Advances in Laser Chemistry, edited by A. H. Zewail (Springer Series in Chemical Physics, Springer-Verlag, New York, 1978), p. 196.

${ }^{73}$ A. H. Zewail, K. E. Jones, and T. E. Orlowski, Spectrose. Lett. 10, 115 (1977); the proof of the master equation in this paper can be found in Ref. 72 .

${ }^{74}$ R. F. Snider, J. Chem. Phys. 32, 1052 (1960).

${ }^{75}$ W. Liu and R. A. Marcus, J. Chem. Phys. 63, 272 (1975).

${ }^{76} \mathrm{R}$. Zwanzig, in Lectures in Theoretical Physics, edited by W. E. Brittin (Interscience, New York, 1961), Vol. III, p. 106.

${ }^{77}$ U. Fano, Phys. Rev. 131, 259 (1973).

${ }^{78}$ A. Ben-Reuven, in Advances in Chemical Physics, edited by I. Prigogine and S. A. Rice (Wiley, New York, 1975), Vol. 23, p. 235.

${ }^{79} \mathrm{~K}$. E. Jones, A. H. Zewail, and D. Diestler, in Advances in Laser Chemistry, edited by A. H. Zewail (Springer Series in Chemical Physics, Springer-Verlag, New York, 1978), and references therein.

${ }^{80}$ D. E. McCumber, J. Math. Phys. 5, 221 (1964); D. E. McCumber and M. D. Sturge, J. Appl. Phys. 34, 1682 (1963); B. DiBartolo, Optical Interactions in Solids (Wiley, New York, 1968).

${ }^{81}$ (a) G. J. Small, J. Chem. Phys. 54, 3300 (1971); 58, 2015 (1973); (b) Note that using these phonon modes one can also obtain the familiar $T^{4}$ terms for the shift as well as the other forms of the high temperatures regime; (c) C. B. Harris, Chem. Phys. Lett. 52, 5 (1977).

${ }^{82}$ A. H. Zewail, J. Chem. Phys. (to be published).

${ }^{83}$ We should mention that when we quote temperatures in this paper we mean the temperature measured by the sensor very near the crystai. We do not know the actual temperature "inside" the crystal.

${ }^{84}$ In these fits we plot the intensity which is an exponential function of $1 / T_{2}$ which in turn changes with temperature according to the $T^{\eta}$ or the $e^{-\Delta / k T}$ dependence. We have found that these two fits are almost indistinguishable. The difference is largest between 4.2 and $6 \mathrm{~K}$ where we have no data. Note that for the $e^{-\Delta / k T}$ function the signal intensity is an exponential of an exponential.

${ }^{85}$ I. D. Abella, N. Kurnit, and S. R. Hartmann, Phys. Rev. 141, 391 (1966).

${ }^{36} \mathrm{At}$ low temperatures slow vibrational relaxation in the excited state of benzene in hydrogen was found by P. Frosch (G. W. Robinson, private communication). The slow relaxation implies that the benzene mode is not able to dump that high energy into the "inert" matrix.

${ }^{87}$ G. Gillispie and E. C. Lim, J. Chem. Phys. 65, 2022 (1976); E. C. Lim (private communication of unpublished work).

${ }^{88} \mathrm{~J}$. B. Birks, Photophysics of Aromatic Molecules (WileyInterscience, New York, 1970); E. Schlag, S. Schneider, and S. F. Fischer, Ann. Rev. Phys. Chem. 22, 465 (1971).

${ }^{89}$ Note that in an IRD experiment, we eliminate the dc background and amplify the ac transient signal which may be $10-30 \%$ of the dc level. For LADS transients we observe both the de and the ac components. Thus at very low powers, IRD can give much more accurate $\tau_{0}$ 's than LADS.

${ }^{90} \mathrm{~W}$. Lambert and A. Zewail, unpublished work.

${ }^{91}$ It is for the same reason that we exclude the possiblity that the observed slow decay is due to ground state vibrational relaxation. Because if so, the molecules will leave $|p\rangle$ at $(24)^{-1} \mathrm{~ns}^{-1}$ rate (spontaneous emission to ground states) and the overshoot will be relatively large and the light-on transient will decay rapidly.

${ }^{92}$ The early measurement of Marchetti et al. (Ref. 44) indicated that vibrational relaxation is much slower $\left(\sim 2 \times 10^{-10} \mathrm{~s}\right)$. Recent work (Marchetti, private communication) has established that the early measurement was due to an artifact, and that the $267 \mathrm{~cm}^{-1}$ is much broader, consistent with our findings.

${ }^{93}$ F. Y. Wu and S. Ezekiel, Laser Focus, March 1977, p. 78.

${ }^{94} \mathrm{R}$. Shoemaker, in Laser and Coherence Spectroscopy, edited by J. Steinfeld (Plenum, New York, 1978), p. 197. 